Soma de Variáveis Aleatórias Equicorrelacionadas e Aplicações em Análise de Risco e Séries Temporais Discretas

Delhi Teresa Paiva Salinas

TESE APRESENTADA

AO INSTITUTO DE MATEMÁTICA E ESTATÍSTICA

DA UNIVERSIDADE DE SÃO PAULO

PARA OBTENÇÃO DO GRAU DE

DOUTOR EM ESTATÍSTICA

Área de Concentração: Probabilidade e Estatística Aplicada Orientador: Prof. Dr. Nikolai Kolev 


\section{SOMA DE VARIÁVEIS ALEATÓRIAS EQUICORRELACIONADAS E APLICACÕES EM ANÁLISE DE RISCO E SÉRIES TEMPORAIS DISCRETAS.}

Este exemplar corresponde à redação final da tese devidamente corrigida e defendida por Delhi Teresa Paiva Salinas e aprovada pela comissão julgadora.

São Paulo, 11 de setembro de 2003.

Banca Examinadora:

- Prof. Dr. Nikolai Kolev (Presidente) - IME - USP

- Prof. Dr. Luis Renato Fontes - IME - USP

- Prof. Dr. Cristiano Augusto Coelho Fernandez - PUC - RJ

- Prof. Dr. Hélio dos Santos Migon - UFRJ

- Prof. Dr. Renato Martins Assunção - UFMG 
A minha família, meus pais Luis e Vilma, meus irmãos Carmen e Lucho, por todo o amor, compreensão, apoio e estímulo. 


\section{Agradecimentos}

Primeiramente, agradeço a. Deus por ter permitido que eu sentisse a sua presença em cada desafio empreendido.

Gostaria de expressar o meu mais profundo agradecimento à minha família, pelo exemplo, apoio e amor. Pela força transmitida e por ter acreditado em minha capacidade, muito obrigada.

Ao orientador desta tese Professor Nikolai Kolev, pelo apoio e disposição para resolver as minhas dúvidas. Agradeço também aos professores membros da banca, por suas sugestões e críticas que muito contribuíram para a versão final deste trabalho.

Meu agradecimento à família Corazza Sef, pelo carinho e apoio, por fazer sentir-me em família, em especial ao meu grande amigo, Henry, por cada um dos momentos em que me brindou com a sua amizade, apoio e carinho. Obrigada por estar sempre presente.

À Suzi, Luciana e Silvia, por brindar-me com uma amizade verdadeira. Ao César, Rosane e Adriano, pelo apoio e imensa solidariedade tão fundamentais neste período. À Kátia, com quem apesar da distância sempre pude contar. Aos demais amigos que de alguma forma ou outra participaram desta etapa de minha vida. A todos vocês, meu eterno agradecimento.

Finalmente, ao CNPq, pelo auxílio financeiro que possibilitou a execução desta tese. 


\section{Resumo}

O interesse básico das companhias de seguro é analisar os riscos acumulados. A teoria clássica assume independência entre os sinistros, mas na prática eles exibem uma estrutura de dependência. Nesta tese, estudaremos somas aleatórias, relaxando a suposição de independência entre as variáveis aleatórias envolvidas. Para quantificar o grau de dependência, usaremos o coeficiente de correlação. Derivaremos expressões explícitas para a função geradora de probabilidade e obteremos a distribuição da soma aleatória no caso em que as variáveis aleatórias são equicorrelacionadas. Além disso, investigaremos mudanças entre os prêmios no caso de riscos dependentes e independentes.

Introduziremos o processo autoregressivo de ordem 1 de valores inteiros correlacionados e sugeriremos duas extensões do processo autoregressivo de ordem 1 discreto.

Finalmente, assumiremos que as variáveis estão em clusters independentes mas que dentro de cada cluster elas são igualmente correlacionadas. Utilizaremos a distribuição multinomial para modelar esta situação. 


\section{Abstract}

The basic interest of the insurance companies is to analize the accumulated risks. The classic theory assumes independence between the claims, but in practice, they exhibit a mutual dependence. In this thesis, we study random sums relaxing the classical assumption of mutual independence between the variables involved. To quantify the degree of the dependence of the accumulated risks we use the coefficient of correlation. Explicit expressions for the joint probability generating function are derived. As particular case the distribution of the dependent random sums are obtained. We investigate changes between premiums in the case of dependent and independent risks.

We introduce the correlated integer valued autoregressive process of order 1 and we suggest two extensions of the discrete autoregressive process of order 1 .

Finally, we assume that the variables are assigned to independent clusters with equal correlation between them within the cluster. The multinomial distribution is an appropriate to model such a situation. 


\section{Sumário}

1 Introdução 1

2 Soma aleatória de variáveis equicorrelacionadas $\quad 6$

2.1 Modelo clássico de risco coletivo . . . . . . . . . . . . 7

2.2 Relação entre variáveis equicorrelacionadas e permutabilidade . . . 8

2.3 Soma de variáveis aleatórias igualmente correlacionadas . . . . . . . 11

2.4 Distribuição binomial correlacionada . . . . . . . . . . . . . . 18

2.5 Uma aplicação em resseguro do tipo stop-loss . . . . . . . . . . 21

3 Correlação entre riscos dependentes 26

3.1 Motivação . . . . . . . . . . . . . . . . . . . . . . . . . 27

3.2 Correlação entre somas dependentes . . . . . . . . . . . . . . . . . . 29

3.3 Modelos de super-dispersão . . . . . . . . . . . . . . . . . . . . 31

3.4 Classificação de riscos dependentes . . . . . . . . . . . . . . . . . . 34

4 Processos INAR(1) e DAR(1) estendidos 38

4.1 Processo INAR(1) e sua versão correlacionada . . . . . . . . . . . 39 
4.2 Duas extensões do processo DAR(1) . . . . . . . . . . . . 58

5 Modelo multinomial latente para somas aleatórias

5.1 Modelo multinomial correlacionado . . . . . . . . . . . . . . . 71

5.2 Estimação dos parâmetros e simulação . . . . . . . . . . 76

6 Conclusões e trabalhos futuros $\quad 81$

A Derivadas do capítulo $4 \quad 83$

$\begin{array}{ll}\text { B Programas do capítulo 4 } & 87\end{array}$

$\begin{array}{ll}\text { C Derivadas do capítulo } 5 & 97\end{array}$

$\begin{array}{lr}\text { D Programas do capítulo 5 } & 100\end{array}$

$\begin{array}{ll}\text { Referências Bibliográficas } & \mathbf{1 1 5}\end{array}$ 


\section{Capítulo 1}

\section{Introdução}

O objetivo básico do modelo clássico de risco é avaliar somas aleatórias que representam somas acumuladas de sinistros em algum período de tempo fixo.

A maioria dos estudos feitos até agora assumiu independência entre as variáveis aleatórias envolvidas (ver e.g., Bowers et al., 1997). Durante os últimos cinco anos foram publicados mais de 100 artigos nos quais se encontram estudos sobre somas aleatórias com alguma estrutura de dependência entre as variáveis aleatórias (ver Kass et al., 2001).

Muitos autores têm estudado os modelos com diferentes tipos de dependência entre os sinistros. Por exemplo, Gerber (1982) estudou o problema de ruína quando os sinistros consecutivos formam um modelo autoregressivo; Asmussen (1989) usou o modelo modulado Markoviano que exibe um tipo especial de dependência.

A pesquisa sobre o tipo e grau de dependência entre os sinistros tem sido um tópico central na área atuarial. Veja, por exemplo Dhaene et al. (2000), Goovaerts et al. (2000), Embrechts et al. (2002), Frey e McNeil (2001), Frey et al. (2001), Kaas et al. (2000), Simon et al. (2000), Vyncke et al. (2001). A seguir, 
apresentamos um resumo de alguns artigos importantes.

Dhaene e Goovaerts (1996) estudaram a distribuição de funções dos riscos individuais. Eles investigaram como a mudança da suposição de dependência influencia os prêmios do tipo "stop-loss" de carteiras com dependência par a par. Parte desses resultados foram generalizados em Dhaene e Goovaerts (1997), que consideraram o modelo de vida individual, i.e., o caso onde cada risco individual tem uma distribuição concentrada em dois pontos, em zero e em algum valor positivo.

Goovaerts e Dhaene (1996) mostraram que a aproximação da Poisson composta ainda é bem comportada sob certas circunstâncias quando é relaxada a suposição de independência entre os riscos individuais.

Wang e Dhaene (1998) investigaram a noção de dependência entre riscos e seus efeitos nos prêmios do tipo "stop-loss". Eles discutiram em detalhe o conceito de comonotonicidade, que é um caso extremo de dependência. Mostraram também as distribuições dos riscos individuais no caso bivariado. Da mesma maneira, este tipo de prêmio foi de interesse de muitos pesquisadores. Veja Alberts (1999), por exemplo.

Dhaene e Denuit (1999) estudaram a dependência nos espaços de Fréchet contendo riscos mutuamente exclusivos. Eles mostraram que, sob algumas suposições razoáveis, a estrutura de dependência no sentido de prêmios do tipo "stop-loss" mínimo para os sinistros agregados envolvidos é obtida com o limite inferior de Fréchet, que corresponde precisamente a riscos mutuamente exclusivos do espaço de Fréchet.

Goovaerts, Dhaene e Schepper (2000) apresentaram uma aproximação fácil para a função de distribuição do valor presente de uma seqüência cash flows, que é o limite superior para a função de distribuição original. 
Ambagaspitiya (1998) discutiu a distribuição de perda agregada para uma carteira de negócios, que pode ser expressa como a união de classes disjuntas de negócios. Ela assumiu que as perdas para cada classe de negócios pode ser apresentada em termos de variáveis aleatórias compostas que são correlacionadas. Ambagaspitiya (1999) apresentou duas classes de distribuição de sinistros agregados correlacionados, considerando o número de sinistros, variáveis aleatórias univariadas, o tamanho dos sinistros e variáveis aleatórias multivariadas.

Hu e Wu (1999) generalizaram alguns dos resultados de Dhaene e Goovaerts (1996, 1997). Primeiro consideraram o tipo de dependência negativa entre indivíduos numa carteira que dá origem aos sinistros agregados mais seguros. Eles também aplicaram a dependência super-aditiva que é uma alternativa para comparar as carteiras de sinistros em risco com estruturas de dependência multivariada.

Dhaene et al. (2002a) apresentaram resultados novos para aproximações de somas aleatórias quando as distribuições marginais são conhecidas, mas a distribuição conjunta (estrutura estocástica de dependência) é desconhecida ou muito complicada. Eles mostraram que a soma de elementos do vetor aleatório $\left(Y_{1}, \ldots, Y_{n}\right)$ tem limite superior quando a distribuição conjunta do vetor aleatório é comonotônica. Isso significa que todos os pares das possíveis realizações do vetor estão ordenados para qualquer par de elementos. No artigo seguinte, Dhaene et al. (2002b) apresentaram diferentes aplicações do conceito de comonotonicidade na área financeira e atuarial.

Devido à importância e evolução da pesquisa de somas aleatórias na área atuarial, o objetivo deste trabalho é estudar somas aleatórias de variáveis aleatórias que são dependentes. Além disso, serão apresentadas algumas aplicações em séries temporais em tempo discreto. Ao longo do trabalho, a dependência entre as variáveis será medida por meio do coeficiente de correlação linear de Pearson. 
Esta tese está dividida em cinco capítulos. No capítulo 2, apresentaremos alguns resultados de variáveis aleatórias com a mesma estrutura de correlação (equicorrelação). Além disso, mostraremos a distribuição de variáveis aleatórias Bernoulli igualmente correlacionadas. Este estudo será feito por meio da função geradora de probabilidade, donde se obterá o resultado básico da soma aleatória de variáveis aleatórias equicorrelacionadas. Apresentaremos também a relação de equicorrelação com permutabilidade e finalmente uma aplicação.

No capítulo 3, apresentaremos a correlação entre riscos dependentes. Para isso, consideraremos as dependências entre os sinistros e também entre o número de sinistros de cada companhia. Investigaremos mudanças no prêmio que surgem da interdependência de linhas de seguro. Além disso, introduziremos o prêmio de tipo "stop-loss", que será ilustrado com um exemplo.

No capítulo 4, mostraremos os processos INAR(1) e DAR(1) estendidos. O primeiro será uma extensão do modelo estudado por Al-Osh e Alzaid (1987) e McKenzie (1988) considerando respostas binárias igualmente correlacionadas. O segundo será uma extensão do processo $\operatorname{DAR}(1)$, introduzido por Jacobs e Lewis (1978). Estas duas extensões estarão baseadas em operadores definidos no capítulo 2. A idéia é obter modelos mais flexíveis quando temos processos de contagem. Ambos os processos serão ilustrados com exemplos.

No capítulo 5, consideraremos que as variáveis são distribuídas em clusters independentes e assumiremos que existe dependência só entre variáveis que pertencem ao mesmo cluster. Para modelar esta situação, utilizaremos a distribuição multinomial. Além disso, obteremos as estimativas dos parâmetros de quatro modelos usando o método de máxima verossimilhança. As ilustrações correspondentes também serão mostradas.

No capítulo 6, apresentaremos as conclusões finais deste trabalho bem como 
algumas sugestões para pesquisas futuras.

Finalmente, nos Apêndices A e C, serão apresentadas as derivadas parciais dos modelos CINAR(1) e DAR(1) estendidos apresentados no capítulo 4. Nos Apêndices B e D encontram-se os programas realizados na linguagem de programação Ox (veja Doornik, 1996), os quais serão usados para calcular as estimativas da cada um dos modelos. 


\section{Capítulo 2}

\section{Soma aleatória de variáveis}

\section{equicorrelacionadas}

Neste capítulo, estudaremos somas aleatórias de variáveis aleatórias que são dependentes. Mediremos essa dependência por meio do coeficiente de correlação. Na seção 2.1, interpretaremos a soma aleatória em termos do modelo clássico de risco coletivo. De fato, os sinistros são variáveis aleatórias, que assumiremos serem equicorrelacionadas. Na seção 2.2 , apresentaremos uma relação entre variáveis igualmente correlacionadas e o conceito de permutabilidade. O resultado básico será obtido na seção 2.3 (a função geradora de probabilidade da soma aleatória de variáveis aleatórias equicorrelacionadas). Na seção 2.4, mostraremos o modelo Binomial correlacionado como um caso particular e na seção 2.5, apresentaremos uma aplicação em resseguro do tipo "stop-loss". 


\subsection{Modelo clássico de risco coletivo}

Consideremos um contrato de seguro coletivo em algum período de tempo fixo $(0, T]$, em que $T$ usualmente é um ano. Denotemos por $N$ o número de sinistros neste período e $Y_{1}, Y_{2}, \ldots, Y_{N}$ os correspondentes sinistros observados (que são variáveis aleatórias). A Figura 2.1 representa o processo de risco sendo que $u$ é o capital inicial e $c>0$ é o índice de lucro, $t \in[0, T]$.

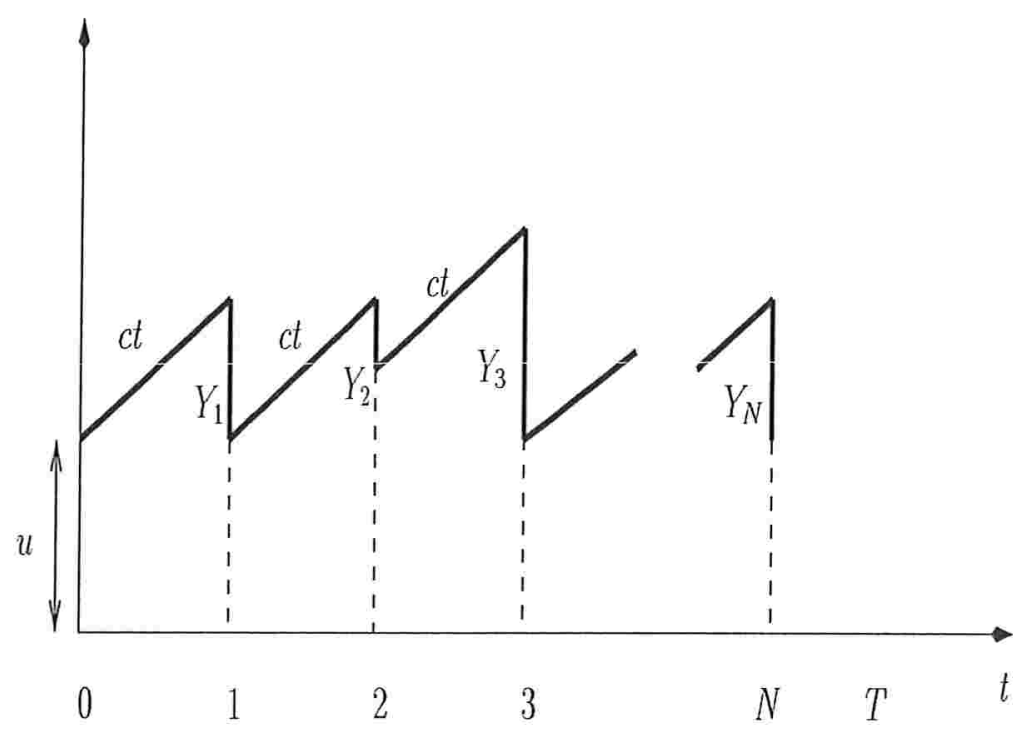

Figura 2.1. Processo de Risco.

O objetivo principal do modelo de risco coletivo é avaliar a soma aleatória

$$
S_{N} \stackrel{d}{=} \sum_{i=1}^{N} Y_{i}
$$

que é a soma acumulada dos sinistros no período considerado. A suposição básica na teoria clássica é que as variáveis envolvidas sejam independentes; veja Kaas et al. (2001). Ou seja: 
(i) $N$ e $\left(Y_{1}, Y_{2}, \ldots\right)$ são variáveis aleatórias (v.a.'s) independentes;

(ii) $Y_{1}, Y_{2}, \ldots$ são independentes e

(iii) $Y_{1}, Y_{2}, \ldots$ têm distribuição comum.

A suposição de independência mútua, i.e., as condições (i) e (ii) são muito convenientes porque tornam a matemática muito fácil. Por exemplo, os algoritmos implementados, tais como a recursão de Panjer, a recursão de De Pril, a convolução e a aproximação de momentos estão baseados na suposição de independência mútua; veja Bowers et al. (1997).

Na prática, os sinistros (riscos) são dependentes porque eles contêm usualmente um fator aleatório comum ou estão influenciados pelo mesmo ambiente econômico ou geográfico. Na presença de dependência entre os sinistros, a lei dos grandes números implica que o risco médio fica cada vez mais imprevisível. A suposição de independência pode ser usada como uma primeira aproximação da distribuição verdadeira da soma acumulada.

Nosso objetivo será avaliar a soma aleatória (2.1) quando os sinistros $Y_{1}, Y_{2}, \ldots$ são dependentes. Quantificaremos esta dependência através do coeficiente de correlação e estudaremos o efeito desta correlação na soma aleatória definida em (2.1).

\subsection{Relação entre variáveis equicorrelacionadas e permutabilidade}

Sem perda de generalidade (e por simplicidade), consideremos uma seqüência $\left\{Y_{i}\right\}_{i=1}^{n}$ finita de v.a.'s não negativas que assumem valores inteiros e que são iden- 
ticamente distribuídas

$$
\mathbb{P}(Y=r)=\pi_{r}, \quad r=0,1,2, \ldots, k, \quad \sum_{r=0}^{k} \pi_{r}=1,
$$

com os dois primeiros momentos finitos. Denotamos a Função Geradora de Probabilidade (FGP) da v.a. $Y$ por $G_{Y}(t)=\mathbb{E}\left(t^{Y}\right)$. Assumamos que $Y_{i}^{\prime}$ 's são igualmente correlacionados, i.e.,

$$
\operatorname{Corr}\left(Y_{i}, Y_{j}\right)=\rho \in[0,1], \quad i \neq j, \quad i, j=1,2, \ldots, n
$$

Em conseqüência, os sinistros $Y_{i}^{\prime}$ 's não são mais independentes e sim equicorrelacionados, i.e., relaxamos a condição (ii) da seção 2.1. Então todo par de elementos do vetor aleatório $\left(Y_{1}, \ldots, Y_{n}\right)$ é equicorrelacionado. Isso significa que todos os elementos fora da diagonal da matriz de correlação são iguais a $\rho$.

Em seguida, apresentaremos uma relação entre variáveis aleatórias equicorrelacionadas e o conceito de permutabilidade.

Uma classe de v.a.'s dependentes são as seqüências permutáveis de seqüências finitas. Dizemos que as v.a.'s $Y_{1}, Y_{2}, \ldots, Y_{n}$ são permutáveis se a distribuição do vetor $\left(Y_{i_{1}}, Y_{i_{2}}, \ldots, Y_{i_{n}}\right)$ é idêntica à distribuição do vetor $\left(Y_{1}, Y_{2}, \ldots, Y_{n}\right)$ para todas as permutações $\left(i_{1}, i_{2}, \ldots, i_{n}\right)$ de índices $(1,2, \ldots, n)$.

Note que, se as v.a.'s $Y_{1}, Y_{2}, \ldots, Y_{n}$ são independentes e identicamente distribuídas (i.i.d.), então elas são permutáveis. Em conseqüência, o conceito de permutabilidade inclui como caso particular o conceito de independência.

Nosso modelo é fortemente conectado ao conceito de permutabilidade que restringe a possível estrutura de correlação para as seqüências de v.a.'s, com primeiro e segundo momentos finitos. Para ver isto, padronizemos os $Y_{i}^{\prime}$ 's, i.e., $X_{i}=\frac{Y_{i}-\boldsymbol{E}\left(Y_{i}\right)}{\sqrt{\operatorname{Var}\left(Y_{i}\right)}}$. Assim, teremos $\mathbb{E}\left(X_{i}\right)=0$ e $\operatorname{Var}\left(X_{i}\right)=1$, para $i=1,2, \ldots, n$. Se $\left(Y_{1}, Y_{2}, \ldots, Y_{n}\right)$ 
é uma seqüência de v.a.'s permutáveis, então existe a correlação

$$
\rho=\operatorname{Corr}\left(Y_{i}, Y_{j}\right)=\operatorname{Cov}\left(X_{i}, X_{j}\right)=\mathbb{E}\left(X_{i} X_{j}\right), \quad i \neq j .
$$

Consideremos a desigualdade

$$
0 \leq \mathbb{E}\left(\sum_{i=1}^{n} X_{i}\right)^{2}=\sum_{i=1}^{n} \mathbb{E} X_{i}^{2}+\sum_{i \neq j} \mathbb{E}\left(X_{i} X_{j}\right)=n+n(n-1) \rho .
$$

Assim,

$$
\rho \geq-\frac{1}{n-1}, \quad \text { com igualdade se e somente se } \quad \sum_{i=1}^{n} X_{i}=0, \quad \text { q.c. }
$$

Inversamente, cada $\rho$ satisfazendo (2.4) ocorre como a correlação em alguma seqüência permutável. Para mostrar isto, consideremos uma seqüência $\left(R_{1}, R_{2}, \ldots, R_{n}\right)$ de v.a.'s i.i.d. com $\mathbb{E}\left(R_{i}\right)=0$ e $\operatorname{Var}\left(R_{i}\right)=1$ e definamos

$$
X_{i}=R_{i}+d \sum_{i=1}^{n} R_{i}, \quad i=1,2, \ldots, n,
$$

para alguma constante $d$. Então $\left(X_{1}, X_{2}, \ldots, X_{n}\right)$ é uma seqüência permutável, e

$$
\rho=\mathbb{E}\left(X_{i} X_{j}\right)=1-\frac{1}{n d^{2}+2 d+1}, \quad i \neq j .
$$

Quando $d$ varia, $d \in\left[-\frac{1}{n}, \infty\right]$, encontramos todos os valores de $\rho \in\left[-\frac{1}{n-1}, 1\right]$. Em particular, $\rho=-\frac{1}{n-1}$ para $d=-\frac{1}{n}$ (para uma amostragem sem reposição de uma urna de $n$ elementos, ou se as v.a.'s $X_{i}$ têm distribuição multinomial simétrica); e $\rho=1$ considerando $X_{1}=X_{2}=\ldots=X_{n}$ q.c. (i.e., quando $d \rightarrow \infty$ ).

Note que o determinante da matriz de covariância de $\left(X_{1}, X_{2}, \ldots, X_{n}\right)$ é igual a $(1-\rho)^{n-1}[1-\rho(n-1)]$. Ele deve ser não negativo, i.e., $\rho \geq-(n-1)^{-1}$, o que confirma a relação (2.4).

No caso infinito (quando $n \rightarrow \infty),(2.4)$ implica $\rho \geq 0$. 


\subsection{Soma de variáveis aleatórias igualmente cor- relacionadas}

Um caso especial e simples é das seqüências binárias permutáveis de valores 0 e 1. Uma seqüência finita de eventos é chamada permutável se suas variáveis indicadoras são permutáveis.

Seja $Z_{1}, Z_{2}, \ldots, Z_{n}$ uma seqüência de v.a.'s Bernoulli permutáveis com $\mathbb{P}\left(Z_{i}=1 \mid p\right)=p$, para $i=1,2, \ldots, n$. Definamos

$$
w_{0}=1 \quad \text { e } \quad w_{k}=\mathbb{P}\left(Z_{1}=1, Z_{2}=1, \ldots, Z_{k}=1\right), \quad k=1,2, \ldots, n .
$$

Seja $\xi$ uma v.a. com valores em $[0,1]$. Assim, pelo teorema de DeFinetti, temos (veja DeFinetti, 1975):

$$
\mathbb{P}\left(Z_{1}=1, Z_{2}=1, \ldots, Z_{k}=1, Z_{k+1}=0, \ldots, Z_{n}=0\right)=\int_{0}^{1} p^{k}(1-p)^{n-k} d \xi(p) .
$$

Portanto, o número $w_{n}$ pode ser escrito da seguinte forma

$$
w_{n}=\mathbb{P}\left(Z_{1}=1, Z_{2}=1, \ldots, Z_{n}=1\right)=\int_{0}^{1} p^{n} d \xi(p) .
$$

Então a seqüência decrescente $1=w_{0} \geq w_{1} \geq \ldots \geq w_{n}>0$ representa os momentos de alguma distribuição de probabilidade $\xi(p)$ definida em $[0,1]$.

A partir desta última equação podemos encontrar a distribuição da soma de v.a.'s Bernoulli permutáveis $S_{n}=\sum_{i=1}^{n} Z_{i}$.

Por outro lado, observemos que a probabilidade de "sucesso" $p$ e a covariância podem ser calculadas por $w_{1}$ e $w_{2}$ da seguinte maneira:

$$
\begin{aligned}
w_{1} & =\mathbb{P}\left(Z_{1}=1\right)=\mathbb{P}\left(Z_{i}=1\right) \\
w_{2}-w_{1}^{2} & =\mathbb{P}\left(Z_{1}=1, Z_{2}=1\right)-w_{1}^{2}=\mathbb{E}\left(Z_{1} Z_{2}\right)-\mathbb{E}\left(Z_{1}\right) \mathbb{E}\left(Z_{2}\right)=\operatorname{Cov}\left(Z_{1}, Z_{2}\right) .
\end{aligned}
$$


Seja $a_{n, k}$ a probabilidade de qualquer seqüência de $n$ variáveis Bernoulli permutáveis que contenha $k \leq n$ "sucessos". Então, a distribuição da seqüência $\left\{Z_{n}\right\}$ é completamente determinada pelos números $a_{n, k}=\mathbb{P}\left(Z_{1}=i_{1}, Z_{2}=i_{2}, \ldots, Z_{n}=i_{n} \mid \sum_{j=1}^{n} Z_{j}=k\right)=\sum_{j=0}^{n-k}\left(\begin{array}{c}n-k \\ j\end{array}\right)(-1)^{j} w_{k+j}$ se $\sum_{j=1}^{n} i_{j}=k$; veja Madsen (1993).

Portanto, a seqüência $\left\{w_{n}\right\}$ pode ser usada para gerar a distribuição conjunta das v.a.'s Bernoulli permutáveis.

Agora calculemos a distribuição de $S_{n}$, i.e., do número total de sucessos em $n$ eventos para a distribuição binomial generalizada. Ou seja,

$$
\mathbb{P}\left(S_{n}=k\right)=\left(\begin{array}{l}
n \\
k
\end{array}\right) a_{n, k} .
$$

Várias famílias de distribuições podem ser geradas expressando a seqüência $1=w_{0} \geq w_{1} \geq \ldots \geq w_{n} \geq 0$ em forma paramétrica. Existem dois casos particulares cuja seqüência permutável pode ser considerada "degenerada".

Primeiro, se $w_{k}=p^{k}$, com $p \in[0,1]$ e $k=1,2, \ldots, n$, o que significa que $\xi(p)=1$ para algum valor particular $p \in(0,1)$. Isto corresponde ao caso de independência (o coeficiente de correlação é igual a zero). Neste caso, pode ser visto que $a_{n, k}=p^{k}(1-p)^{n-k}$, para $k=0,1, \ldots, n$.

Segundo, consideremos a seqüência definida por $w_{0}=1$ e $w_{k}=p$, para $k=$ $1,2, \ldots, n$. Esta seqüência corresponde ao caso de dependência total (o coeficiente de correlação é igual a um), i.e., $a_{n, n}=p$ e $a_{n, 0}=1-p$. Isto significa que em $n$ eventos existem $n$ sucessos com probabilidade $p$ e 0 sucessos com probabilidade $(1-p)$.

Madsen (1993) estabelece que se as seqüencias de probabilidades $\left\{w_{1 k}\right\}$ e $\left\{w_{2 k}\right\}$ 
possuírem primeiros termos iguais a 1, i.e., $w_{10}=w_{20}=1$, e suas diferenças forem positivas, então $w_{k}=\phi w_{1 k}+(1-\phi) w_{2 k}$, com $\phi \in[0,1]$, para $k=1,2, \ldots, n$ também satisfaz essas condições. Assim,

$$
\delta^{k} w_{n-k} \geq 0, \quad 0 \leq k \leq n \quad \text { e } \quad \sum_{k=0}^{n}\left(\begin{array}{l}
n \\
k
\end{array}\right) \delta^{k} w_{n-k}=1,
$$

em que $\delta^{k} w_{k}$ é definido recursivamente por

$$
\delta^{0} w_{k}=w_{k}, \quad \delta w_{k}=w_{k}-w_{k+1} \quad \text { e } \quad \delta^{k} w_{k}=\delta\left(\delta^{k-1} w_{k}\right), \quad \text { para } \quad k \geq 1 .
$$

Esta distribuição de probabilidade correspondería à mistura de distribuições usada em (2.5) para gerar a distribuição conjunta de v.a.'s Bernoulli permutáveis. A construção (2.5) implica unicamente dependência positiva. Por outro lado, quando o coeficiente de correlação é negativo, as v.a.'s correspondentes nunca podem ser condicionalmente independentes e identicamente distribuídas.

Aplicando as considerações acima, geramos uma família de distribuição de dois parâmetros. Combinando os dois casos dados anteriormente, temos

$$
\begin{aligned}
& w_{0}=1 \mathrm{e} \\
& w_{k}=\phi p+(1-\phi) p^{k} \quad \text { para } \quad k=1,2, \ldots, n .
\end{aligned}
$$

Para este último modelo, o parâmetro $\phi$ é simplesmente a correlação par a par entre as variáveis Bernoulli. Para visualizar isto, consideremos a correlação entre $Z_{1}$ e $Z_{2}$. Note que

$$
\begin{aligned}
\operatorname{Cov}\left(Z_{1}, Z_{2}\right) & =\mathbb{E}\left(Z_{1} Z_{2}\right)-\mathbb{E}\left(Z_{1}\right) \mathbb{E}\left(Z_{2}\right)=w_{2}-w_{1}{ }^{2} \\
& =\phi p+(1-\phi) p^{2}=\phi p-\phi p^{2}=\phi p(1-p) .
\end{aligned}
$$

Por outro lado, como a variância de cada $Z_{i}$ é $p(1-p)$, então a correlação é dada por

$$
\rho=\operatorname{Corr}\left(Z_{1}, Z_{2}\right)=\phi
$$


Observe que quando $\phi=0,(2.6)$ se reduz ao caso de independência e quando $\phi=1$, ao caso de dependência completa.

Nosso objetivo é estudar a FGP conjunta da distribuição conjunta do vetor $\left(Z_{1}, \ldots, Z_{n}\right)$ gerada pelo modelo $(2.6)$.

Sejam $Y_{i}$ 's v.a.'s identicamente distribuídas, com distribuição dada por (2.2). A FGP conjunta do vetor aleatório $\mathrm{Y}=\left(Y_{1}, Y_{2}, \ldots, Y_{n}\right)$, sendo $\mathrm{t}=\left(t_{1}, t_{2}, \ldots, t_{n}\right)$ é definida por

$$
G_{\mathrm{Y}}(\mathrm{t})=\sum_{r_{1}=0}^{k} \ldots \sum_{r_{n}=0}^{k} t_{1}^{Y_{1}} \ldots t_{n}^{Y_{n}} \mathbb{P}\left(Y_{1}=r_{1}, \ldots, Y_{n}=r_{n}\right), \quad\left|t_{i}\right| \leq 1 .
$$

Consideremos duas seqüências de v.a's Bernoulli $Z_{1}^{I}, \ldots, Z_{n}^{I}$ e $Z_{1}^{D}, \ldots, Z_{n}^{D}$ com distribuição comum, i.e., $\mathbb{P}\left(Z_{i}^{I}=1\right)=p=\mathbb{P}\left(Z_{i}^{D}=1\right), i=1, \ldots, n$.

$\operatorname{Sejam}\left(Z_{1}^{I}, \ldots, Z_{n}^{I}\right)$ as componentes independentes do vetor $\mathbf{Z}^{I}=\left(Z_{1}^{I}, \ldots, Z_{n}^{I}\right)$. A FGP do vetor $\mathbf{Z}^{I}$ é dada por

$$
G_{Z^{I}}(\mathrm{t})=\prod_{i=1}^{n} G_{Z_{i}^{I}}\left(t_{i}\right)
$$

$\operatorname{com} G_{Z_{i}^{I}}\left(t_{i}\right)=1-p+p t_{i}$.

Sejam $Z_{1}^{D}, \ldots, Z_{n}^{D}$ as componentes que possuem dependência total. Suponhemos que o vetor correspondente $\mathbf{Z}^{D}=\left(Z_{1}^{D}, \ldots, Z_{n}^{D}\right)$ é independente do vetor $\mathbf{Z}^{I}$. $\mathrm{O}$ vetor $\mathbf{Z}^{D}$ tem $\mathrm{FGP}$ dada por

$$
G_{\mathrm{Z}^{D}}(\mathbf{t})=1-p+p\left(t_{1} t_{2} \ldots t_{n}\right)
$$

Consideremos o vetor $\mathbf{Z} \stackrel{d}{=} \rho \mathbf{Z}^{D}+(1-\rho) \mathbf{Z}^{I}$, com $\rho \in[0,1]$. O vetor $\mathbf{Z}$ tem FGP dada por

$$
G_{\mathbf{Z}}(\mathrm{t})=\rho\left[1-p+p\left(t_{1} t_{2} \ldots t_{n}\right)\right]+(1-\rho) \prod_{i=1}^{n} G_{Z_{i}^{I}}\left(t_{i}\right)
$$


É facil ver que os elementos do vetor $\mathbf{Z}=\left(Z_{1}, \ldots, Z_{n}\right)$, são equicorrelacionados (veja (2.6)). A relação (2.7) é uma mistura de distribuições de v.a.'s Bernoulli com dependência perfeita (o vetor $\mathbf{Z}^{D}$ ) e independência completa (o vetor $\mathbf{Z}^{I}$ ).

Sejam $Y_{i}^{\prime}$ 's v.a.'s identicamente distribuídas com distribuição dada por (2.2). Sejam $Z_{i}=1\left\{Y_{i}>M\right\}, i=1,2, \ldots, n$ em que $1\{\cdot\}$ é a função indicadora e $M>0$, fixo. A mistura de distribuições (2.7) estende-se unicamente para a FGP do vetor $\mathrm{Y}$ da seguinte maneira:

$$
G_{\mathbf{Y}}(\mathrm{t})=\rho\left[\sum_{r=0}^{k} \pi_{r}\left(\prod_{i=1}^{n} t_{i}\right)^{r}\right]+(1-\rho) \prod_{i=1}^{n} G_{Y_{i}}\left(t_{i}\right), \quad\left|t_{i}\right| \leq 1 .
$$

A última expressão foi primeramente apresentada por Tallis (1962).

A distribuição marginal de $Y_{j}$ é obtida imediatamente de (2.8), substituindo $t_{i}=1$ para $i \neq j, i, j=1,2, \ldots, n$. De (2.8), verificamos que

$$
\operatorname{Cov}\left(Y_{i}, Y_{j}\right)=\mathbb{E}\left(Y_{i} Y_{j}\right)-\mathbb{E}^{2}(Y)=\rho \operatorname{Var}(Y)
$$

sendo $\mathbb{E}(Y)=\sum_{r=0}^{k} r \pi_{r}$ e $\operatorname{Var}(Y)=\sum_{r=0}^{k} r^{2} \pi_{r}-\mathbb{E}^{2}(Y)$.

Isso confirma o fato de que o parâmetro $\rho$ em (2.8) é justamente o coeficiente de correlação entre $Y_{i}$ e $Y_{j}$ para $i \neq j$ (veja (2.3)). Dependendo das probabilidades (2.2), o valor de $\rho$ em (2.8) pode ser negativo, i.e., $\rho \in\left[-\frac{1}{n-1}, 0\right]$ (veja (2.4)). Um exemplo particular (quando $k=1$ ) será dado depois da equação (2.19).

A relação (2.8) especifica a distribuição conjunta do vetor aleatório Y. Quando $\rho=0$, a distribuição converte-se no produto de $n$ variáveis idênticas, o que mostra que as variáveis são independentemente distribuídas. Por outro lado, quando $\rho=1$, a distribuição se "degenera" de tal forma que

$$
\mathbb{P}\left(Y_{1}=r, Y_{2}=r, \ldots, Y_{n}=r\right)=\pi_{r} \quad \text { para } \quad r=0,1, \ldots, k
$$


e todas as outras probabilidades conjuntas são zero. Portanto, (2.8) é a média ponderada da distribuição com correlação perfeita e com independência completa, com os pesos $\rho$ e $1-\rho$, respectivamente.

Consideremos agora uma nova variável $S_{n}=Y_{1}+\cdots+Y_{n}$. De (2.8), obtemos a FGP de $S_{n}$ substituindo $t_{j}$ por $t$, para $j=1,2, \ldots, n$;

$$
G_{S_{n}}(t)=\mathbb{E}\left(t^{S_{n}}\right)=\rho\left(\sum_{r=0}^{k} \pi_{r} t^{n r}\right)+(1-\rho)\left(G_{Y}(t)\right)^{n},
$$

cuja esperança e variância são obtidas da seguinte forma. Por definição, temos que

$$
\left.G_{S_{n}}^{(v)}(t)\right|_{t=1}=\mathbb{E}\left[S_{n}\left(S_{n}-1\right)\left(S_{n}-2\right) \ldots\left(S_{n}-v+1\right)\right]
$$

No nosso caso, para $v=1$ e $v=2$, obtemos

$$
\begin{aligned}
G_{S_{n}}^{\prime}(t) & =\rho n \sum_{r=0}^{k} r \pi_{r} t^{n r-1}+(1-\rho) n\left(G_{Y}(t)\right)^{n-1} G_{Y}^{\prime}(t) \\
\left.G_{S_{n}}^{\prime}(t)\right|_{t=1} & =\rho n \sum_{r=0}^{k} r \pi_{r}+(1-\rho) n\left(G_{Y}(1)\right)^{n-1} G_{Y}^{\prime}(1) \\
& =\rho n \mathbb{E}(Y)+(1-\rho) n \mathbb{E}(Y) .
\end{aligned}
$$

Portanto, a esperança de $S_{n}$ é dada por

$$
\mathbb{E}\left(S_{n}\right)=n \mathbb{E}(Y)
$$

Sabemos que a variância de $S_{n}$ é dada por

$$
\operatorname{Var}\left(S_{n}\right)=\left.\left\{G_{S_{n}}^{\prime \prime}(t)+G_{S_{n}}^{\prime}(t)-\left[G_{S_{n}}^{\prime}(t)\right]^{2}\right\}\right|_{t=1}
$$

Para o primeiro termo da expressão (2.11), temos: 


$$
\begin{aligned}
G_{S_{n}}^{\prime \prime}(t)= & \rho n \sum_{r=0}^{k} r \pi_{r} t^{n r-2}(n r-1)+(1-\rho) n \times \\
& \times\left[(n-1)\left(G_{Y}(t)\right)^{n-2}\left(G_{Y}^{\prime}(t)\right)^{2}+\left(G_{Y}(t)\right)^{n-1}\left(G_{Y}^{\prime \prime}(t)\right)\right] . \\
\left.G_{S_{n}}^{\prime \prime}(t)\right|_{t=1}= & \rho n^{2} \sum_{r=0}^{k} r^{2} \pi_{r}+(1-\rho) n\left[(n-1)\left(G_{Y}^{\prime}(1)\right)^{2}+\left(G_{Y}^{\prime \prime}(1)\right)\right] \\
= & \rho n^{2} \mathbb{E}\left(Y^{2}\right)-\rho n \mathbb{E}(Y)+(1-\rho) n\left[(n-1) \mathbb{E}^{2}(Y)+\mathbb{E}(Y(Y-1))\right] \\
= & \rho n^{2} \operatorname{Var}(Y)+(1-\rho) n \operatorname{Var}(Y)+n^{2} \mathbb{E}^{2}(Y)-n \mathbb{E}(Y) \\
= & n \operatorname{Var}(Y)[1+(n-1) \rho]+n^{2} \mathbb{E}^{2}(Y)-n \mathbb{E}(Y) .
\end{aligned}
$$

Substituindo em (2.11), obtemos a expressão da variância

$$
\operatorname{Var}\left(S_{n}\right)=n \operatorname{Var}(Y)[1+(n-1) \rho]
$$

Mas $\operatorname{Var}\left(S_{n}\right) \geq 0$, e portanto, $\rho$ pode ser negativo, como já vimos em (2.4).

Nosso interesse é encontrar a FGP de $S_{N} \stackrel{d}{=} \sum_{i=1}^{N} Y_{i}$, sendo $N$ uma v.a. discreta e não negativa, independente das $Y_{i}^{\prime}$ 's, com função de distribuição

$$
\mathbb{P}(N=n)=p_{n}, \quad n=0,1, \ldots, \quad \sum_{n=0}^{\infty} p_{n}=1 .
$$

Seja $G_{N}(t)$ a FGP de $N$. Usando a fórmula de probabilidade total e (2.9), obtemos a FGP de $S_{N}$

$$
\begin{aligned}
G_{S_{N}}(t) & =\mathbb{E}\left(t^{S_{N}}\right)=\mathbb{E}\left[\mathbb{E}\left(t^{Y_{1}+\ldots+Y_{N}} \mid N\right)\right] \\
& =\sum_{n=0}^{\infty} \mathbb{E}\left(t^{Y_{1}+\ldots+Y_{n}}\right) \mathbb{P}(N=n) \\
& =\sum_{n=0}^{\infty}\left[\rho\left(\sum_{r=0}^{k} \pi_{r} t^{n r}\right)+(1-\rho)\left(G_{Y}(t)\right)^{n}\right] p_{n} \\
& =\rho \sum_{r=0}^{k} \pi_{r} G_{N}\left(t^{r}\right)+(1-\rho) G_{N}\left(G_{Y}(t)\right)_{i}
\end{aligned}
$$


ou seja,

$$
G_{S_{N}}(t)=\rho \sum_{r=0}^{k} \pi_{r} G_{N}\left(t^{r}\right)+(1-\rho) G_{N}\left(G_{Y}(t)\right),
$$

com $G_{N}(t)$ e $G_{Y}(t)$ sendo as FGP's de $N$ e dos $Y_{i}$ 's, respectivamente. A relação (2.13) pode ser representada de forma mais geral usando a função geradora de momentos (FGM) quando as v.a.'s $Y_{i}$ 's tiverem distribuição arbitrária (veja Kolev e Paiva (2000)).

Se as v.a.'s $Y_{i}$ 's são independentes, podemos considerar $\rho=0$ em (2.13), o que gera o resultado clássico bem conhecido

$$
G_{S_{N}}(t)=G_{N}\left(G_{Y}(t)\right)
$$

A partir de (2.13), encontramos a esperança e variância de $S_{N}$ :

$$
\begin{aligned}
\mathbb{E}\left(S_{N}\right)= & \mathbb{E}(N) \mathbb{E}(Y), \\
\operatorname{Var}\left(S_{N}\right)= & \mathbb{E}(N) \operatorname{Var}(Y)+\operatorname{Var}(N)[\mathbb{E}(Y)]^{2} \\
& +\rho \operatorname{Var}(Y) \mathbb{E}[N(N-1)] .
\end{aligned}
$$

As expressões (2.14) e (2.15) da esperança e variância da soma acumulada de v.a.'s equicorrelacionadas serão bastante usadas ao longo deste trabalho, pois o prêmio do risco usualmente é uma função da $\mathbb{E}\left(S_{N}\right)$ e da $\operatorname{Var}\left(S_{N}\right)$.

\subsection{Distribuição binomial correlacionada}

Sabemos que a distribuição Binomial é a soma de $n$ v.a's. independentes Bernoulli que assumem valores 0 e 1 . Particularizemos a expressão (2.9) para o caso $k=1$, e assim consideremos as v.a.'s $Z_{i}$ 's identicamente distribuídas Bernoulli com parâmetro $\alpha$, i.e., $\pi_{0}=1-\alpha$ e $\pi_{1}=\alpha$, igualmente correlacionadas, 
$\operatorname{Corr}\left(Z_{i}, Z_{j}\right)=\rho, \quad i \neq j$, cuja FGP é dada por $G_{Y}(t)=1-\alpha+\alpha t$. Então, neste caso particular, de (2.13) obtemos a FGP de $S_{n}=Z_{1}+\ldots+Z_{n}$, que é dada por

$$
G_{S_{n}}(t)=\rho\left(1-\alpha+\alpha t^{n}\right)+(1-\rho)(1-\alpha+\alpha t)^{n} .
$$

A partir de (2.10) e (2.12) as expressões para a média e variância são dadas por

$$
\begin{aligned}
\mathbb{E}\left(S_{n}\right) & =n \alpha \\
\operatorname{Var}\left(S_{n}\right) & =n \alpha(1-\alpha)[1+(n-1) \rho] .
\end{aligned}
$$

Agora, calculemos a FGP de $S_{N}=Z_{1}+\cdots+Z_{N}$. Com as considerações usadas em (2.13) e utilizando (2.16), temos

$$
\begin{aligned}
G_{S_{N}}(t) & =\mathbb{E}\left(t^{S_{N}}\right)=\mathbb{E}\left[\mathbb{E}\left(t^{Z_{1}+\ldots+Z_{N}} \mid N\right)\right] \\
& =\sum_{n=0}^{\infty} \mathbb{E}\left(t^{S_{n}}\right) \mathbb{P}(N=n) \\
& =\sum_{n=0}^{\infty}\left[\rho\left(1-\alpha+\alpha t^{n}\right)+(1-\rho)(1-\alpha+\alpha t)^{n}\right] p_{n}
\end{aligned}
$$

Portanto, a FGP de $S_{N}$ é dada por

$$
G_{S_{N}}(t)=\rho\left[1-\alpha+\alpha G_{N}(t)\right]+(1-\rho) G_{N}(1-\alpha+\alpha t) .
$$

A última relação mostra que $S_{N}$ pode ser representada como uma média ponderada de um ponto de massa concentrada em zero e das distribuições de $N$ e $\sum_{i=1}^{N} W_{i}$, sendo que as $W_{i}$ 's são v.a.'s independentes e identicamente distribuídas Bernoulli com parâmetro $\alpha$.

Como conseqüência de (2.16), $S_{n}$ pode ser representada pela seguinte mistura de v.a.'s

$$
S_{n} \stackrel{d}{=} \rho X_{1}+(1-\rho) X_{2}
$$


A partir desta expressão e de (2.16) podemos facilmente observar que a distribuição de probabilidade de $S_{n}$ é uma distribuição composta de uma variável binomial com média $n \alpha$ e variância $n \alpha(1-\alpha)$, i.e., $X_{2} \sim \operatorname{Bi}(n, \alpha)$, com peso $(1-\rho)$ e a variável Bernoulli tem parâmetro $\alpha$, i.e., $X_{1} \sim B e(\alpha)$ re-escalada, tomando valores 0 e $n$, de média $n \alpha$, com peso $\rho$.

Por outro lado, da definição da FGP, temos que

$$
G_{S_{n}}(t)=\sum_{i=1}^{n} \mathbb{P}\left(S_{n}=i\right) t^{i} .
$$

Igualando termo a termo as equações (2.16) e (2.18), podemos escrever a distribuição exata da soma agregada $S_{n}$ como

$$
\begin{aligned}
& \mathbb{P}\left(S_{n}=0\right)=(1-\rho)(1-\alpha)^{n}+\rho(1-\alpha) ; \\
& \mathbb{P}\left(S_{n}=j\right)=(1-\rho)\left(\begin{array}{c}
n \\
j
\end{array}\right) \alpha^{j}(1-\alpha)^{n-j}, \quad \text { para } \quad j=1,2, \ldots, n-1 ; \\
& \mathbb{P}\left(S_{n}=n\right)=(1-\rho) \alpha^{n}+\rho \alpha .
\end{aligned}
$$

Observemos que $S_{n}$ representa a soma de $n$ variáveis Bernoulli equicorrelacionadas com a mesma distribuição.

Assumindo em (2.19) $\rho<0$, temos que

$$
\begin{aligned}
\mathbb{P}\left(S_{n}=0\right) \geq 0 & \Leftrightarrow(1-\rho)(1-\alpha)^{n}+\rho(1-\alpha) \geq 0 \\
& \Leftrightarrow(1-\rho)(1-\alpha)^{n-1}+\rho \geq 0 \\
& \Leftrightarrow \alpha \leq 1-\left(-\frac{\rho}{1-\rho}\right)^{\left(\frac{1}{n-1}\right)} .
\end{aligned}
$$

Analogamente obtemos

$$
\begin{aligned}
\mathbb{P}\left(S_{n}=n\right) \geq 0 & \Leftrightarrow(1-\rho) \alpha^{n}+\rho \alpha \geq 0 \\
& \Leftrightarrow(1-\rho) \alpha^{n-1}+\rho \geq 0 \\
& \Leftrightarrow \alpha \geq\left(-\frac{\rho}{1-\rho}\right)^{\left(\frac{1}{n-1}\right)} .
\end{aligned}
$$


Portanto, temos que

$$
\left(-\frac{\rho}{1-\rho}\right)^{\left(\frac{1}{n-1}\right)} \leq \alpha \leq 1-\left(-\frac{\rho}{1-\rho}\right)^{\left(\frac{1}{n-1}\right)} .
$$

Podemos observar que a última relação é válida só quando $\rho \in\left[-\frac{1}{2^{(n-1)}-1}, 0\right)$.

A distribuição de $S_{n}$ foi dada primeiro por Tallis (1962). Depois foi redescoberta por Luceño (1995) e denominada como distribuição binomial correlacionada com parâmetros $n, \alpha$ e $\rho$, sendo denotada por $S_{n} \sim \operatorname{CBi}(n, \alpha, \rho)$. Essa distribuição contém a distribuição binomial $B i(n, \alpha)$ no caso particular de $\rho=0$.

\subsection{Uma aplicação em resseguro do tipo stop- loss}

Consideremos um contrato de resseguro do tipo "stop-loss" com retenção $M>0$ (veja Figura 2.2).

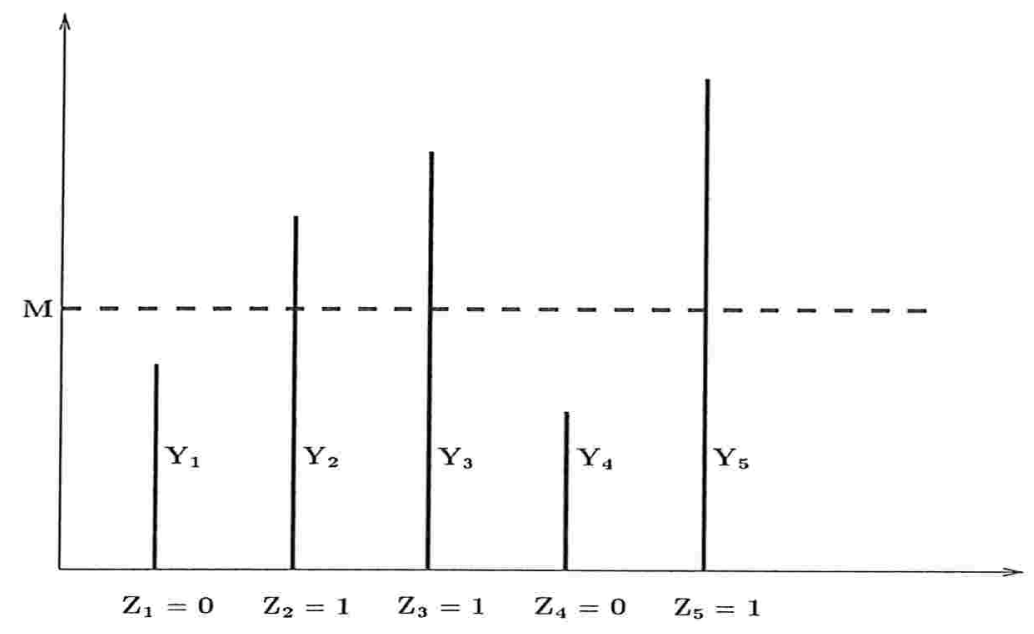

Figura. 2.2: Contrato de resseguro do tipo "stop-loss". 
Para todo sinistro $Y_{i}$ e $M$ fixo, as companhias de seguro e resseguro pagam $\min \left(Y_{i}, M\right)$ e $\max \left(Y_{i}-M, 0\right)$, respectivamente; veja. Daykin et al. (1994). Por exemplo, para os sinistros dados na Figura 2.2, a companhia de seguro deve pagar $Y_{1}, M, M, Y_{4}$ e $M$, sendo que a companhia de resseguro paga $0, Y_{2}-M, Y_{3}-M$, 0 e $Y_{5}-M$.

Denotemos por $N_{R}$ o número de sinistros que a resseguradora deve pagar, i.e.,

$$
N_{R}=\sum_{i=1}^{N} 1\left\{Y_{i}>M\right\}
$$

e seja $\gamma=\mathbb{P}\left(Y_{i}>M\right)$. As v.a.'s $Z_{i}=1\left\{Y_{i}>M\right\}$ têm a mesma distribuição Bernoulli com parâmetro $\gamma \in[0,1]$ e FGP dada por $G_{Z}(t)=1-\gamma+\gamma t$.

Lembremos que $Y_{1}, Y_{2}, \ldots, Y_{n}$ são igualmente correlacionadas e identicamente distribuídas com $\operatorname{Corr}\left(Y_{i}, Y_{j}\right)=\rho, i \neq j$. Então, a seqüência de v.a.'s Bernoulli $Z_{1}, Z_{2}, \ldots, Z_{n}$ é permutável e portanto $\operatorname{Corr}\left(Z_{i}, Z_{j}\right)=\rho_{1}, \operatorname{com} i \neq j$, para algum $\rho_{1} \in\left[-\frac{1}{n-1}, 1\right]$, de acordo com (2.4). O seguinte resultado dá a condição necessária e suficiente para afirmar que $\rho=\rho_{1}$.

\section{Lema 2.1.}

$\operatorname{Seja} \operatorname{Corr}\left(Y_{i}, Y_{j}\right)=\rho$, para $i \neq j e$

$$
\rho \in\left[\max \left\{-\frac{\gamma}{1-\gamma},-\frac{1-\gamma}{\gamma},-\frac{1}{n-1}\right\}, 1\right] .
$$

Então, $\operatorname{Corr}\left(Z_{i}, Z_{j}\right)=\rho, i \neq j$ se e somente se

$$
\mathbb{P}\left(Z_{i}=1 \mid Z_{j}=1\right)=\gamma+(1-\gamma) \rho \quad \text { e } \mathbb{P}\left(Z_{i}=0 \mid Z_{j}=0\right)=(1-\gamma)+\gamma \rho
$$

\section{Prova:}

As v.a.'s $Z_{i}, i=1,2, \ldots, n$, são identicamente distribuídas Bernoulli com parâmetro $\gamma$, esperança dada por $\mathbb{E}(Z)=\gamma=\mathbb{P}\left(Y_{i}>M\right)$ e variância dada por $\operatorname{Var}(Z)=$ $\gamma(1-\gamma)$. Então, $\operatorname{Corr}\left(Z_{i}, Z_{j}\right)=\rho$, para $i \neq j$, se e somente se, $\operatorname{Cov}\left(Z_{i}, Z_{j}\right)=$ 
$\rho \operatorname{Var}(Z)$, ou seja, $\mathbb{E}\left(Z_{i} Z_{j}\right)-\gamma^{2}=\gamma(1-\gamma) \rho$. Por outro lado, temos

$$
\mathbb{E}\left(Z_{i} Z_{j}\right)=1.1 . \mathbb{P}\left\{Z_{i}=1, Z_{j}=1\right\}=\mathbb{P}\left\{Z_{i}=1, Z_{j}=1\right\} .
$$

Portanto, $\mathbb{P}\left\{Z_{i}=1, Z_{j}=1\right\}=\gamma^{2}+\gamma(1-\gamma) \rho$ e pela fórmula de probabilidade condicional obtemos $\mathbb{P}\left(Z_{i}=1 \mid Z_{j}=1\right)=\gamma+(1-\gamma) \rho$.

A segunda relação em (2.20) segue do fato de que as variáveis $Z_{i}^{*}=1-Z_{i}$, $i=1,2, \ldots$ são também v.a's Bernoulli, com $\mathbb{P}\left\{Z_{i}^{*}=0\right\}=\gamma, \mathbb{P}\left\{Z_{i}^{*}=1\right\}=1-\gamma$, e

$$
\operatorname{Corr}\left(Z_{i}^{*}, Z_{j}^{*}\right)=\frac{\mathbb{E}\left[\left(1-Z_{i}^{*}\right)\left(1-Z_{j}^{*}\right)\right]-(1-\gamma)^{2}}{\gamma(1-\gamma)}=\rho
$$

As probabilidades condicionais (2.20) são não negativas, i.e., $\rho \geq \max \left(-\frac{\gamma}{1-\gamma},-\frac{1-\gamma}{\gamma}\right)$ e, da expressão dada em (2.4), temos que $\rho \geq-\frac{1}{n-1}$.

Portanto, $\rho \in\left[\max \left\{-\frac{\gamma}{1-\gamma},-\frac{1-\gamma}{\gamma},-\frac{1}{n-1}\right\}, 1\right]$. Então, as expressões em (2.20) são válidas.

Observemos que as v.a.'s $Z_{i}$ são equicorrelacionadas e permutáveis. As probabilidades condicionais (2.20) mostram que quando há existência de uma estrutura de correlação entre as observações (sinistros) originais $Y_{i}$, ela se conserva para as variáveis indicadoras dado o coeficiente de correlação $\rho$ e $\gamma=\mathbb{P}\left(Y_{i}>M\right)$.

Usando (2.16), obtemos a FGP de $N_{R}$ (número de sinistros que a companhia de resseguro deve pagar)

$$
G_{N_{R}}(t)=(1-\gamma) \rho+\gamma \rho G_{N}(t)+(1-\rho) G_{N}(1-\gamma+\gamma t)
$$

\section{Exemplos:}

1. Se $N$ tem distribuição Poisson com parâmetro $\lambda>0$, de (2.21) temos

$$
G_{N_{R}}(t)=(1-\gamma) \rho+\gamma \rho e^{\lambda(t-1)}+(1-\rho) e^{\lambda(\gamma t-1)} .
$$


2. Se $N$ possui distribuição Binomial com parâmetros $n$ e $p$, de (2.21) temos

$$
G_{N_{R}}(t)=(1-\gamma) \rho+\gamma \rho(1-p+p t)^{n}+(1-\rho)(1-p \gamma+p \gamma t)
$$

Lembremos que num contrato de seguro do tipo "stop-loss" com retenção $M$, a resseguradora deve pagar $Y_{R}=\max \{Y-M, 0\}$, com $M$ sendo um inteiro positivo, $M<k$. De (2.2), obtemos a seguinte distribuição para $Y_{R}$ :

$$
\mathbb{P}\left(Y_{R}=0\right)=1-\gamma \quad \text { e } \quad \mathbb{P}\left(Y_{R}=i-M\right)=\pi_{i}, \quad i=M+1, \ldots, k
$$

Portanto, a FGP de $Y_{R}$ é dada por

$$
G_{Y_{R}}(t)=\mathbb{E}\left(t^{Y_{R}}\right)=1-\gamma+\sum_{r=M+1}^{k} \pi_{r} t^{r-M}
$$

Depois que substituimos $G_{N}(t)$ por $G_{N_{R}}(t)$ e $G_{Y}(t)$ por $G_{Y_{R}}(t)$ em (2.13), conseguimos a distribuição das observações acumuladas

$$
S_{N_{R}}=\sum_{i=1}^{N_{R}} \max \left\{Y_{i}-M, 0\right\}
$$

Para a resseguradora, a FGP do número de observações que a resseguradora deve pagar é dada por

$$
G_{S_{N_{R}}}(t)=\rho(1-\gamma)+\rho \sum_{r=M+1}^{k} \pi_{r} G_{N_{R}}\left(t^{r}\right)+(1-\rho) G_{N_{R}}\left(G_{Y_{R}}(t)\right)
$$

Se $N=n=$ constante, então $G_{N}(t)=t^{n}$ e (2.21) tem a forma

$$
G_{n_{R}}(t)=\rho\left(1-\gamma+\gamma t^{n}\right)+(1-\rho)(1-\gamma+\gamma t)^{n}
$$

A v.a. $n_{R}$ é o número de elementos dentre $n$ observações igualmente correlacionadas que possuem valor maior que a retenção especificada $M$. Portanto, dizemos que $S_{n_{R}} \sim \operatorname{CBi}(n, \gamma, \rho)$. 
Neste capítulo, estudamos seqüências de v.a.'s igualmente correlacionadas. Foram apresentados alguns resultados de permutabilidade que se restringe à possível estrutura de correlação para seqüências de v.a.'s com os dois primeiros momentos finitos. Além disso, foram introduzidos os modelos do vetor aleatório dependente que foi focalizado neste trabalho em (2.6) e (2.7). Como conseqüência, obtivemos a FGP da soma aleatória (2.1), que estende o resultado clássico. Em seguida, foram estendidas algumas de suas propriedades estatísticas e apresentada uma aplicação em seguros do tipo "stop-loss". 


\section{Capítulo 3}

\section{Correlação entre riscos}

\section{dependentes}

Para medir a dependência de duas v.a.'s é comum usar o coeficiente de correlação linear, que é uma medida de dependência linear. Embora ele não seja sempre uma medida apropriada para capturar completamente a relação de dependência entre duas variáveis, ao longo deste capítulo, nossa análise estará baseada nesta medida, além dos resultados principais do capítulo 2 .

Este capítulo está dividido como segue. Na seção 3.1, daremos uma breve motivação e mostraremos um exemplo simples no caso de v.a.'s Poisson. Na seção 3.2, investigaremos mudanças no prêmio que podem surgir a partir da interdependência de diferentes linhas de seguro. Na seção 3.3, mostraremos que os modelos correspondentes aos riscos dependentes são modelos de super-dispersão relativo ao modelo de independência. Finalmente, na seção 3.4, introduziremos o prêmio do tipo "stop-loss" e o ilustraremos com um exemplo. 


\subsection{Motivação}

Como vimos anteriormente, o interesse básico das companhias de seguros é analisar os riscos acumulados. Já vimos que, na prática, os riscos exibem dependência mútua e a suposição de independência pode ser usada como uma primeira aproximação do valor verdadeiro do prêmio. Para quantificar o grau de dependência de dois riscos acumulados, usamos o coeficiente de correlação entre as freqüências e entre os próprios valores dos sinistros.

Sob as mesmas condições dadas no capítulo 2, redefinamos a soma aleatória (2.1) da seguinte maneira. Consideremos $X$ uma v.a. que representa o valor do sinistro de seguro e $N_{X}$ a quantidade de ocorrências anuais que sofreram o sinistro, i.e., a freqüência dos sinistros. Então, a soma acumulada (agregada) é definida como em (2.1) por

$$
S_{X} \stackrel{d}{=} \sum_{i=1}^{N_{X}} X_{i}
$$

com $N_{X}$ independente dos $X_{i}$ 's e a seqüência $\left\{X_{i}\right\}$ sendo v.a.'s identicamente distribuídas, equicorrelacionadas, não negativas e que assumem valores inteiros, com distribuição dada por

$$
P(X=r)=\pi_{r}, \quad r=0,1, \ldots, k, \quad \sum_{r=0}^{k} \pi_{r}=1,
$$

de média e variância finitas, tal que $\operatorname{Corr}\left(X_{i}, X_{l}\right)=\rho_{X}>0$, para $i \neq l$.

A interpretação é simples: $X_{i}$ denota o valor do $i$-ésimo sinistro, $N_{X}$ representa o número de sinistros num período de tempo fixado e $S_{X}$ indica a quantidade de sinistros acumulados neste período; ver e.g. Bowers et al. (1997).

Consideremos uma outra seqüência $\left\{Y_{j}\right\}$ de v.a.'s não negativas e identicamente distribuídas, equicorrelacionadas, $\operatorname{com} \operatorname{Corr}\left(Y_{j}, Y_{l}\right)=\rho_{Y}>0$, para $j \neq l$. A 
seqüência $\left\{X_{i}\right\}$ é independente da seqüência $\left\{Y_{j}\right\}$. Denotemos por $S_{Y}=\sum_{j=1}^{N_{Y}} Y_{j}$ a soma aleatória correspondente, com $N_{Y}$ independente dos $Y_{j}$ 's.

Neste capítulo, estamos interessados em obter a expressão da correlação entre somas agregadas $S_{X}$ e $S_{Y}$ como uma função das correlações entre as freqüências dos sinistros $N_{X}$ e $N_{Y}$ e das correlações entre os próprios valores dos sinistros $X_{i}$ 's e $Y_{j}$ 's.

\section{Exemplo:}

Sejam $N_{X}$ e $N_{Y}$ v.a.'s Poisson e assumamos que $(X, Y),\left(X, N_{X}\right)$ e $\left(Y, N_{Y}\right)$ são mutuamente independentes. Sob estas condições, queremos obter a correlação entre $S_{X}$ e $S_{Y}$.

Por definição, temos que

$$
\rho_{S_{X}, S_{Y}}=\frac{\operatorname{Cov}\left(S_{X}, S_{Y}\right)}{\sqrt{\operatorname{Var}\left(S_{X}\right)} \sqrt{\operatorname{Var}\left(S_{Y}\right)}} .
$$

Considerando as condições de independência, $\rho=0$, e usando (2.15), temos que

$$
\begin{aligned}
& \operatorname{Var}\left(S_{X}\right)=\mathbb{E}\left(N_{X}\right) \operatorname{Var}(X)+\operatorname{Var}\left(N_{X}\right) \mathbb{E}^{2}(X), \\
& \operatorname{Var}\left(S_{Y}\right)=\mathbb{E}\left(N_{Y}\right) \operatorname{Var}(Y)+\operatorname{Var}\left(N_{Y}\right) \mathbb{E}^{2}(Y) .
\end{aligned}
$$

Como $N_{X}$ e $N_{Y}$ são v.a.'s Poisson, então

$$
\begin{aligned}
& \operatorname{Var}\left(S_{X}\right)=\mathbb{E}\left(N_{X}\right) \mathbb{E}\left(X^{2}\right), \\
& \operatorname{Var}\left(S_{Y}\right)=\mathbb{E}\left(N_{Y}\right) \mathbb{E}\left(Y^{2}\right) .
\end{aligned}
$$

Por outo lado,

$$
\begin{aligned}
\operatorname{Cov}\left(S_{X}, S_{Y}\right) & =\mathbb{E}\left(S_{X} S_{Y}\right)-\mathbb{E}\left(S_{Y}\right) \mathbb{E}\left(S_{Y}\right) \\
& =\mathbb{E}(X) \mathbb{E}(Y)\left[\mathbb{E}\left(N_{X} N_{Y}\right)-\mathbb{E}\left(N_{X}\right) \mathbb{E}\left(N_{Y}\right)\right] \\
& =\mathbb{E}(X) \mathbb{E}(Y) \operatorname{Cov}\left(N_{X}, N_{Y}\right) .
\end{aligned}
$$


Substituindo em (3.1), obtemos

$$
\rho_{S_{X}, S_{Y}}=\frac{\mathbb{E}(X) \mathbb{E}(Y)}{\sqrt{\mathbb{E}\left(X^{2}\right)} \sqrt{\mathbb{E}\left(Y^{2}\right)}} \rho_{N_{X}, N_{Y}},
$$

que é a relação que procuramos.

Ao longo deste capítulo, calcularemos também as esperanças e variâncias das somas agregadas correspondentes, que são a base para o cálculo do prêmio de risco puro.

\subsection{Correlação entre somas dependentes}

Sem perda de generalidade, assumamos duas linhas de seguros e consideremos as somas aleatórias $S_{X}$ e $S_{Y}$ correspondentes as v.a.'s $X, N_{X}$ e $Y, N_{Y}$, respectivamente.

Seja $\operatorname{Corr}\left(N_{X}, N_{Y}\right)=\rho_{N_{X}, N_{Y}}>0$ a correlação das freqüências dos sinistros, que mede o grau de dependência entre o número de ocorrências $\left(N_{X}\right.$ e $\left.N_{Y}\right)$ dos sinistros $X_{i}$ e $Y_{j}$ de duas linhas de seguro. Nosso objetivo é calcular a correlação $\rho_{S_{X}, S_{Y}}$ entre as somas acumuladas $S_{X}$ e $S_{Y}$, que será uma função das correlações entre os números de ocorrências de duas linhas de seguros $\rho_{N_{X}, N_{Y}}$ e das correlações dos sinistros de cada companhia $\rho_{X}=\operatorname{Corr}\left(X_{i}, X_{l}\right)$ e $\rho_{Y}=\operatorname{Corr}\left(Y_{j}, Y_{l}\right)$. O seguinte resultado é verdadeiro.

\section{Teorema 3.1.}

Sob as suposições acima, a correlação $\rho_{S_{X}, S_{Y}}$ entre as somas agregadas $S_{X}$ e $S_{Y}$ é dada por:

$$
\rho_{S_{X}, S_{Y}}=\frac{\mathbb{E}(X) \mathbb{E}(Y)}{\sqrt{\frac{\operatorname{Var}\left(S_{X}\right)}{\operatorname{Var}\left(N_{X}\right)} \frac{\operatorname{Var}\left(S_{Y}\right)}{\operatorname{Var}\left(N_{Y}\right)}}} \rho_{N_{X}, N_{Y}}
$$


sendo que as variâncias $\operatorname{Var}\left(S_{X}\right)$ e $\operatorname{Var}\left(S_{Y}\right)$ são dadas em (2.15).

\section{Prova:}

Pela definição de correlação, temos que

$$
\rho_{S_{X}, S_{Y}}=\frac{\operatorname{Cov}\left(S_{X}, S_{Y}\right)}{\sqrt{\operatorname{Var}\left(S_{X}\right) \operatorname{Var}\left(S_{Y}\right)}}
$$

com

$$
\operatorname{Cov}\left(S_{X}, S_{Y}\right)=\mathbb{E}\left(S_{X} S_{Y}\right)-\mathbb{E}\left(S_{X}\right) \mathbb{E}\left(S_{Y}\right)
$$

Seja $Z_{k}=X_{i} Y_{j}, \operatorname{com} i=1,2, \ldots, N_{X}, j=1,2, \ldots, N_{Y}$ e $k=1,2, \ldots, N_{X} N_{Y}$. Então

$$
\begin{aligned}
\mathbb{E}\left(S_{X} S_{Y}\right) & =\mathbb{E}\left(\sum_{k=1}^{N_{X} N_{Y}} Z_{k}\right)=\mathbb{E}\left(Z_{k}\right) \mathbb{E}\left(N_{X} N_{Y}\right) \\
& =\mathbb{E}(X) \mathbb{E}(Y) \mathbb{E}\left(N_{X} N_{Y}\right) .
\end{aligned}
$$

Usando (2.12) e esta última expressão em (3.3), temos que

$$
\operatorname{Cov}\left(S_{X}, S_{Y}\right)=\mathbb{E}(X) \mathbb{E}(Y) \operatorname{Cov}\left(N_{X}, N_{Y}\right)
$$

Fazendo

$$
\frac{\operatorname{Cov}\left(S_{X}, S_{Y}\right)}{\sqrt{\operatorname{Var}\left(S_{X}\right) \operatorname{Var}\left(S_{X}\right)}}=\frac{\mathbb{E}(X) \mathbb{E}(Y)}{\frac{\sqrt{\operatorname{Var}\left(S_{X}\right) \operatorname{Var}\left(S_{X}\right)}}{\sqrt{\operatorname{Var}\left(N_{X}\right) \operatorname{Var}\left(N_{X}\right)}}} \frac{\operatorname{Cov}\left(N_{X}, N_{Y}\right)}{\sqrt{\operatorname{Var}\left(N_{X}\right) \operatorname{Var}\left(N_{X}\right)}},
$$

temos que a correlação $\rho_{S_{X}, S_{Y}}$ é dada por

$$
\rho_{S_{X}, S_{Y}}=\frac{\mathbb{E}(X) \mathbb{E}(Y)}{\sqrt{\frac{\operatorname{Var}\left(S_{X}\right)}{\operatorname{Var}\left(N_{X}\right)} \frac{\operatorname{Var}\left(S_{Y}\right)}{\operatorname{Var}\left(N_{Y}\right)}}} \rho_{N_{X}, N_{Y}}
$$

Assim, fica demonstrado o Teorema 3.1.

Observemos que $\rho_{S_{X}, S_{Y}}$ depende dos valores de $\operatorname{Var}\left(N_{X}\right), \operatorname{Var}\left(N_{Y}\right), \operatorname{Var}\left(S_{X}\right)$ e $\operatorname{Var}\left(S_{Y}\right)$, i.e., de $\rho_{X}$, de $\rho_{Y}$ e de $\rho_{N_{X}, N_{Y}}$. Nossas considerações são uma extensão do 
modelo proposto por Mirzai (1999) que tem aplicações na troca de risco dentro de companhias de seguro e resseguro. O resultado principal (Teorema 3.1) e aplicações estão publicados em Paiva e Kolev (2001a).

\subsection{Modelos de super-dispersão}

A seguir, compararemos três situações: primeiro mostraremos o caso de independência (Caso 0) e depois apresentaremos duas versões que consideram diferentes tipos de dependência (Casos 1 e 2). Denotemos por $S_{i n d}, S_{d e p 1}$ e $S_{d e p 2}$ a soma de $S_{X}$ e $S_{Y}$ em cada um dos casos, respectivamente.

Caso 0 (independência): Neste caso, consideraremos que existe independência entre os sinistros e o número de ocorrências de sinistros de duas linhas de seguro. Assim, temos $\rho_{X}=0, \rho_{Y}=0$ e $\rho_{N_{X}, N_{Y}}=0$. As expressões para $\mathbb{E}\left(S_{\text {ind }}\right)$ e $\operatorname{Var}\left(S_{\text {ind }}\right)$ são

$$
\begin{aligned}
\mathbb{E}\left(S_{\text {ind }}\right)= & \mathbb{E}\left(S_{X}+S_{Y}\right)=\mathbb{E}\left(N_{X}\right) \mathbb{E}(X)+\mathbb{E}\left(N_{Y}\right) \mathbb{E}(Y) \\
\operatorname{Var}\left(S_{\text {ind }}\right)= & \operatorname{Var}\left(S_{X}+S_{Y}\right)=\operatorname{Var}\left(S_{X}\right)+\operatorname{Var}\left(S_{Y}\right) \\
= & \mathbb{E}\left(N_{X}\right) \operatorname{Var}(X)+\operatorname{Var}\left(N_{X}\right) \mathbb{E}^{2}(X)+\mathbb{E}\left(N_{Y}\right) \operatorname{Var}(Y) \\
& +\operatorname{Var}\left(N_{Y}\right) \mathbb{E}^{2}(Y) .
\end{aligned}
$$

Caso 1 (dependência entre freqüências): Assumamos que existe dependência somente entre o número de ocorrências de sinistros, $N_{X}$ e $N_{Y}$. Ou seja,

$$
\rho_{S_{X} S_{Y}}=f\left(\rho_{N_{X} N_{Y}}\right)>0
$$

para alguma função contínua $f(\cdot)$, i.e., 
- $(X, Y),\left(X, N_{X}\right),\left(Y, N_{Y}\right), X_{i}$ 's e $Y_{j}$ 's são independentes;

- $\operatorname{Corr}\left(N_{X}, N_{Y}\right)=\rho_{N_{X} N_{Y}}>0$.

Usando (2.14) e (2.15), temos que

$$
\begin{aligned}
\mathbb{E}\left(S_{\text {dep } 1}\right)= & \mathbb{E}\left(S_{X}+S_{Y}\right)=\mathbb{E}\left(N_{X}\right) \mathbb{E}(X)+\mathbb{E}\left(N_{Y}\right) \mathbb{E}(Y) \\
\operatorname{Var}\left(S_{\text {dep } 1}\right)= & \operatorname{Var}\left(S_{X}\right)+\operatorname{Var}\left(S_{Y}\right)+2 \operatorname{Cov}\left(S_{X}, S_{Y}\right) \\
= & \mathbb{E}\left(N_{X}\right) \operatorname{Var}(X)+\operatorname{Var}\left(N_{X}\right) \mathbb{E}^{2}(X)+\mathbb{E}\left(N_{Y}\right) \operatorname{Var}(Y) \\
& +\operatorname{Var}\left(N_{Y}\right) \mathbb{E}^{2}(Y)+2 \mathbb{E}(X) \mathbb{E}(Y) \operatorname{Cov}\left(N_{X}, N_{Y}\right)
\end{aligned}
$$

Observação 1: Comparando (3.5)-(3.8), observamos que $\mathbb{E}\left(S_{\text {ind }}\right)=\mathbb{E}\left(S_{\text {dep } 1}\right)$ e $\operatorname{Var}\left(S_{\text {ind }}\right)<\operatorname{Var}\left(S_{d e p 1}\right)$, se $\rho_{N_{X}, N_{Y}}>0$, i.e., o Caso 1 é um modelo de superdispersão relativo ao modelo de riscos independentes.

Caso 2 (dependência geral): Consideremos que existe dependência entre o número de ocorrências de sinistros $N_{X}$ e $N_{Y}$ e entre os próprios sinistros $X_{i}$ 's e $Y_{j}^{\prime}$ 's de cada companhia de seguro, i.e.,

$$
\rho_{S_{X}, S_{Y}}=g\left(\rho_{N_{X} N_{Y}}, \rho_{X}, \rho_{Y}\right)>0
$$

para alguma função contínua $g(\cdot)$. Então, neste caso temos:

- $(X, Y),\left(X, N_{X}\right),\left(Y, N_{Y}\right)$ são independentes;

- $\operatorname{Corr}\left(X_{i}, X_{l}\right)=\rho_{X}>0$, com $i \neq l$;

- $\operatorname{Corr}\left(Y_{j}, Y_{l}\right)=\rho_{Y}>0, \quad$ com $j \neq l$;

- $\operatorname{Corr}\left(N_{X}, N_{Y}\right)=\rho_{N_{X}, N_{Y}}>0$. 
De (2.14) e (2.15), obtemos as expressões para a esperança e variância

$$
\begin{aligned}
\mathbb{E}\left(S_{\text {dep } 2}\right)= & \mathbb{E}\left(S_{X}+S_{Y}\right)=\mathbb{E}\left(N_{X}\right) \mathbb{E}(X)+\mathbb{E}\left(N_{Y}\right) \mathbb{E}(Y) \\
\operatorname{Var}\left(S_{\text {dep } 2}\right)= & \operatorname{Var}\left(S_{X}\right)+\operatorname{Var}\left(S_{Y}\right)+2 \operatorname{Cov}\left(S_{X}, S_{Y}\right) \\
= & \mathbb{E}\left(N_{X}\right) \operatorname{Var}(X)+\operatorname{Var}\left(N_{X}\right) \mathbb{E}^{2}(X)+\rho_{X} \operatorname{Var}(X) \mathbb{E}\left[N_{X}\left(N_{X}-1\right)\right] \\
& +\mathbb{E}\left(N_{Y}\right) \operatorname{Var}(Y)+\operatorname{Var}\left(N_{Y}\right) \mathbb{E}^{2}(Y)+\rho_{Y} \operatorname{Var}(Y) \mathbb{E}\left[N_{Y}\left(N_{Y}-1\right)\right] \\
& +2 \mathbb{E}(X) \mathbb{E}(Y) \operatorname{Cov}\left(N_{X}, N_{Y}\right) .
\end{aligned}
$$

Observação 2: Comparando (3.7)-(3.10), vemos que $\mathbb{E}\left(S_{\text {dep } 1}\right)=\mathbb{E}\left(S_{\text {dep } 2}\right)$, mas $\operatorname{Var}\left(S_{d e p 1}\right)<\operatorname{Var}\left(S_{d e p 2}\right)$, se $\rho_{X}>0$ e $\rho_{Y}>0$, i.e., o Caso 2 é um modelo de super-dispersão relativo ao Caso 1.

Em conseqüência das observações 1 e 2, temos que as esperanças dos casos considerados são as mesmas, i.e., $\mathbb{E}\left(S_{\text {ind }}\right)=\mathbb{E}\left(S_{\text {dep } 1}\right)=\mathbb{E}\left(S_{\text {dep } 2}\right)$, mas existem incrementos nas variâncias dados por $\Delta_{1}$ (Caso 1) e $\Delta_{2}$ (Caso 2), expressas a seguir

$$
\begin{aligned}
\Delta_{1} & =\operatorname{Var}\left[S_{\text {dep } 1}\right]-\operatorname{Var}\left[S_{\text {ind }}\right]=2 \mathbb{E}[X] \mathbb{E}[Y] \operatorname{Cov}\left(N_{X}, N_{Y}\right) \\
\Delta_{2} & =\operatorname{Var}\left[S_{d e p 2}\right]-\operatorname{Var}\left[S_{\text {dep } 1}\right] \\
& =\rho_{X} \operatorname{Var}(\mathrm{X}) \mathbb{E}\left[N_{X}\left(N_{X}-1\right)\right]+\rho_{Y} \operatorname{Var}(Y) \mathbb{E}\left[N_{Y}\left(N_{Y}-1\right)\right] .
\end{aligned}
$$

Este fato implica que as distribuições de $S_{d e p 1}$ e $S_{d e p 2}$ possuem caudas mais pesadas que as caudas da distribuição de $S_{X}+S_{Y}$ no caso de independência. As mudanças mencionadas fornecem um incremento no prêmio que usualmente é uma função de esperanças e variâncias. 


\subsection{Classificação de riscos dependentes}

Quando o número de sinistros $N_{X}$ e dos $X_{i}$ 's são independentes, o prêmio de risco puro (para companhias de seguro) $\mathbb{E}\left(S_{X}\right)$ é dado por:

$$
\mathbb{E}\left(S_{X}\right)=\mathbb{E}\left(\sum_{n=1}^{N_{X}} X_{n}\right)=\mathbb{E}\left(N_{X}\right) \mathbb{E}(X) .
$$

Consideremos um contrato de resseguro individual com retenção $M>0$. $\mathrm{O}$ prêmio do tipo "stop-loss" é a perda esperada

$$
\mathbb{E}\left[\mathcal{L}_{M}(X)\right]=\mathbb{E}[\max (X-M, 0)] .
$$

Esta relação mostra que a companhia de resseguro paga 0 reais, se o sinistro $X \leq M$ e paga a quantidade $X-M$, se $X>M$. Na prática, dependendo do contrato, o prêmio do tipo "stop-loss" pode ser utilizado tanto para sinistros individuais $X_{i}$ 's como para a soma acumulada $S_{X}$, i.e.,

$$
\mathbb{E}\left[\mathcal{L}_{M}\left(S_{X}\right)\right]=\mathbb{E}\left[\mathcal{L}_{M}\left(\sum_{i=1}^{N_{X}} X_{i}\right)\right]=\mathbb{E}\left[\sum_{i=1}^{N_{X}} \max \left(X_{i}-M, 0\right)\right]
$$

Um outro tipo do prêmio "stop-loss" é $\mathbb{E}\left[\mathcal{L}_{M, L}\left(S_{X}\right)\right]$, que é a perda esperada com retenção $M$ e limite $L$ ( $M$ e $L$ fixos), em que

$$
\mathcal{L}_{M, L}(X)=\min \{\max (X-M, 0), L\} .
$$

Assim, temos:

$$
\mathcal{L}_{M, L}\left(S_{X}\right)= \begin{cases}0, & S_{X} \leq M \\ S_{X}-M, & M<S_{X} \leq M+L \\ L, & S_{X}>L+M\end{cases}
$$

Neste contrato, a companhia de resseguro deve pagar 0 , se $S_{X} \leq M$, a quantidade aleatória $S_{X}-M$, se $M<S_{X} \leq M+L$, e o limite $L$, se $S_{X}>L+M$; veja Figura 3.1. 


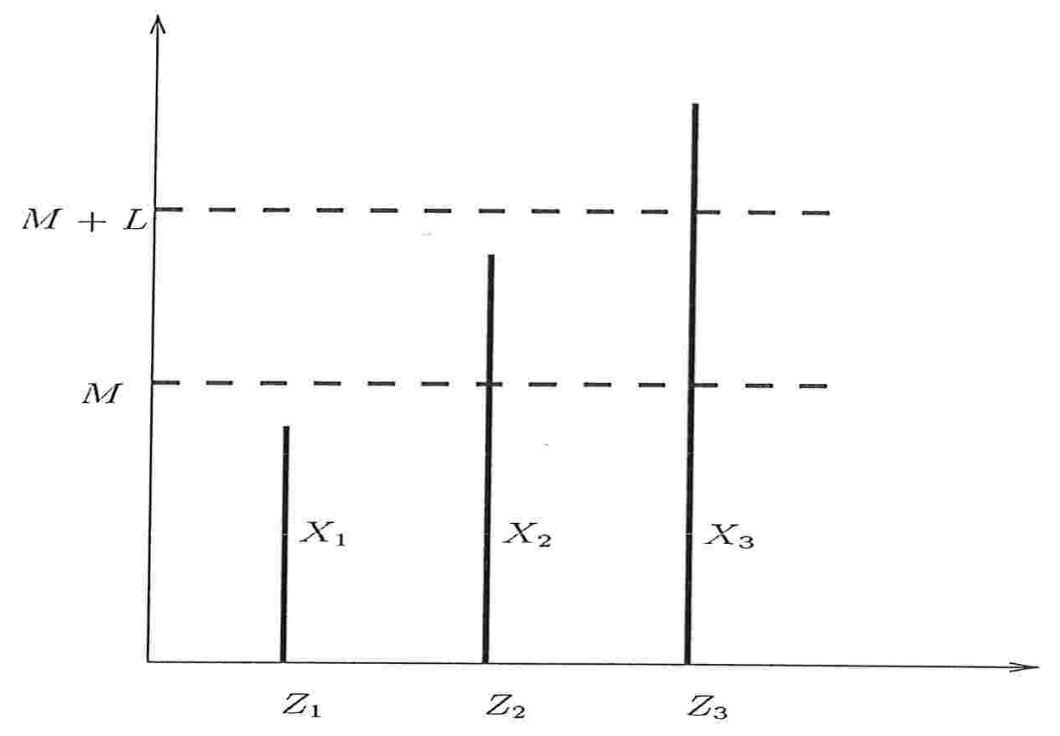

Figura 3.1: Contrato de resseguro do tipo "stop-loss".

Isto é, a companhia de resseguro paga $0, X_{2}-M$ e $L$ para os sinistros observados $X_{1}, X_{2}$ e $X_{3}$, respectivamente.

Logo, a perda esperada agregada numa faixa $[M, M+L]$ pode ser expressa como:

$$
\mathbb{E}\left[\mathcal{L}_{M, L}\left(S_{\text {ind }}\right)\right]=\mathbb{E}\left[\mathcal{L}_{M, L}\left(\sum_{i=1}^{N_{X}} X_{i}+\sum_{i=1}^{N_{Y}} Y_{i}\right)\right]
$$

\section{Exemplo}

Uma forma de considerar a dependência entre as freqüências de duas companhias de seguro é incorporar a seguinte estrutura. Denotemos por $\hat{N}_{x}$ e $\hat{N}_{y}$ as freqüências que são independentes da presença ou ausência de qualquer outro risco e $N_{X Y}$ a freqüência que é comum a ambos os riscos. Podemos representar $N_{X}$ e $N_{Y}$ como

$$
\left\{\begin{array}{l}
N_{X}=\hat{N}_{x}+N_{X Y} \\
N_{Y}=\hat{N}_{y}+N_{X Y}
\end{array}\right.
$$

Portanto, a perda esperada agregada correspondente a tal dependência para 
uma faixa com retenção $M$ e limite $L$ é dada por:

$$
\mathbb{E}\left[\mathcal{L}_{M, L}\left(S_{d e p}\right)\right]=\mathbb{E}\left[\mathcal{L}_{M, L}\left(\sum_{i=1}^{\hat{N}_{x}} X_{i}+\sum_{i=1}^{\hat{N}_{y}} Y_{i}+\sum_{i=\hat{N}_{x}+1}^{N_{x}} X_{i}+\sum_{i=\hat{N}_{y}+1}^{N_{y}} Y_{i}\right)\right]
$$

Suponhamos que:

- $\hat{N}_{x}, \hat{N}_{y}$ e $N_{X Y}$ são independentes;

- $\hat{N}_{x}, \hat{N}_{y}$ e $N_{X Y}$ têm distribuição Poisson, i.e., o vetor $\left(N_{X}, N_{Y}\right)$ têm distribuição Poisson bivariada (veja Johnson e Kotz (1997)).

Comparemos a perda esperada e a variância das somas aleatórias agregadas $S_{i n d}, S_{d e p 1}$ e $S_{d e p 2}$ no caso de que a companhia de seguro assume o risco total, i.e., $M=0$ e $L=\infty$. Este caso corresponde ao cálculo do risco puro para companhias de seguro. Assim, temos

$$
\begin{aligned}
\mathbb{E}\left[S_{\text {ind }}\right] & =\mathbb{E}\left[N_{X}\right] \mathbb{E}[X]+\mathbb{E}\left[N_{Y}\right] \mathbb{E}[Y] \\
\operatorname{Var}\left[S_{\text {ind }}\right] & =\mathbb{E}\left[N_{X}\right] \mathbb{E}\left[X^{2}\right]+\mathbb{E}\left[N_{Y}\right] \mathbb{E}\left[Y^{2}\right]
\end{aligned}
$$

Calculemos as esperanças e variâncias nos Casos 1 e 2 usando (2.14) e (2.15).

$$
\begin{aligned}
\mathbb{E}\left[S_{\text {dep } 1}\right]= & \mathbb{E}\left[\hat{N}_{x}\right] \mathbb{E}[X]+\mathbb{E}\left[\hat{N}_{y}\right] \mathbb{E}[Y]+\mathbb{E}\left[N_{X Y}\right] \mathbb{E}[X+Y] \\
\operatorname{Var}\left[S_{\text {dep } 1}\right]= & \left(\mathbb{E}\left[\hat{N}_{x}\right]+\mathbb{E}\left[N_{X Y}\right]\right) \mathbb{E}\left[X^{2}\right]+\left(\mathbb{E}\left[\hat{N}_{x}\right]+\mathbb{E}\left[N_{X Y}\right]\right) \mathbb{E}\left[Y^{2}\right] \\
& +2 \mathbb{E}\left[N_{X Y}\right] \mathbb{E}[X] \mathbb{E}[Y],
\end{aligned}
$$

e

$$
\mathbb{E}\left[S_{\text {dep } 2}\right]=\mathbb{E}\left[\hat{N}_{x}\right] \mathbb{E}[X]+\mathbb{E}\left[\hat{N}_{y}\right] \mathbb{E}[Y]+\mathbb{E}\left[N_{X Y}\right] \mathbb{E}[X+Y]
$$




$$
\begin{aligned}
\operatorname{Var}\left[S_{\text {dep } 2}\right]= & \left(\mathbb{E}\left[\hat{N}_{x}\right]+\mathbb{E}\left[N_{X Y}\right]\right) \mathbb{E}\left[X^{2}\right]+\left(\mathbb{E}\left[\hat{N}_{x}\right]\right. \\
& \left.+\mathbb{E}\left[N_{X Y}\right]\right) \mathbb{E}\left[Y^{2}\right]+2 \mathbb{E}\left[N_{X Y}\right] \mathbb{E}[X] \mathbb{E}[Y] \\
& +\rho_{X} \operatorname{Var}(X)\left[\mathbb{E}^{2}\left(\hat{N}_{x}\right)+\mathbb{E}^{2}\left(N_{X Y}\right)+2 \mathbb{E}\left(\hat{N}_{x}\right) \mathbb{E}\left(N_{X Y}\right)\right] \\
& +\rho_{Y} \operatorname{Var}(Y)\left[\mathbb{E}^{2}\left(\hat{N}_{y}\right)+\mathbb{E}^{2}\left(N_{X Y}\right)+2 \mathbb{E}\left(\hat{N}_{y}\right) \mathbb{E}\left(N_{X Y}\right)\right]
\end{aligned}
$$

Observemos também que a dependência entre as freqüências não muda o valor esperado de $S_{i n d}, S_{d e p 1}$ e $S_{d e p 2}$. Mas existem incrementos nas variâncias dados por:

$$
\begin{aligned}
\Delta_{1}= & \operatorname{Var}\left[S_{\text {dep } 1}\right]-\operatorname{Var}\left[S_{\text {ind }}\right]=2 \mathbb{E}\left[N_{X Y}\right] \mathbb{E}[X] \mathbb{E}[Y] \\
\Delta_{2}= & \operatorname{Var}\left[S_{\text {dep } 2}\right]-\operatorname{Var}\left[S_{\text {dep } 1}\right] \\
= & \rho_{X} \operatorname{Var}(X)\left[\mathbb{E}^{2}\left(\hat{N}_{x}\right)+\mathbb{E}^{2}\left(N_{X Y}\right)+2 \mathbb{E}\left(\hat{N}_{x}\right) \mathbb{E}\left(N_{X Y}\right)\right] \\
& +\rho_{Y} \operatorname{Var}(Y)\left[\mathbb{E}^{2}\left(\hat{N}_{y}\right)+\mathbb{E}^{2}\left(N_{X Y}\right)+2 \mathbb{E}\left(\hat{N}_{y}\right) \mathbb{E}\left(N_{X Y}\right)\right] .
\end{aligned}
$$

Isto confirma o fato de que as distribuições das somas aleatórias com dependência $\left(S_{d e p 1}\right.$ e $\left.S_{d e p 2}\right)$ possuem caudas mais pesadas do que as caudas da distribuição da soma sem considerar dependência $\left(S_{\text {ind }}\right)$.

Neste capítulo, podemos concluir que os modelos de riscos dependentes são modelos de super-dispersão relativos ao modelo de riscos independentes. 


\section{Capítulo 4}

\section{Processos INAR(1) e DAR(1) estendidos}

Em muitas situações surgem dados estatísticos que são expressos em termos de contagem tomados seqüencialmente no tempo e que são correlacionados. $\mathrm{Na}$ modelagem do processo de contagem com dependência, é natural considerar que a realização do processo $X_{t}$ é composta de sobreviventes dos elementos no instante anterior $X_{t-1}$ mais o incremento de um novo processo (inovação) $\epsilon_{t}$, que possui uma certa distribuição discreta.

Nos últimos anos, muitos autores têm trabalhado o problema de análise de séries de tempo de valores inteiros, e.g. MacDonald e Zucchini (1997), capítulo 1. Muitos modelos que têm sido sugeridos são baseados no operador de Stuetel e van Harn (1979), definido em (4.2).

Este capítulo está dividido como segue. Na seção 4.1, estenderemos o processo INAR(1) processo autoregressivo de ordem 1 que assume valores inteiros, estudado por Al-Osh e Alzaid (1987) e McKenzie (1988), considerando respostas binárias 
equicorrelacionadas envolvidas. Da mesma maneira, usando os resultados obtidos no capítulo 2, apresentaremos duas extensões do processo DAR(1), introduzido por Jacobs e Lewis (1978).

\subsection{Processo INAR(1) e sua versão correlacio- nada}

Esta seção será organizada como segue. Daremos alguns resultados preliminares relacionados ao processo INAR(1). Em seguida, introduziremos o modelo INAR(1) correlacionado (CINAR(1)), que inclui o processo INAR(1) como um caso particular. Discutiremos a estimação dos parâmetros e apresentaremos os resultados correspondentes quando a seqüência de inovações possuem distribuição Poisson. Finalmente, ilustraremos o modelo proposto mediante uma aplicação. Os resultados estão publicados em Kolev e Paiva (2000).

\subsubsection{O Processo INAR(1)}

Al-Osh e Alzaid (1987) definem o modelo INAR(1) como

$$
X_{t}=\alpha * X_{t-1}+\epsilon_{t}, \quad t=0,1,2, \ldots,
$$

sendo que $X_{t-1}$ e $\epsilon_{t}$ são independentes, com $X_{t-1} \sim P o(\lambda), \epsilon_{t} \sim P o((1-\alpha) \lambda), \alpha \epsilon$ $[0,1]$. O operador $\alpha * X_{t-1}$ determina a soma aleatória

$$
\alpha * X_{t-1}=\sum_{i=1}^{X_{t-1}} Y_{i}
$$

$\operatorname{com}\left\{Y_{i}\right\}$ sendo uma seqüência de v.a.'s independentes e identicamente distribuídas (i.i.d.) Bernoulli com $\mathbb{P}(Y=1)=1-\mathbb{P}(Y=0)=\alpha$. A v.a. definida em (4.2) 
é uma distribuição de mistura de binomiais e Poisson. O operador mistura $\alpha *$ é conhecido também como binomial thinning, e.g. McKenzie (1988), e foi introduzido por Steutel e van Harn (1979). O operador $\alpha *$ (operador de Steutel e van Harn) substitui a multiplicação escalar no modelo contínuo $\operatorname{AR}(1)$.

O processo (4.1) é estacionário para $\alpha \in(0,1)$. Podemos observar que:

1. se $X_{t} \sim P o(\lambda)$ então $\alpha * X_{t-1} \sim P o(\lambda \alpha)$;

2. se $\epsilon_{t} \sim \operatorname{Po}((1-\alpha) \lambda)$ e independente de $X_{t-1}$, então $X_{t} \sim P o(\lambda)$.

É importante notar que o operador binomial thinning $\alpha *$ é independente do passado histórico do processo. Mais ainda, o processo INAR(1) é Markoviano.

O processo INAR(1) possui as seguintes propriedades (veja Winkelmann (2000))

(i) $0 * X=0, \quad 1 * X=X$;

(ii) $\mathbb{E}(\alpha * X)=\alpha \mathbb{E}(X)$, pois $\mathbb{E}\left(\sum_{i=1}^{X} Y_{i}\right)=\mathbb{E}(X) \mathbb{E}(Y)=\alpha \mathbb{E}(X)$;

(iii) $\underbrace{\alpha * \cdots * \alpha}_{k \text {-vezes }} * X \stackrel{d}{=} \alpha^{k} * X$.

Da equação (4.1) e usando a propriedade (ii), temos que

$$
\mathbb{E}\left(X_{t} \mid X_{t-1}=x_{t-1}\right)=\alpha x_{t-1}+(1-\alpha) \lambda .
$$

Este é um resultado similar ao obtido para o processo AR(1) com inovações normalmente distribuídas, em que a esperança condicional de $X_{t}$ é linear em $x_{t-1}$. Observemos que dado $\epsilon_{t}$ e $x_{t-1}, X_{t}$ é uma v.a. A semelhança com o processo AR(1) não é estendida a momentos de ordem superior. Em particular,

$$
\operatorname{Var}\left(X_{t} \mid X_{t-1}=x_{t-1}\right)=\alpha(1-\alpha) x_{t-1}+(1-\alpha) \lambda
$$


Usando uma substituição recursiva e a propriedade (iii), i.e.,

$$
\begin{aligned}
& X_{t}=\alpha * X_{t-1}+\epsilon_{t}, \\
& X_{t}=\alpha *\left(\alpha * X_{t-2}+\epsilon_{t-1}\right)+\epsilon_{t}, \\
& X_{t}=\alpha *\left(\alpha *\left(\alpha * X_{t-3}+\epsilon_{t-2}\right)+\epsilon_{t-1}\right)+\epsilon_{t},
\end{aligned}
$$

a equação (4.1) pode ser reescrita como

$$
X_{t} \stackrel{d}{=} \alpha^{k} * X_{t-k}+\sum_{j=0}^{k-1} \alpha^{j} * \epsilon_{t-j} .
$$

A distibuição marginal do processo INAR(1) é então dada por

$$
X_{t} \stackrel{d}{=} \sum_{j=0}^{\infty} \alpha^{j} * \epsilon_{t-j} .
$$

O efeito de $\left\{\epsilon_{t}\right\}$ sobre $X_{t}$ é reduzido exponencialmente com o aumento do tamanho dos "lags" usados depois. Assim, a equação (4.3) implica uma estrutura de autocovariância dada por

$$
\begin{aligned}
\gamma(k) & =\operatorname{Cov}\left(X_{t-k}, X_{t}\right)=\operatorname{Cov}\left(X_{t-k}, \alpha^{k} * X_{t-k}\right)+\operatorname{Cov}\left(X_{t-k}, \sum_{j=0}^{k-1} \alpha^{j} * \epsilon_{t-j}\right) \\
& =\alpha^{k} \operatorname{Var}\left(X_{t-k}\right)+\sum_{j=0}^{k-1} \alpha^{j} \operatorname{Cov}\left(X_{t-k}, \epsilon_{t-j}\right)=\alpha^{k} \operatorname{Var}\left(X_{t-k}\right) .
\end{aligned}
$$

As autocorrelações $\rho(k)=\frac{\gamma(k)}{\gamma(0)}$ são restringidas ao intervalo positivo $(0,1)$. A última relação mostra que a função de autocorrelação $\rho(k)$ decai exponencialmente com lag $k$ e tem a mesma forma das equações de Yule-Walker para o modelo AR(1).

No caso particular de $X_{0}$ possuir distribuição Poisson de parâmetro $\lambda$, i.e., $X_{0} \sim \operatorname{Po}(\lambda)$, temos que $\mathbb{E}\left(X_{t}\right)=\lambda$ e $\operatorname{Cov}\left(X_{t}, X_{t-k}\right)=\alpha^{k} \lambda$, para $k=0,1, \ldots, t$ e todo $t$. Para o caso $k=0$, temos $\mathbb{E}\left(X_{t}\right)=\operatorname{Var}\left(X_{t}\right)$. 
Se $\left\{\epsilon_{t}\right\}$ é uma seqüência de v.a.'s i.i.d. não correlacionadas de valores inteiros não negativos, com média $\mu$ e variância finita $\sigma^{2}$, então a média e a variância do processo $X_{t}$ definido por (4.1) são dados por

$$
\mathbb{E}\left(X_{t}\right)=\alpha \mathbb{E}\left(X_{t-1}\right)+\mu=\alpha^{t} \mathbb{E}\left(X_{0}\right)+\mu \sum_{j=0}^{t-1} \alpha^{j}
$$

e

$$
\operatorname{Var}\left(X_{t}\right)=\alpha^{2} \operatorname{Var}\left(X_{t-1}\right)+\alpha(1-\alpha) \mathbb{E}\left(X_{t-1}\right)+\sigma^{2},
$$

i.e.,

$$
\operatorname{Var}\left(X_{t}\right)=\alpha^{2 t} \operatorname{Var}\left(X_{0}\right)+(1-\alpha) \sum_{j=1}^{t} \alpha^{2 j-1} \mathbb{E}\left(X_{t-j}\right)+\sigma^{2} \sum_{j=1}^{t} \alpha^{2(j-1)} .
$$

Observe que a estacionariedade de segunda ordem requer que o valor inicial do processo, $X_{0}$, possua $\mathbb{E}\left(X_{0}\right)=\frac{\mu}{1-\alpha}$ e $\operatorname{Var}\left(X_{0}\right)=\frac{\alpha \mu+\sigma^{2}}{1-\alpha^{2}}$.

O problema de estimação para o processo INAR(1) é mais complicado que para o processo AR(1). Al-Osh e Alzaid (1987) compararam alguns métodos para a estimação dos parâmetros $\alpha$ e $\lambda$ usando estimadores de Yule-Walker, estimadores de máxima verossimilhança condicional e estimadores de máxima verosimilhança.

O mesmo tipo de processo foi introduzido com um nome diferente por McKenzie $(1986,1988)$, o qual derivou várias propriedades para o caso de distribuições marginais específicas (Poisson, geométrica e binomial negativa). 


\subsubsection{Operador de Steutel e van Harn generalizado}

Nesta seção, introduziremos o operador de Steutel e van Harn generalizado $\alpha \bullet$ (veja Stuetel e van Harn (1979)), que estende o conceito do operador $\alpha *$ dado em (4.2).

Seja $\left\{Y_{i}\right\}$ uma seqüência de v.a.'s identicamente distribuídas, positivas, equicorrelacionadas e que assumem valores inteiros, i.e., $P(Y=k)=p_{k}, \quad k=$ $0,1,2, \ldots, \quad \sum_{k=0}^{\infty} p_{k}=1$. Assumamos que $\mathbb{E}(Y)=\alpha<1$ e $\operatorname{Var}(Y)<\infty$, tal que $\operatorname{Corr}\left(Y_{i}, Y_{j}\right)=\rho_{y}$, para $i \neq j$. Definamos o operador $\alpha \bullet_{\rho}$ como a soma aleatória

$$
S_{x} \stackrel{d}{=} \alpha \bullet_{\rho} X \stackrel{d}{=} \sum_{i=1}^{X} Y_{i},
$$

sendo que os $X_{i}$ 's são independentes dos $Y_{i}$ 's.

Com o objetivo de obtermos alguns resultados explícitos para o operador de $r$-ésima ordem $\underbrace{\alpha \bullet_{\rho} \cdots \bullet_{\rho} \alpha}_{r \text {-vezes }} \bullet_{\rho} X \stackrel{d}{=} \alpha^{r} \bullet_{\rho} X$, provaremos o seguinte Lema.

\section{Lema 4.1.}

Sejam $Y_{i}$ 's v.a.'s identicamente distribuídas Bernoulli equicorrelacionadas com média $\alpha$. Temos que a FGP $G_{r}(t)$ da v.a. $\underbrace{\alpha \bullet_{\rho} \cdots \bullet_{\rho} \alpha}_{r-\text { vezes }} \bullet_{\rho} X=\alpha^{r} \bullet X$ pode ser representada por

$G_{r}(t)=1-\left(1-\rho_{Y}+\rho_{Y} \alpha\right)^{r}+\sum_{i=0}^{r}\left(\begin{array}{l}r \\ i\end{array}\right)\left(\alpha \rho_{Y}\right)^{r-i}\left(1-\rho_{Y}\right)^{i} G_{X}\left(1-\alpha^{i}+\alpha^{i} t\right)$.

\section{Prova:}

Provaremos este Lema por indução.

(a) Para $r=1$.

De (2.17), temos que

$$
\alpha \bullet_{\rho} X=\rho_{Y}(1-\alpha)+\rho_{Y} \alpha G_{X}(t)+\left(1-\rho_{Y}\right) G_{X}(1-\alpha+\alpha t), \quad \alpha \in(0,1) .
$$


(b) Assumamos que $\left({ }^{*}\right)$ vale para $n=r$, i.e.,

$\underbrace{\alpha \bullet_{\rho} \cdots \bullet_{\rho} \alpha}_{r \text {-vezes }} \cdot e_{\rho} X=1-\left(1-\rho_{Y}+\rho_{Y} \alpha\right)^{r}+\sum_{i=0}^{r}\left(\begin{array}{l}r \\ i\end{array}\right)\left(1-\rho_{Y}\right)^{i}\left(\alpha \rho_{Y}\right)^{r-i} G_{X}\left(1-\alpha^{i}+\alpha^{i} t\right)$.

(c) Para $n=r+1$, obtemos

$$
\begin{aligned}
& \alpha \bullet{ }_{\rho} \underbrace{\alpha \bullet_{\rho} \cdots \bullet_{\rho} \alpha}_{r \text {-vezes }} \bullet{ }_{\rho} X=\rho_{Y}(1-\alpha)+\rho_{Y} \alpha\left[1-\left(1-\rho_{Y}+\alpha \rho_{Y}\right)^{r}\right. \\
& \left.+\sum_{i=0}^{r}\left(\begin{array}{l}
r \\
i
\end{array}\right)\left(1-\rho_{Y}\right)^{i}\left(\alpha \rho_{Y}\right)^{r-i} G_{X}\left(1-\alpha^{i}+\alpha^{i} t\right)\right] \\
& +\left(1-\rho_{Y}\right)\left[1-\left(1-\rho_{Y}+\alpha \rho_{Y}\right)^{r}\right. \\
& \left.+\sum_{i=0}^{r}\left(\begin{array}{l}
r \\
i
\end{array}\right)\left(1-\rho_{Y}\right)^{i}\left(\alpha \rho_{Y}\right)^{r-i} G_{X}\left(1-\alpha^{i}+\alpha^{i}(1-\alpha+\alpha t)\right)\right] \\
& =1-\left(1-\rho_{Y}+\rho_{Y} \alpha\right)^{r}\left(1-\rho_{Y}+\rho_{Y} \alpha\right) \\
& +\rho_{Y} \alpha \sum_{i=0}^{r}\left(\begin{array}{l}
r \\
i
\end{array}\right)\left(1-\rho_{Y}\right)^{i}\left(\alpha \rho_{Y}\right)^{r-i} G_{X}\left(1-\alpha^{i}+\alpha^{i} t\right) \\
& +\left(1-\rho_{Y}\right) \sum_{i=0}^{r}\left(\begin{array}{l}
r \\
i
\end{array}\right)\left(1-\rho_{Y}\right)^{i}\left(\alpha \rho_{Y}\right)^{r-i} G_{X}\left(1-\alpha^{i}+\alpha^{i}(1-\alpha+\alpha t)\right) \\
& =1-\left(1-\rho_{Y}+\rho_{Y} \alpha\right)^{r+1} \\
& +\sum_{i=0}^{r}\left(\begin{array}{l}
r \\
i
\end{array}\right)\left(1-\rho_{Y}\right)^{i}\left(\alpha \rho_{Y}\right)^{r-i+1} G_{X}\left(1-\alpha^{i}+\alpha^{i} t\right) \\
& +\sum_{i=0}^{r}\left(\begin{array}{l}
r \\
i
\end{array}\right)\left(1-\rho_{Y}\right)^{i+1}\left(\alpha \rho_{Y}\right)^{r-i} G_{X}\left[1-\alpha^{i}+\alpha^{i}(1-\alpha+\alpha t)\right] \\
& =1-\left(1-\rho_{Y}+\rho_{Y} \alpha\right)^{r+1}+\left(\begin{array}{l}
r \\
0
\end{array}\right)\left(\alpha \rho_{Y}\right)^{r+1} G_{X}(t) \\
& +\left(\begin{array}{l}
r \\
1
\end{array}\right)\left(1-\rho_{Y}\right)\left(\alpha \rho_{Y}\right)^{r} G_{X}(1-\alpha+\alpha t) \\
& +\left(\begin{array}{l}
r \\
2
\end{array}\right)\left(1-\rho_{Y}\right)^{2}\left(\alpha \rho_{Y}\right)^{r-1} G_{X}\left(1-\alpha^{2}+\alpha^{2} t\right) \\
& +\ldots+\left(\begin{array}{l}
r \\
r
\end{array}\right)\left(1-\rho_{Y}\right)^{r}\left(\alpha \rho_{Y}\right) G_{X}\left(1-\alpha^{r}+\alpha^{r} t\right) \\
& +\left(\begin{array}{l}
r \\
0
\end{array}\right)\left(1-\rho_{Y}\right)\left(\alpha \rho_{Y}\right)^{r} G_{X}(1-\alpha+\alpha t) \\
& +\left(\begin{array}{l}
r \\
1
\end{array}\right)\left(1-\rho_{Y}\right)^{2}\left(\alpha \rho_{Y}\right)^{r-1} G_{X}\left(1-\alpha^{2}+\alpha^{2} \dot{t}\right)
\end{aligned}
$$




$$
\begin{aligned}
& +\ldots+\left(\begin{array}{c}
r \\
r-1
\end{array}\right)\left(1-\rho_{Y}\right)^{r}\left(\alpha \rho_{Y}\right) G_{X}\left(1-\alpha^{r}+\alpha^{r} t\right) \\
& +\left(\begin{array}{l}
r \\
r
\end{array}\right)\left(1-\rho_{Y}\right)^{r+1}\left(\alpha \rho_{Y}\right)^{0} G_{X}\left(1-\alpha^{r+1}+\alpha^{r+1} t\right) .
\end{aligned}
$$

Por outro lado, para $i=1,2, \ldots, r$, temos que $\left(\begin{array}{c}r+1 \\ i\end{array}\right)=\left(\begin{array}{c}r \\ i\end{array}\right)+\left(\begin{array}{c}r \\ i-1\end{array}\right)$. Então,

$$
\begin{aligned}
\alpha \bullet_{\rho} \underbrace{\alpha \bullet_{\rho} \cdots \bullet_{\rho} \alpha}_{r \text {-vezes }} \cdot \bullet_{\rho} X= & 1-\left(1-\rho_{Y}+\rho_{Y} \alpha\right)^{r+1} \\
& +\sum_{i=0}^{r+1}\left(\begin{array}{c}
k+1 \\
i
\end{array}\right)\left(1-\rho_{Y}\right)^{i}\left(\alpha \rho_{Y}\right)^{r-i+1} G_{X}\left(1-\alpha^{i}+\alpha^{i} t\right) .
\end{aligned}
$$

Desta maneira, fica provado o Lema 4.1.

Esta última expressão é uma mistura de FGP's $G_{X}\left(1-\alpha^{i}+\alpha^{i} t\right)$, para $i=$ $0,1, \ldots, r$ (a soma dos coeficientes é igual a 1 ). As FGP's $G_{X}\left(1-\alpha^{i}+\alpha^{i} t\right)$ representam as somas aleatórias no caso de independência.

Uma interpretação desta última expressão é que a FGP $G_{r}(t)$ é uma composição (com probabilidades correspondentes) das FGP's das somas aleatórias de variáveis aleatórias independentes Bernoulli com parâmetros $\alpha^{i}, i=0,1, \ldots, r$.

\subsubsection{O processo INAR(1) correlacionado}

Agora estamos prontos para definir a extensão do processo INAR(1).

Definição. Uma série de tempo de valores inteiros é chamado um processo CINAR(1), i.e., processo autoregressivo de ordem 1 de valores inteiros correlacionados, se

$$
X_{t} \stackrel{d}{=} \alpha \bullet_{\rho} X_{t-1}+\epsilon_{t}, \quad t=0,1,2 \ldots
$$

sendo que o operador $\alpha \bullet_{\rho}$ é definido em (4.4) e os $Y_{i}$ 's são v.a.'s equicorrelacionadas identicamente distribuídas, $\operatorname{com} \operatorname{Corr}\left(Y_{i}, Y_{j}\right)=\rho_{Y}>0$, para $i \neq j$. 
Os $\epsilon_{t}$ 's são v.a.'s independentes e identicamente distribuídas com média finita $\mu$ e variância $\sigma^{2}$. Além disso, $\epsilon_{t}$ e $X_{t-1}$ são independentes.

O processo CINAR(1) tem uma interpretação intuitiva: $X_{t}$ representa o número de indivíduos de uma população na $t$-ésima geração. Cada indivíduo gera um indivíduo da geração seguinte com probabilidade $\alpha$, sob a suposição de que o processo é equicorrelaconado e não independente cuja quantidade total é um número $\alpha \bullet_{\rho} X_{t}$. Além disso, um número aleatório de indivíduos $\epsilon_{t+1}$ (gerado de uma fonte independente) entra na população de resposta.

Os processos CINAR(1) e INAR(1) possuem propriedades similares. Em particular,

$$
X_{t} \stackrel{d}{=} \alpha^{k} \bullet_{\rho} X_{t-k}+\sum_{j=0}^{k-1} \alpha^{j} \bullet_{\rho} \epsilon_{t-j}
$$

sendo a distribuição marginal do processo CINAR(1) dada por

$$
X_{t} \stackrel{d}{=} \sum_{j=0}^{\infty} \alpha^{j} \bullet_{\rho} \epsilon_{t-j}
$$

A estrutura de autocovariância do processo CINAR(1) é representada por

$$
\begin{aligned}
\gamma(k) & =\operatorname{Cov}\left(X_{t-k}, X_{t}\right)=\alpha^{k} \operatorname{Var}\left(X_{t-k}\right)+\sum_{j=0}^{k-1} \alpha^{j} \operatorname{Cov}\left(X_{t-k}, \epsilon_{t-j}\right) \\
& =\alpha^{k} \operatorname{Var}\left(X_{t-k}\right) .
\end{aligned}
$$

A esperança é dada por

$$
\begin{aligned}
\mathbb{E}\left(X_{t}\right) & =\mathbb{E}\left(X_{t-1}\right) \mathbb{E}(Y)+\mathbb{E}\left(\epsilon_{t}\right)=\alpha \mathbb{E}\left(X_{t-1}\right)+\mu \\
& =\alpha\left[\mathbb{E}\left(X_{t-2}\right) \mathbb{E}(Y)+\mathbb{E}\left(\epsilon_{t-1}\right)\right]+\mu=\alpha^{2} \mathbb{E}\left(X_{t-2}\right)+\mu(\alpha+1) \\
& =\alpha^{2}\left[\mathbb{E}\left(X_{t-3}\right) \mathbb{E}(Y)+\mathbb{E}\left(\epsilon_{t-2}\right)\right]+\alpha \mu+\mu=\alpha^{3} \mathbb{E}\left(X_{t-3}\right)+\mu\left(\alpha^{2}+\alpha+1\right) .
\end{aligned}
$$

Desta maneira, obtemos

$$
\mathbb{E}\left(X_{t}\right)=\alpha^{t} \mathbb{E}\left(X_{0}\right)+\mu \sum_{j=0}^{t-1} \alpha^{j},
$$


A variância do processo CINAR(1) é dada por

$$
\begin{aligned}
\operatorname{Var}\left(X_{t}\right)= & \operatorname{Var}\left(X_{t-1}\right) \mathbb{E}^{2}(Y)+\mathbb{E}\left(X_{t-1}\right) \operatorname{Var}(Y) \\
& +\rho \operatorname{Var}(Y) \mathbb{E}\left[X_{t-1}\left(X_{t-1}-1\right)\right]+\operatorname{Var}\left(\epsilon_{t}\right) \\
= & \operatorname{Var}\left(X_{t-2}\right) \mathbb{E}^{4}(Y)+\mathbb{E}\left(X_{t-2}\right) \operatorname{Var}(Y) \mathbb{E}^{2}(Y) \\
& +\rho \operatorname{Var}(Y) \mathbb{E}^{2}(Y) \mathbb{E}\left[X_{t-2}\left(X_{t-2}-1\right)\right]+\mathbb{E}^{2}(Y) \operatorname{Var}\left(\epsilon_{t-1}\right) \\
& +\mathbb{E}\left(X_{t-1}\right) \operatorname{Var}(Y)+\rho \operatorname{Var}(Y) \mathbb{E}\left[X_{t-1}\left(X_{t-1}-1\right)\right]+\operatorname{Var}\left(\epsilon_{t}\right) \\
= & \operatorname{Var}\left(X_{t-3}\right) \mathbb{E}^{6}(Y)+\mathbb{E}\left(X_{t-3}\right) \operatorname{Var}(Y) \mathbb{E}^{4}(Y) \\
& +\mathbb{E}\left(X_{t-2}\right) \operatorname{Var}(Y) \mathbb{E}^{2}(Y) \mathbb{E}\left(X_{t-1}\right) \operatorname{Var}(Y) \\
& +\rho \operatorname{Var}(Y) \mathbb{E}^{4}(Y) \mathbb{E}\left[X_{t-3}\left(X_{t-3}-1\right)\right] \\
& +\rho \operatorname{Var}(Y) \mathbb{E}^{2}(Y) \mathbb{E}\left[X_{t-2}\left(X_{t-2}-1\right)\right]+\rho \operatorname{Var}(Y) \mathbb{E}\left[X_{t-1}\left(X_{t-1}-1\right)\right] \\
& +\mathbb{E}^{4}(Y) \operatorname{Var}\left(\epsilon_{t-2}\right)+\mathbb{E}^{2}(Y) \operatorname{Var}\left(\epsilon_{t-1}\right)+\operatorname{Var}\left(\epsilon_{t}\right) .
\end{aligned}
$$

Desta maneira, obtemos

$$
\begin{aligned}
\operatorname{Var}\left(X_{t}\right)= & \alpha^{2 t} \operatorname{Var}\left(X_{0}\right)+(1-\alpha) \sum_{j=1}^{t} \alpha^{2 j-1}\left\{E\left(X_{t-j}\right)+\rho E\left[X_{t-j}\left(X_{t-j}-1\right)\right]\right\} \\
& +\sigma^{2} \sum_{j=1}^{t} \alpha^{2(j-1)} .
\end{aligned}
$$

A estacionariedade de segunda ordem no processo CINAR(1) não pode ser obtida como no caso do processo $\operatorname{INAR}(1)$, pois o processo $\operatorname{CINAR}(1)$ não é estacionário, como foi considerado antes em processos autoregressivos de primeira ordem.

\subsubsection{Estimação dos parâmetros}

A estimação dos parâmetros do processo CINAR(1) pode ser feita pelo método de máxima verossimilhança. O operador Binomial Thinning correlacionado em 
(4.5) é independente do passado histórico do processo. Assim, o modelo CINAR(1) tem propriedades Markovianas

$$
\mathbb{P}\left(X_{t} \mid x_{t-1}, x_{t-2}, \ldots\right)=\mathbb{P}\left(X_{t} \mid x_{t-1}\right)
$$

e a distribuição conjunta pode ser calculada como

$$
\mathbb{P}\left(X_{t}, X_{t-1}, X_{t-2}, \ldots\right)=\mathbb{P}\left(X_{t} \mid x_{t-1}\right) \mathbb{P}\left(X_{t-1} \mid x_{t-2}\right) \ldots \mathbb{P}\left(X_{0}\right)
$$

A função de verossimilhança para a amostra $\mathrm{x}=\left(x_{0}, x_{1}, \ldots, x_{N}\right)$ é

$$
L_{N}(\mathrm{x}, \theta)=\mathbb{P}_{\theta}\left(X_{0}=x_{0}\right) \prod_{t=1}^{N} \mathbb{P}_{\theta}\left(x_{t-1}, x_{t}\right),
$$

sendo que $\theta \in \Theta$ é o vetor de parâmetros desconhecidos e $\mathbb{P}_{\theta}\left(x_{t-1}, x_{t}\right)$ são as correspondentes probabilidades de transição da cadeia de Markov.

Desconsideremos a dependência do valor inicial e consideremos como um estimador para $\theta$ o estimador de máxima verossimilhança condicional dado $X_{0}=x_{0}$, que conseguimos maximizando a função de log-verossimilhança condicional sobre $\Theta:$

$$
\log L_{N}(\mathrm{x}, \theta)=l_{N}\left(\mathrm{x}, \theta \mid x_{0}\right)=\sum_{t=1}^{N} \log \mathbb{P}_{\theta}\left(x_{t-1}, x_{t}\right)
$$

Apliquemos este método para o processo CINAR(1)

$$
X_{t} \stackrel{d}{=} \alpha \bullet_{\rho} X_{t-1}+\epsilon_{t}, \quad t=0,1,2 \ldots
$$

com inovações $\epsilon_{t} \sim \operatorname{Po}(\lambda)$. Assim, o vetor de parâmetros desconhecidos será $\theta=$ $(\alpha, \rho, \lambda)$, sendo $\alpha$ a probabilidade de sobrevivência de um indivíduo, $\rho$ o coeficiente de correlação entre os indivíduos e $\lambda$ o número médio (intensidade) de indivíduos que entram no final de cada período $(t-1, t]$, i.e., no momento $t, t=1,2 \ldots$ 
A distribuição de $X_{t}$ dado $x_{t-1}$ é uma mistura de binomiais correlacionadas e Poisson, calculada da maneira seguinte

$$
\begin{aligned}
\mathbb{P}_{\theta}\left(x_{t-1}, x_{t}\right)= & \mathbb{P}\left(X_{t}=n \mid x_{t-1}=m\right) \\
= & \mathbb{P}\left(\sum_{j=1}^{X_{t-1}} Y_{j}+\epsilon_{t}=n \mid X_{t-1}=m\right) \\
= & \sum_{j=1}^{m} \mathbb{P}\left(V=j, \epsilon_{t}=n-j \mid x_{t-1}=j\right) \\
= & \mathbb{P}(V=0) \mathbb{P}\left(\epsilon_{t}=n\right)+\sum_{j=1}^{m-1} \mathbb{P}(V=j) \mathbb{P}\left(\epsilon_{t}=n-j\right) \\
& +\mathbb{P}(V=m) \mathbb{P}\left(\epsilon_{t}=n-m\right),
\end{aligned}
$$

sendo que $V=\sum_{j=1}^{X_{t-1}} Y_{j}$ tem distribuição dada por (2.19). Temos então

$$
\begin{aligned}
\mathbb{P}_{\theta}\left(x_{t-1}, x_{t}\right)= & {\left[(1-\rho)(1-\alpha)^{m}+\rho(1-\alpha)\right] \frac{e^{-\lambda} \lambda^{n}}{n !} } \\
& +\sum_{j=1}^{m-1}\left[(1-\rho)\left(\begin{array}{c}
m \\
j
\end{array}\right) \alpha^{j}(1-\alpha)^{m-j}\right] \frac{e^{-\lambda} \lambda^{n-j}}{(n-j) !} \\
& +\left[(1-\rho) \alpha^{m}+\rho \alpha\right] \frac{e^{-\lambda} \lambda^{n-m}}{(n-m) !} .
\end{aligned}
$$

Portanto, a distribuição de $X_{t}$ dado $x_{t-1}$ pode ser representada por

$$
\begin{aligned}
\mathbb{P}_{\theta}\left(x_{t-1}, x_{t}\right)= & (1-\rho) e^{-\lambda} \sum_{j=0}^{\min \{m, n\}} \frac{\lambda^{n-j}}{(n-j) !}\left(\begin{array}{c}
m \\
j
\end{array}\right) \alpha^{j}(1-\alpha)^{m-j} \\
& +\rho e^{-\lambda} \frac{\lambda^{n}}{n !}(1-\alpha)+\rho e^{-\lambda} \frac{\lambda^{n-m}}{(n-m) !} \alpha \mathbf{1}\{n \geq m\},
\end{aligned}
$$

sendo que $1\{$.$\} é a função indicadora.$

Observemos que se $\rho=0$, temos a relação válida para o processo $\operatorname{INAR}(1)$, veja Al-Osh e Alzaid (1987).

Denotemos por $\hat{\theta}=(\hat{\alpha}, \hat{\rho}, \hat{\lambda})$ o estimador de máxima verossimilhança condicio- 
nal, sendo ele obtido como solução do sistema de equações

$$
\frac{\partial l_{N}}{\partial \alpha}=0, \quad \frac{\partial l_{N}}{\partial \rho}=0 \quad \text { e } \quad \frac{\partial l_{N}}{\partial \lambda}=0 .
$$

Por exemplo,

$$
\begin{aligned}
\frac{\partial l_{N}}{\partial \alpha} & =\frac{\partial}{\partial \alpha} \sum_{t=1}^{N} \log \mathbb{P}_{\theta}\left(x_{t-1}, x_{t}\right)=\sum_{t=1}^{N} \frac{\partial}{\partial \alpha} \log \mathbb{P}_{\theta}\left(x_{t-1}, x_{t}\right) \\
& =\sum_{t=1}^{N}\left[\frac{1}{\mathbb{P}_{\theta}} \frac{\partial \mathbb{P}_{\theta}}{\partial \alpha}\right]
\end{aligned}
$$

sendo que

$$
\begin{aligned}
\frac{\partial \mathbb{P}_{\theta}}{\partial \alpha}= & (1-\rho) e^{-\lambda} \sum_{j=0}^{\min \left\{x_{t-1}, x_{t}\right\}} \frac{\lambda^{x_{t}-j}}{\left(x_{t}-j\right) !}\left(\begin{array}{c}
x_{t-1} \\
j
\end{array}\right)\left[\alpha^{j-1}(1-\alpha)^{x_{t-1}-j-1}\left(j-\alpha x_{t-1}\right)\right] \\
& -\rho e^{-\lambda} \frac{\lambda^{x_{t}}}{x_{t} !}+\rho e^{-\lambda} \frac{\lambda^{x_{t}-x_{t-1}}}{\left(x_{t}-x_{t-1}\right) !} \mathbf{1}\left\{x_{t} \geq x_{t-1}\right\},
\end{aligned}
$$

e $\mathbb{P}_{\theta}$ é dado em (4.7). De maneira análoga, são encontradas as outras derivadas (veja Apêndice A).

Os estimadores de máxima verossimilhança condicional $\hat{\theta}=(\hat{\alpha}, \hat{\rho}, \hat{\lambda})$ dado $x_{0}$ foram obtidos usando o método de Newton-Raphson.

Os valores iniciais dos parâmetros $\alpha$ e $\lambda$ foram obtidos pelo procedimento de estimação baseado na minimização da soma de quadrados dos desvios sobre a esperança condicional, desenvolvido por Klimko e Nelson (1978).

Os estimadores de mínimos quadrados condicional para $\alpha$ e $\lambda$ são aqueles valores que minimizam

$$
F\left(\alpha, \lambda, x_{t-1}\right)=\sum_{t=1}^{n}\left[X_{t}-\mathbb{E}\left(X_{t} \mid x_{t-1}\right)\right]^{2} .
$$

Para a média condicional do processo CINAR(1) com inovações Poisson temos

$$
\mathbb{E}\left(X_{t} \mid X_{t-1}=x_{t-1}\right)=\alpha x_{t-1}+\lambda .
$$


Na avaliação das derivadas da última expressão com respeito a $\alpha$ e $\lambda$ obtemos

$$
\hat{\alpha}=\frac{\sum_{t=1}^{n} x_{t} x_{t-1}-\frac{1}{n} \sum_{t=1}^{n} x_{t} \sum_{t=1}^{n} x_{t-1}}{\sum_{t=1}^{n} x_{t-1}^{2}-\frac{1}{n}\left(\sum_{t=1}^{n} x_{t-1}\right)^{2}},
$$

e

$$
\hat{\lambda}=\frac{1}{n} \sum_{t=1}^{n} x_{t}-\frac{1}{n} \hat{\alpha} \sum_{t=1}^{n} x_{t}
$$

Pode-se verificar que as condições necessárias de regularidade do Teorema 3.2 de Klimko e Nelson (1978) são satisfeitas e que $n^{\frac{1}{2}}\left(\hat{\theta}-\hat{\theta}^{0}\right)$ é assintoticamente normalmente distribuídas em que $\hat{\theta}^{0}=\left(\alpha^{0}, \rho^{0}, \lambda^{0}\right)$ denota os valores "verdadeiros" dos parâmetros.

Uma outra forma mais simples para conseguir um estimador inicial para $\alpha$ é substituir $\gamma(k)$ com a função de autocovariância na equação de Yule-Walker (4.6). Assim, obtemos

$$
\hat{\alpha}=\frac{\sum_{t=0}^{n-1}\left(x_{t}-\bar{x}\right)\left(x_{t+1}-\bar{x}\right)}{\sum_{t=0}^{n-1}\left(x_{t}-\bar{x}\right)^{2}},
$$

em que $\bar{x}$ é a média amostral.

\subsubsection{Ilustração}

Os dados apresentados nesta ilustração correspondem ao número diário de pacientes com deficiência respiratória do PROAIM (Programa de Aprimoramento das Informações de Mortalidade no Município de São Paulo) durante o período de 1996 a 1997. Apesar deles não serem apropriados para ajustar os processos INAR(1) e CINAR(1), o fizemos assumindo inovações Poisson e obtivemos as respectivas estimativas dos parâmetros.

Em seguida, apresentamos os gráficos das séries originais, das séries dos resíduos e das funções de autocorrelação dos resíduos para os dois modelos. 
Grafico da Serie

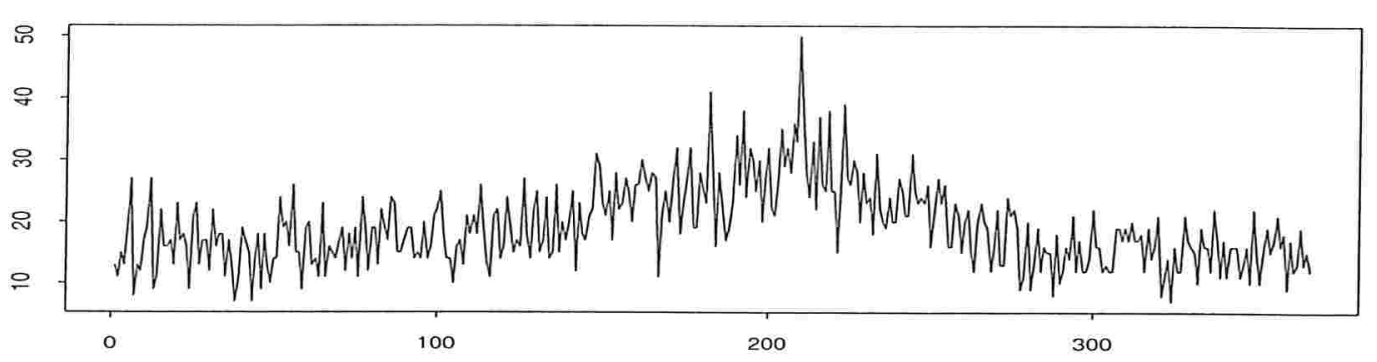

Grafico dos Residuos

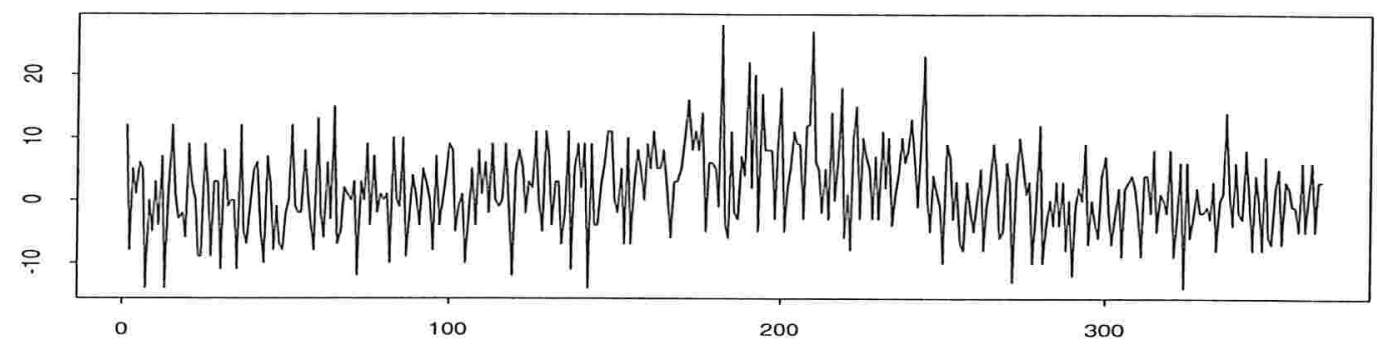

Funcao de Autocorrelacao dos Residuos

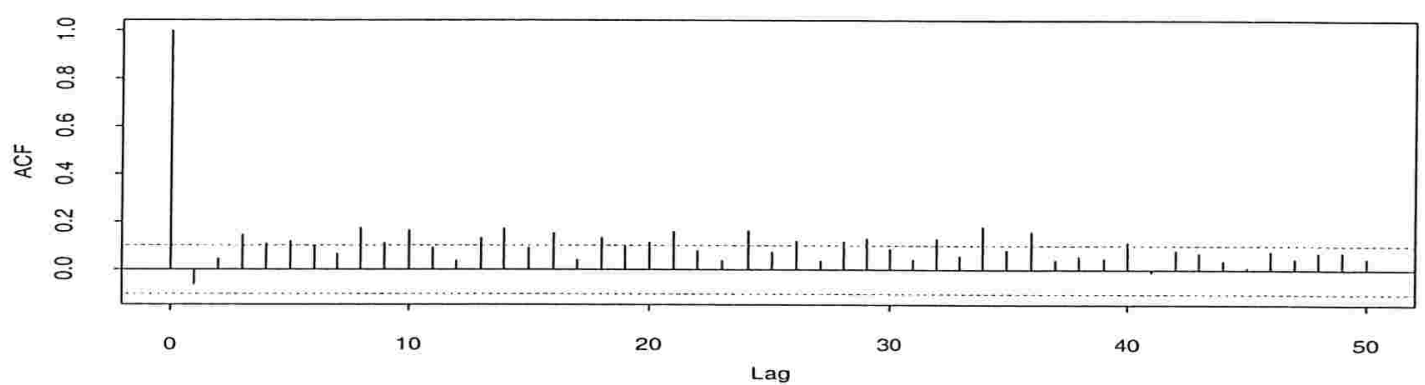

Figura 4.1: Gráficos da série, dos resíduos e da função de autocorrelação dos resíduos para o modelo $I N A R(1)$ no período de 1996. 
Grafico da Serie

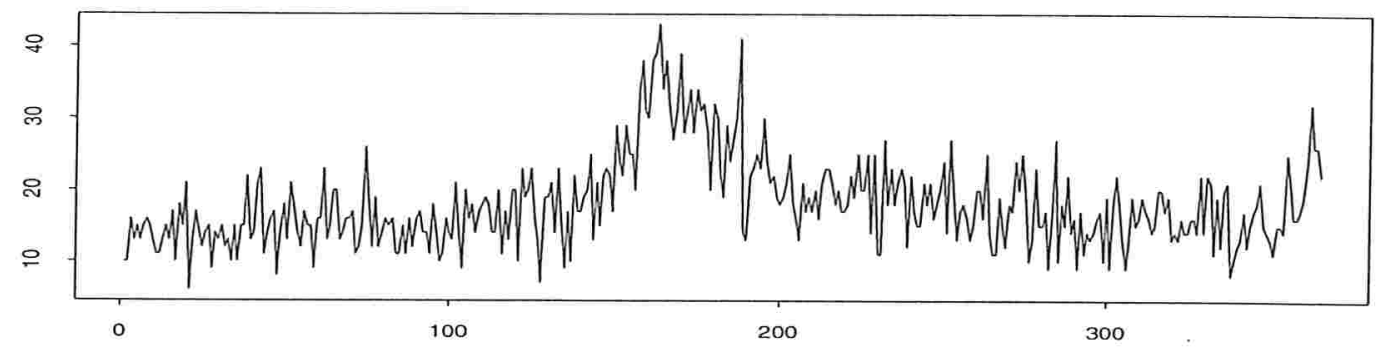

Grafico dos Residuos

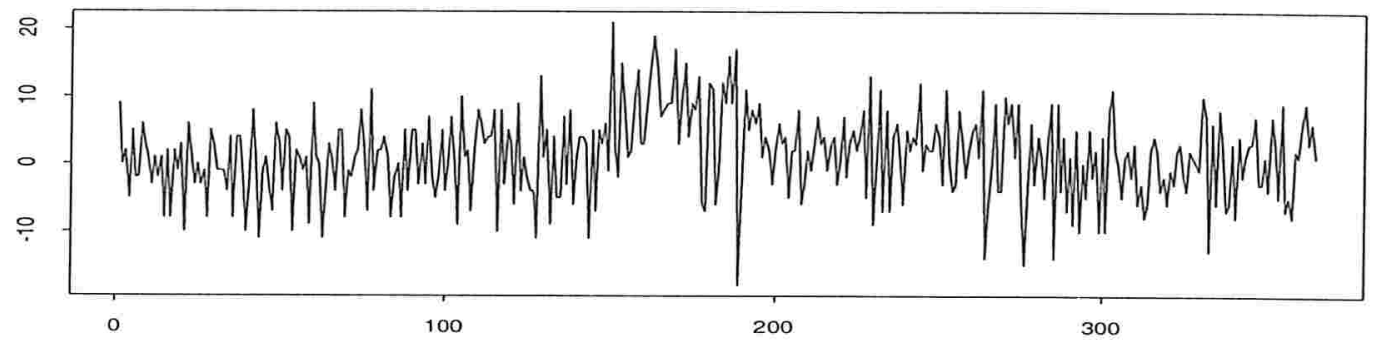

Funcao de Autocorrelacao dos Residuos

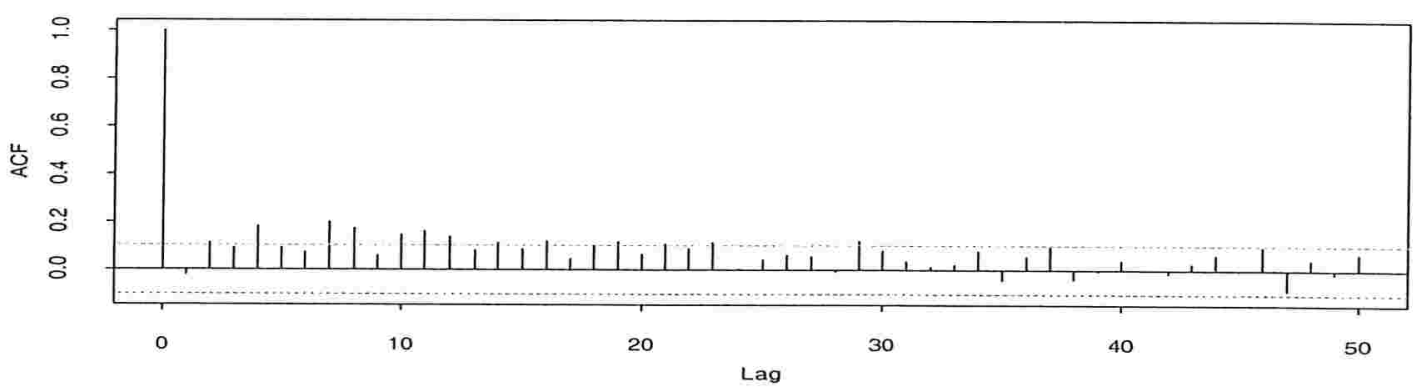

Figura 4.2: Gráficos da série, dos resíduos e da função de autocorrelação dos residuos, para o modelo INAR(1) no período de 1997. 
Grafico da Serie

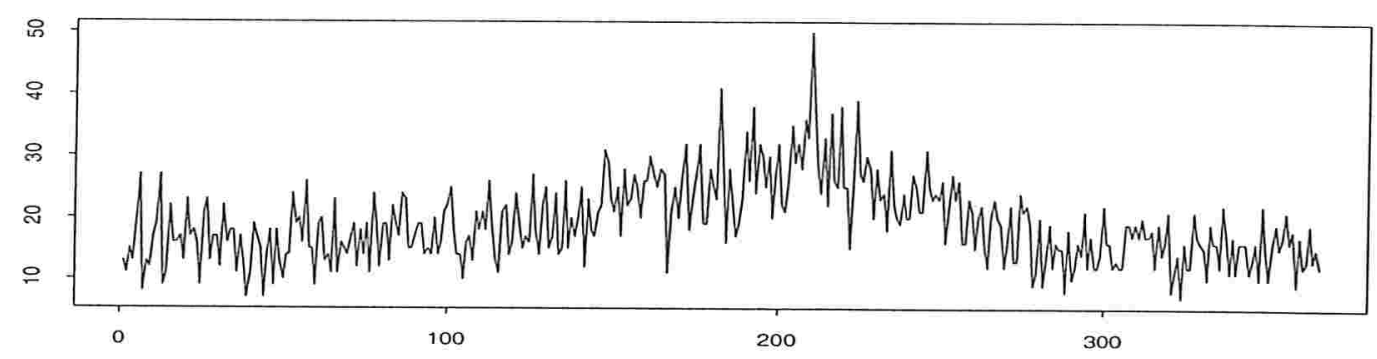

Grafico dos Residuos

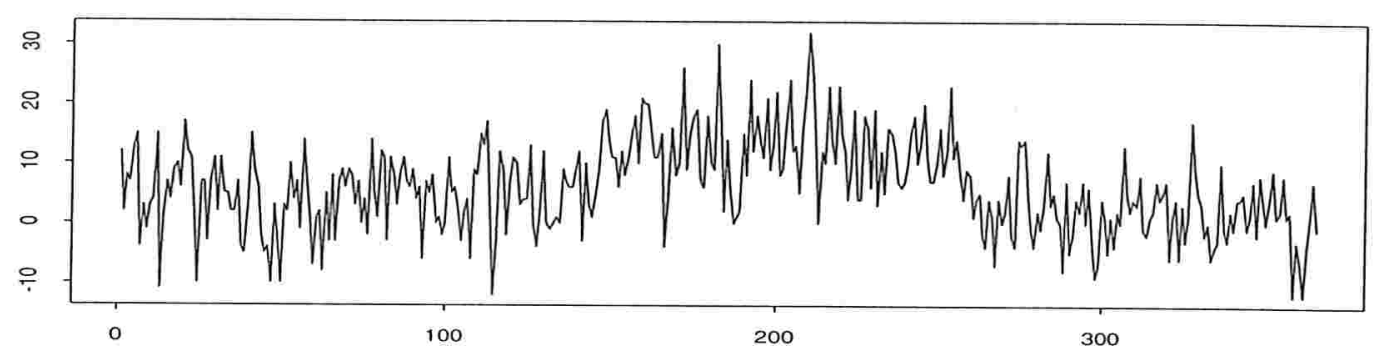

Funcao de Autocorrelacao dos Residuos

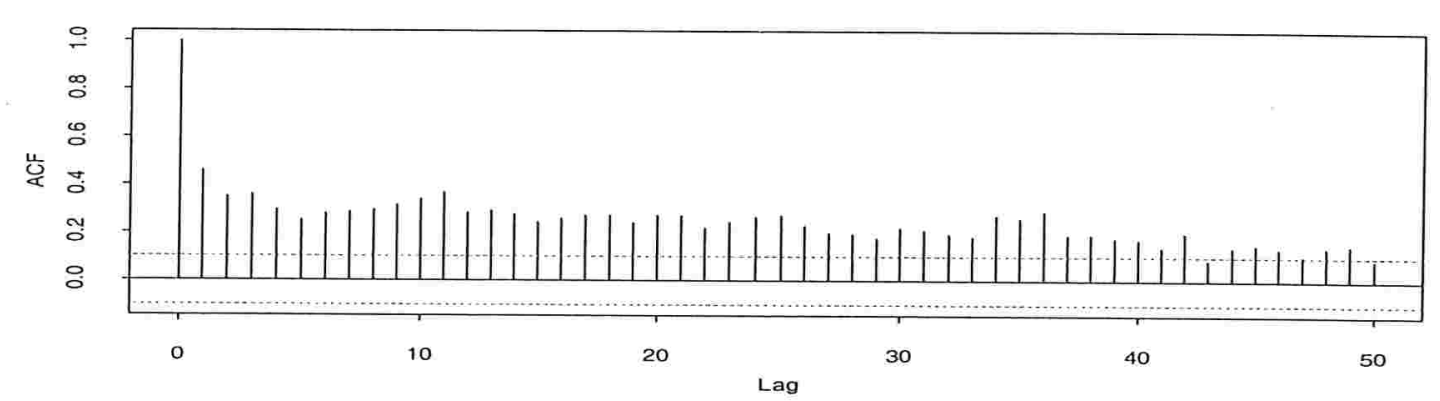

Figura 4.3: Gráficos da série, dos resíduos e da função de autocorrelação dos resíduos, para o modelo CINAR(1) no periodo de 1996. 
Grafico da Serie

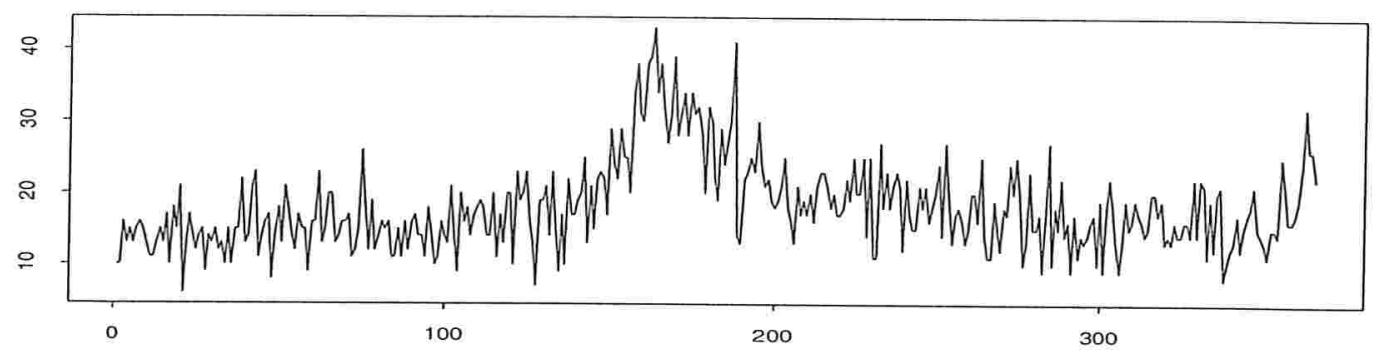

Grafico dos Residuos

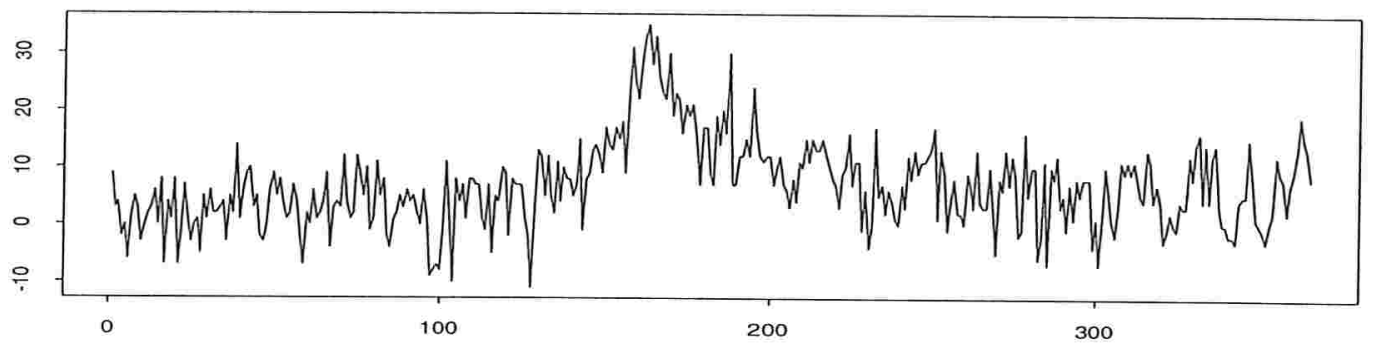

Funcao de Autocorrelacao dos Residuos

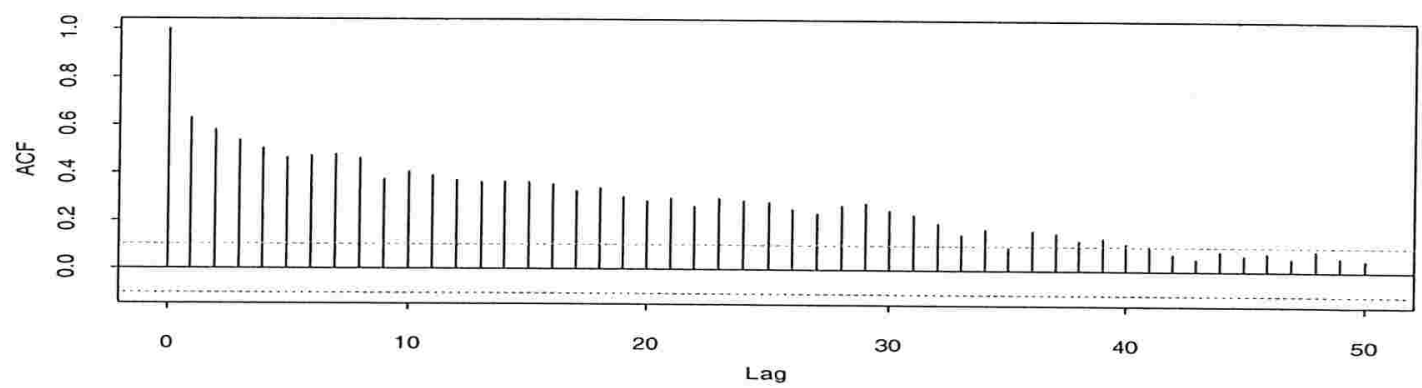

Figura 4.4: Gráficos da série, dos resíduos e da função de autocorrelação dos resíduos, para o modelo CINAR(1) no período de $199 \%$.

Nas Figuras 4.1 e 4.2, foram apresentados os gráficos da série, dos resíduos e as funções de autocorrelação dos modelos INAR(1) para os anos de 1996 e de 1997 , respectivamente. Observemos que a série original apresenta variação de nível em torno do dia 200 e também de variabilidade. Os gráficos dos resíduos mostram variação em torno de zero com alguns valores altos por volta do dia 200. As funções de 
autocorrelação dos resíduos nos indicam que o comportamento é aproximadamente um ruído branco.

Os gráficos da série, dos resíduos e as funções de autocorrelação para os modelos CINAR(1) nos períodos de 1996 e de 1997 estão nas Figuras 4.3 e 4.4, respectivamente. Podemos observar que a série mostra também variação de nível e de variabilidade em torno do dia 200 e as séries dos resíduos continuam apresentando variação de nível e de variabilidade. As funções de autocorrelação dos resíduos não decaem rapidamente para zero, indicando a presença de não estacionariedade na série.

Os valores iniciais dos parâmetros $\alpha_{0}$ e $\lambda_{0}$ foram calculados de acordo com as fórmulas (4.8) e (4.9) e são dados na Tabela 4.1.

Tabela 4.1: Parâmetros estimados dos modelos CINAR(1) e INAR(1) para os dados de doença respiratória nos períodos de 1996 e $199 \%$.

\begin{tabular}{ccccccccc}
\hline & \multicolumn{3}{c}{ Valores iniciais } & \multicolumn{3}{c}{$\operatorname{CINAR}(1)$} & \multicolumn{3}{c}{$\operatorname{INAR}(1)$} \\
\hline \multirow{2}{*}{ ano } & $\alpha_{0}$ & $\rho_{0}$ & $\lambda_{0}$ & $\hat{\alpha}$ & $\hat{\rho}$ & $\hat{\lambda}$ & $\hat{\alpha}$ & $\hat{\lambda}$ \\
\hline \multirow{2}{*}{1996} & 0,50 & 0,99 & 9,49 & 0,46 & 0,27 & 6,98 & 0,48 & 7,94 \\
& & & & $(0,06)$ & $(0,02)$ & $(0,18)$ & $(0,13)$ & $(0,29)$ \\
\multirow{2}{*}{1997} & 0,58 & 0,99 & 7,62 & 0,55 & 0,15 & 5,34 & 0,54 & 6,72 \\
& & & & $(0,08)$ & $(0,11)$ & $(0,21)$ & $(0,17)$ & $(0,19)$ \\
\hline
\end{tabular}

O valor inicial do parâmetro $\rho_{0}$ do modelo CINAR(1) é encontrado usando os valores já encontrados de $\alpha_{0}$ e $\lambda_{0}$. Estes valores $\left(\alpha_{0}, \rho_{0}\right.$ e $\left.\lambda_{0}\right)$ foram usados como valores iniciais para o algoritmo de Newton-Raphson. As estimativas ótimas e os valores dos desvios padrão são também dados na. Tabela 4.1. Estas estimativas foram obtidas usando a linguagem de programação OX (ver Doornik, 1996). No Apêndice B, encontram-se os programas utilizados. 
De acordo com o modelo CINAR(1), $\alpha$ é a probabilidade de sobrevivência de um indivíduo. Num ano, esta probabilidade cresce 0,09. Isto indica algum incremento significativo na média de poluição do ar em São Paulo sobre o mesmo período de tempo, o qual é confirmado por estudos anteriores. O número médio de pacientes que entraram diariamente no PROAIM (representado pelo parâmetro ג) durante os anos de 1996 e de 1997 é 6,98 e 5,34, respectivamente. Este é um resultado surpreendente, pois a maior poluição no ar intuitivamente deveria causar um maior número de pacientes com deficiência respiratória.

Conclusões similares podem ser obtidas por meio da interpretação detalhada dos parâmetros $\alpha$ e $\lambda$ do processo INAR(1).

Na Tabela 4.1, observemos que o valor do estimador $\rho$ indica que existe dependência entre as variáveis binárias $Y_{i}$ 's envolvidas na construção do processo CINAR(1). O valor estimado de $\rho$ para o ano de 1997 é menor do que para o ano de 1996. Isto mostra que a correlação entre a morte dos pacientes decresce quando a poluição do ar também decresce.

Para a escolha do modelo, usamos os critérios AIC (Akaike Information Criterion) e BIC (Bayes Information Criterion), veja Shumway (2000). Obtivemos os seguintes resultados 
Tabela 4.2: Valores dos coeficientes $A I C$ e BIC para os modelos INAR(1) e CINAR(1) nos periodos de 1996 e 1997.

\begin{tabular}{ccccccc}
\hline Ano & Modelo & $\hat{\sigma_{a}^{2}}$ & $\begin{array}{c}\text { Número de } \\
\text { parâmetros }\end{array}$ & $\begin{array}{c}\text { Tamanho da } \\
\text { amostra }\end{array}$ & AIC & BIC \\
\hline \multirow{2}{*}{1996} & INAR(1) & 48,69 & 2 & 366 & 3,89 & 3,92 \\
& CINAR(1) & 56,52 & 3 & 366 & 4,05 & 4,09 \\
\multirow{2}{*}{1997} & INAR(1) & 38,83 & 2 & 365 & 3,67 & 3,69 \\
& CINAR(1) & 56,42 & 3 & 365 & 4,06 & 4,08 \\
\hline
\end{tabular}

Observemos que, para o modelo CINAR(1), os valores dos critérios AIC e BIC e da variância residual $\left(\sigma_{a}^{2}\right)$ nos anos de 1996 e 1997 são muito próximos, o mesmo não ocorrendo para o modelo INAR(1).

\subsection{Duas extensões do processo DAR(1)}

Nesta seção, estudaremos o processo autoregressivo de ordem 1 discreto, i.e., $\operatorname{DAR}(1)$, que foi introduzido por Jacobs e Lewis (1978), como segue:

$$
X_{t}=U_{t} X_{t-1}+\left(1-U_{t}\right) \epsilon_{t}, \quad t=2, \ldots, m
$$

A seqüência $\left\{U_{t}\right\}$ é composta por v.a.'s independentes Bernoulli com $P\left(U_{t}=\right.$ $0)=1-P\left(U_{t}=1\right)=1-r, 0<r<1$, e $\left\{\epsilon_{t}\right\}$ sendo uma seqüência de v.a.'s discretas e i.i.d. Para cada $t$, as inovações $\epsilon_{t}$ são independentes de $X_{t-1}$ e $U_{t}$. É fácil mostrar que (4.10) é um processo Markoviano estacionário.

Nesta seção, sugeriremos duas extensões do processo DAR(1). A primeira será baseada no operador binomial lninning, definido em (4.2), e a segunda será obtida 
por meio de sua versão correlacionada; veja (4.4). O propósito deste capítulo é sugerir modelos de dependência mais flexíveis para processos de contagem. Apresentaremos também a estimação de máxima verossimilhança condicional dos parâmetros dos modelos no caso de inovações Poisson. Os resultados desta seção estão publicados em Paiva e Kolev (2001b).

\subsubsection{Processo DAR(1) com Binomial thinning}

Consideremos a seguinte modificação de (4.10). No lugar de $X_{t-1}$ em (4.10), usemos o operador binomial thinning $\alpha * X_{t-1}$ já definido. Assim, esse primeiro modelo pode ser descrito da seguinte forma:

$$
\left\{\begin{array}{l}
X_{1}=\varepsilon_{1} \\
X_{t}=U_{t}\left(\alpha * X_{t-1}\right)+\left(1-U_{t}\right) \varepsilon_{t}, \quad 2 \leq t \leq m
\end{array}\right.
$$

Usando uma substituição recursiva e a propriedade $\underbrace{\alpha * \ldots * \alpha}_{k-\text { vezes }} * X \stackrel{d}{=} \alpha^{k} * X$, para $k=1,2, \ldots, t$, o processo (4.11) pode ser descrito por

$$
X_{t}=\left(1-U_{t}\right) \varepsilon_{t}+\sum_{j=1}^{k-1}\left[\left(1-U_{t-j}\right)\left(\alpha^{j} * \varepsilon_{t-j}\right) \prod_{i=0}^{j-1} U_{t-i}\right]+\left(\alpha^{k} * X_{t-k}\right) \prod_{i=0}^{k-1} U_{t-i} .
$$

A estrutura de auto-covariância do processo (4.11) é dada por

$$
\begin{aligned}
\gamma(k) & =\operatorname{Cov}\left(X_{t-k} ; X_{t}\right)=\operatorname{Cov}\left(X_{t-k} ;\left(\alpha^{k} * X_{t-k}\right) \prod_{i=0}^{k-1} U_{t-i}\right) \\
& =\mathbb{E}\left[X_{t-k}\left(\alpha^{k} * X_{t-k}\right) \prod_{i=0}^{k-1} U_{t-i}\right]-\mathbb{E}\left[X_{t-k}\right] \mathbb{E}\left[\left(\alpha^{k} * X_{t-k}\right) \prod_{i=0}^{k-1} U_{t-i}\right] \\
& =r^{k} \mathbb{E}\left[X_{t-k}\left(\alpha^{k} * X_{t-k}\right)\right]-\mathbb{E}\left[X_{t-k}\right] \alpha^{k} \mathbb{E}\left[X_{t-k}\right] r^{k} \\
& =(\alpha r)^{k} \mathbb{E}\left[X_{t-k}^{2}\right]-(\alpha r)^{k} \mathbb{E}^{2}\left[X_{t-k}\right] \\
& =(\alpha r)^{k} \operatorname{Var}\left(X_{t-k}\right) .
\end{aligned}
$$


Para garantir a estacionariedade de primeira ordem de (4.11), consideremos inovações Poisson com parâmetro $\frac{\lambda(1-r \alpha)}{1-r}, \lambda>0$, i.e., $\epsilon_{t} \sim P o\left(\frac{\lambda(1-r \alpha)}{1-r}\right)$. Neste caso, a. esperança é dada por

$$
\begin{aligned}
\mathbb{E}\left(X_{t}\right) & =\lambda(1-\alpha r)+\lambda(1-\alpha r) \sum_{j=1}^{k-1}(\alpha r)^{j}+\alpha^{k} \lambda r^{k} \\
& =\lambda(1-\alpha r) \sum_{j=0}^{k-1}(\alpha r)^{j}+\lambda(\alpha r)^{k} \\
& =\lambda \sum_{j=0}^{k-1}(\alpha r)^{j}-\lambda \sum_{j=1}^{k-1}(\alpha r)^{j}=\lambda .
\end{aligned}
$$

A variância do processo (4.11) pode ser calculada da seguinte forma. Para facilitar a notação, consideremos $\beta_{t}=\left(1-U_{t}\right) \varepsilon_{t}$. Assim,

$$
\begin{aligned}
\operatorname{Var}\left(X_{t}\right)= & \operatorname{Var}\left[\beta_{t}+U_{t}\left(\alpha * X_{t-1}\right)\right] \\
= & \operatorname{Var}\left(\beta_{t}\right)+\mathbb{E}\left(U_{t}^{2}\right) \mathbb{E}\left[\left(\alpha * X_{t-1}\right)^{2}\right]-\mathbb{E}^{2}\left(U_{t}\right) \mathbb{E}^{2}\left(\alpha * X_{t-1}\right) \\
= & \operatorname{Var}\left(\beta_{t}\right)+\mathbb{E}\left(U_{t}^{2}\right)\left[\alpha^{2} \operatorname{Var}\left(X_{t-1}\right)+\alpha(1-\alpha) \mathbb{E}\left(X_{t-1}\right)\right] \\
& +\operatorname{Var}\left(U_{t}\right) \mathbb{E}^{2}\left(\alpha * X_{t-1}\right) \\
= & \operatorname{Var}\left(\beta_{t}\right)+\alpha^{2} \mathbb{E}\left(U_{t}^{2}\right) \operatorname{Var}\left(X_{t-1}\right)+\alpha(1-\alpha) \mathbb{E}\left(U_{t}^{2}\right) \mathbb{E}\left(X_{t-1}\right) \\
& +\alpha^{2} \operatorname{Var}\left(U_{t}\right) \mathbb{E}^{2}\left(X_{t-1}\right) \\
= & \operatorname{Var}\left(\beta_{t}\right)+\alpha^{2} \mathbb{E}\left(U_{t}^{2}\right) \operatorname{Var}\left(\beta_{t-1}\right)+\alpha^{4} \mathbb{E}\left(U_{t}^{2}\right) \mathbb{E}\left(U_{t-1}^{2}\right) \operatorname{Var}\left(X_{t-2}\right) \\
& +\alpha^{3}(1-\alpha) \mathbb{E}\left(U_{t}^{2}\right) \mathbb{E}\left(U_{t-1}^{2}\right) \mathbb{E}\left(X_{t-2}\right)+\alpha(1-\alpha) \mathbb{E}\left(U_{t}^{2}\right) \mathbb{E}\left(X_{t-1}\right) \\
= & \operatorname{Var}\left(\beta_{t}\right)+\alpha^{2} \mathbb{E}\left(U_{t}^{2}\right) \operatorname{Var}\left(\beta_{t-1}\right)+\alpha^{4} \mathbb{E}\left(U_{t}^{2}\right) \mathbb{E}\left(U_{t-1}^{2}\right) \operatorname{Var}\left(\beta_{t-2}\right) \\
& +\alpha^{6} \mathbb{E}\left(U_{t}^{2}\right) \mathbb{E}\left(U_{t-1}^{2}\right) \mathbb{E}\left(U_{t-2}^{2}\right) \operatorname{Var}\left(X_{t-3}\right) \\
& +\alpha^{5}(1-\alpha) \mathbb{E}\left(U_{t}^{2}\right) \mathbb{E}\left(U_{t-1}^{2}\right) \mathbb{E}\left(U_{t-2}^{2}\right) \mathbb{E}\left(X_{t-3}\right) \\
& +\alpha^{3}(1-\alpha) \mathbb{E}\left(U_{t}^{2}\right) \mathbb{E}\left(U_{t-1}^{2}\right) \mathbb{E}\left(X_{t-2}\right)+\alpha(1-\alpha) \mathbb{E}\left(U_{t}^{2}\right) \mathbb{E}\left(X_{t-1}\right) \\
& +\alpha^{6} \mathbb{E}\left(U_{t}^{2}\right) \mathbb{E}\left(U_{t-1}^{2}\right) \operatorname{Var}\left(U_{t-2}^{2}\right) \mathbb{E}^{2}\left(X_{t-3}\right) \\
& +\alpha^{4} \mathbb{E}\left(U_{t}^{2}\right) \operatorname{Var}\left(U_{t-1}\right) \mathbb{E}^{2}\left(X_{t-2}\right)+\alpha^{2} \operatorname{Var}\left(U_{t}\right) \mathbb{E}^{2}\left(X_{t-1}\right)
\end{aligned}
$$


Desta forma, obtemos

$$
\begin{aligned}
\operatorname{Var}\left(X_{t}\right)= & \alpha^{2 k} \operatorname{Var}\left(X_{t-k}\right) \prod_{i=0}^{k-1} E\left(U_{t-i}^{2}\right)+\operatorname{Var}\left(\left(1-U_{t}\right) \varepsilon_{t}\right) \\
& +\sum_{j=1}^{k-1} \alpha^{2 j} \operatorname{Var}\left(\left(1-U_{t-j}\right) \varepsilon_{t-j}\right) \prod_{i=0}^{j-1} E\left(U_{t-i}^{2}\right) \\
& +(1-\alpha) \sum_{j=1}^{k} \alpha^{2 j-1} E\left(X_{t-j}\right) \prod_{i=0}^{j-1} E\left(U_{t-i}^{2}\right)+\alpha^{2} \operatorname{Var}\left(U_{t}\right) E^{2}\left(X_{t-1}\right) \\
& +\sum_{j=1}^{k-1} \alpha^{2(j+1)} E^{2}\left(X_{t-(j+1)}\right) \operatorname{Var}\left(U_{t-j}\right) \prod_{i=0}^{j-1} E\left(U_{t-i}^{2}\right) .
\end{aligned}
$$

\subsubsection{Processo DAR(1) com Binomial thinning correlacionado}

Assumamos que a seqüência $\left\{Y_{j}\right\}$ é composta por variáveis binárias equicorrelacionadas, no lugar de independentes. Usemos o operador binomial thinning correlacionado definido no capítulo anterior.

Este processo está definido em (4.11), sendo que o operador $\alpha * X_{t-1}$ foi substituído pelo operador binomial thinning correlacionado $\alpha \bullet_{\rho} X_{t-1}$, i.e.,

$$
\left\{\begin{array}{l}
X_{1}=\varepsilon_{1}, \\
X_{t}=U_{t}\left(\alpha \bullet_{\rho} X_{t-1}\right)+\left(1-U_{t}\right) \varepsilon_{t}, \quad 2 \leq t \leq m .
\end{array}\right.
$$

A estrutura de auto-covariância e a representação do processo estendido são dados de forma muito similar à sub-seção 4.2.1. Novamente, para garantir a estacionariedade de primeira ordem, consideremos inovações Poisson com parâmetro $\frac{\lambda(1-r \alpha)}{1-r}$, i.e., para o processo DAR(1) com binomial thinning correlacionado temos $E\left(X_{t}\right)=\lambda$. A variância é dada por 


$$
\begin{aligned}
\operatorname{Var}\left(X_{t}\right)= & \alpha^{2 k} \operatorname{Var}\left(X_{t-k}\right) \prod_{i=0}^{k-1} E\left(U_{t-i}^{2}\right)+\operatorname{Var}\left(\left(1-U_{t}\right) \varepsilon_{t}\right) \\
& +\sum_{j=1}^{k-1} \alpha^{2 j} \operatorname{Var}\left(\left(1-U_{t-j}\right) \varepsilon_{t-j}\right) \prod_{i=0}^{j-1} E\left(U_{t-i}^{2}\right) \\
& +(1-\alpha) \sum_{j=1}^{k} \alpha^{2 j-1} E\left(X_{t-j}\right) \prod_{i=0}^{j-1} E\left(U_{t-i}^{2}\right)+\alpha^{2} \operatorname{Var}\left(U_{t}\right) E^{2}\left(X_{t-1}\right) \\
& +\sum_{j=1}^{k-1} \alpha^{2(j+1)} E^{2}\left(X_{t-(j+1)}\right) \operatorname{Var}\left(U_{t-j}\right) \prod_{i=0}^{j-1} E\left(U_{t-i}^{2}\right) \\
& +\rho(1-\alpha) \sum_{j=1}^{k} \alpha^{2 j-1} E\left[X_{t-j}\left(X_{t-j}-1\right)\right] \prod_{i=0}^{j-1} E\left(U_{t-i}^{2}\right) .
\end{aligned}
$$

\subsubsection{Estimação dos parâmetros}

Os operadores aplicados aos processos (4.11) e (4.12) são independentes do passado histórico do processo. Em conseqüência, dizemos que os modelos DAR(1) estendidos têm propriedades Markovianas, i.e.,

$$
\mathbb{P}\left(X_{t} \mid x_{1}, \ldots, x_{t-1}\right)=\mathbb{P}\left(X_{t} \mid x_{t-1}\right)
$$

cuja distribuição conjunta pode ser expressa por

$$
\mathbb{P}\left(X_{t}, X_{t-1}, X_{t-2}, \ldots\right)=\mathbb{P}\left(X_{t} \mid x_{t-1}\right) \mathbb{P}\left(X_{t-1} \mid x_{t-2}\right) \ldots \mathbb{P}\left(X_{1}\right)
$$

Seja a função de verossimilhança da amostra $\mathrm{x}=\left(x_{1}, x_{2}, \ldots, x_{N}\right)$

$$
L_{N}(\mathrm{x}, \theta)=\mathbb{P}_{\theta}\left(X_{1}=x_{1}\right) \prod_{t=2}^{N} \mathbb{P}_{\theta}\left(x_{t-1}, x_{t}\right)
$$

em que $\theta \in \Theta$ é o vetor de parâmetros desconhecidos e $\mathbb{P}_{\theta}\left(x_{t-1}, x_{t}\right)$ são as correspondentes probabilidades de transição da cadeia de Markov. 
Desconsideremos a dependência do valor inicial e consideremos como um estimador para $\theta$ o estimador de máxima verossimilhança condicional dado $X_{1}=x_{1}$, que conseguimos maximizando a função de log-verossimilhança condicional sobre $\Theta$

$$
\log L_{N}(\mathrm{x}, \theta)=l_{N}\left(\mathrm{x}, \theta \mid x_{1}\right)=\sum_{t=2}^{N} \log \mathbb{P}_{\theta}\left(x_{t-1}, x_{t}\right)
$$

Apliquemos o método acima para os dois processos considerados com inovações Poisson e assumamos que $\varepsilon_{t} \sim P o\left(\frac{\lambda(1-r \alpha)}{1-r}\right)$. Então, o nosso vetor de parâmetros é dado por $\theta=(\alpha, r, \lambda, \rho)$.

Assim, as probabilidades de transição para o modelo (4.11) são calculadas da seguinte maneira

$$
\begin{aligned}
\mathbb{P}_{\theta}\left(x_{t-1}, x_{t}\right)= & \mathbb{P}\left(X_{t}=n \mid x_{t-1}=m\right) \\
= & \mathbb{P}\left(X_{t}=n \mid x_{t-1}=m, U_{t}=0\right) \mathbb{P}\left(U_{t}=0\right) \\
& +\mathbb{P}\left(X_{t}=n \mid x_{t-1}=m, U_{t}=1\right) \mathbb{P}\left(U_{t}=1\right) \\
= & \mathbb{P}\left[\left(1-U_{t}\right) \varepsilon_{t}+U_{t} \sum_{i=1}^{X_{t-1}} Y_{i}=n \mid X_{t-1}=m, U_{t}=0\right] \mathbb{P}\left(U_{t}=0\right) \\
& +\mathbb{P}\left[\left(1-U_{t}\right) \varepsilon_{t}+U_{t} \sum_{i=1}^{X_{t-1}} Y_{i}=n \mid X_{t-1}=m, U_{t}=1\right] \mathbb{P}\left(U_{t}=1\right) \\
= & \mathbb{P}\left(\varepsilon_{t}=n\right) \mathbb{P}\left(U_{t}=0\right)+\mathbb{P}\left(\sum_{i=1}^{m} Y_{i}=n\right) \mathbb{P}\left(U_{t}=1\right) .
\end{aligned}
$$

Portanto, $\mathbb{P}_{\theta}\left(x_{t-1}, x_{t}\right)$ fica dada por

$$
\mathbb{P}_{\theta}\left(x_{t-1}, x_{t}\right)=(1-r) \frac{e^{-\beta} \beta^{n}}{n !}+r\left(\begin{array}{c}
m \\
n
\end{array}\right) \alpha^{n}(1-\alpha)^{m-n} \mathbf{1}\{n \leq m\},
$$

sendo $\theta=(\alpha, r, \lambda), \beta=\frac{\lambda(1-r \alpha)}{1-r}$ e $1\{$.$\} a função indicadora.$

Analogamente, as probabilidades para o processo DAR(1) com o operador 
binomial thinning correlacionado (modelo (4.12)) são dadas por

$$
\begin{aligned}
\mathbb{P}_{\theta}\left(x_{t-1}, x_{t}\right)= & (1-r) \frac{e^{-\beta} \beta^{n}}{n !}+r\left\{(1-\rho)(1-\alpha)^{m}+\rho(1-\alpha)\right\} 1\{n=0\} \\
& +r(1-\rho)\left(\begin{array}{c}
m \\
n
\end{array}\right) \alpha^{n}(1-\alpha)^{m-n} \mathbf{1}\{1 \leq n \leq m-1\} \\
& +r\left\{(1-\rho) \alpha^{m}+\rho \alpha\right\} \mathbf{1}\{m=n\},
\end{aligned}
$$

sendo $\theta=(\alpha, r, \lambda, \rho)$ o vetor de parâmetros.

Denotemos o estimador de máxima verossimilhança condicional para o modelo (4.12) por $\hat{\theta}$, que foi obtido da solução das equações normais

$$
\frac{\partial l_{N}}{\partial \alpha}=0, \quad \frac{\partial l_{N}}{\partial r}=0 \quad \frac{\partial l_{N}}{\partial \lambda}=0 \quad \text { e } \quad \frac{\partial l_{N}}{\partial \rho}=0 .
$$

Desta maneira

$$
\begin{aligned}
\frac{\partial l_{N}}{\partial \alpha} & =\frac{\partial}{\partial \alpha} \sum_{t=1}^{N} \log \mathbb{P}_{\theta}\left(x_{t-1}, x_{t}\right)=\sum_{t=1}^{N} \frac{\partial}{\partial \alpha} \log \mathbb{P}_{\theta}\left(x_{t-1}, x_{t}\right) \\
& =\sum_{t=1}^{N}\left[\frac{1}{\mathbb{P}_{\theta}} \frac{\partial \mathbb{P}_{\theta}}{\partial \alpha}\right],
\end{aligned}
$$

sendo

$$
\begin{aligned}
\frac{\partial \mathbb{P}_{\theta}}{\partial \alpha}= & \lambda r e^{-\beta} \sum_{t=2}^{N}\left[-\frac{\beta^{x_{t}}}{x_{t} !}+\frac{\beta^{x_{t}-1}}{\left(x_{t}-1\right) !}\right] \\
& +r e^{-\beta} \sum_{t=2}^{N}\left(\begin{array}{c}
x_{t-1} \\
x_{t}
\end{array}\right) \alpha^{x_{t-1}}(1-\alpha)^{x_{t-1}-x_{t}-1}\left(x_{t}-\alpha x_{t-1}\right) 1\left\{x_{t} \leq x_{t-1}\right\} .
\end{aligned}
$$

As outras derivadas são calcualadas de forma análoga (veja Apêndice C).

Observemos que $\hat{\theta}=(\hat{\alpha}, \hat{r}, \hat{\lambda}, \hat{\rho})$ não é necessariamente a única solução do último sistema de equações. Devido à complexidade das equações, não foi possível achar a forma explícita das expressões para o estimador $\hat{\theta}$. Portanto, usamos o método de Newton-Raphson para estimar os parâmetros do modelo. 
Os valores iniciais foram calculados de forma muito similar ao método usado no processo CINAR(1), i.e., foram obtidos minimizando $\sum_{t=2}^{N}\left[X_{t}-\mathbb{E}\left(X_{t} \mid x_{t-1}\right)\right]^{2}$. Mantivemos estes valores tanto para o modelo (4.11) como para o modelo (4.12).

\subsubsection{Ilustração}

A série de dados corresponde ao número diário de ataques epilépticos de um paciente durante 204 dias [Mac Donald e Zucchini (1997), página 208].

A seguir, apresentamos os gráficos das séries originais, das séries dos resíduos e das funções de autocorrelação dos resíduos para os dois modelos. 
Grafico da Serie

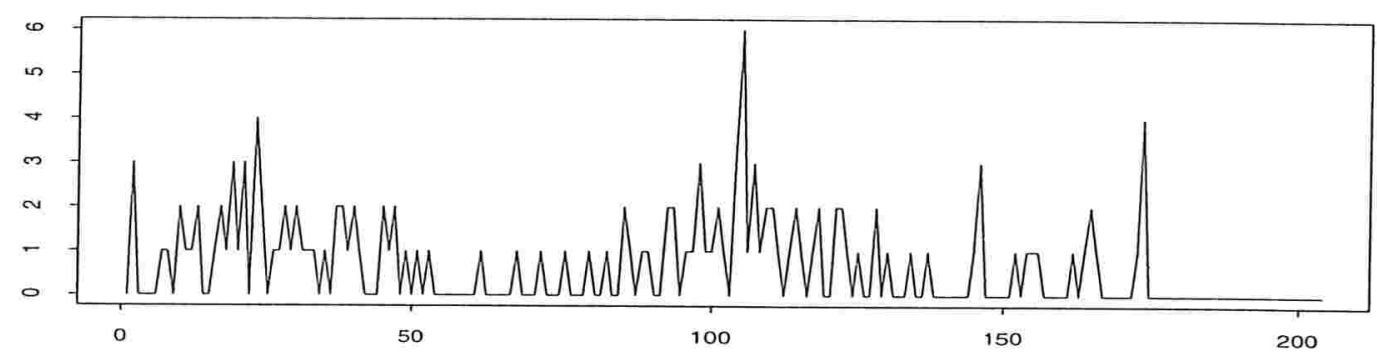

Grafico dos Residuos

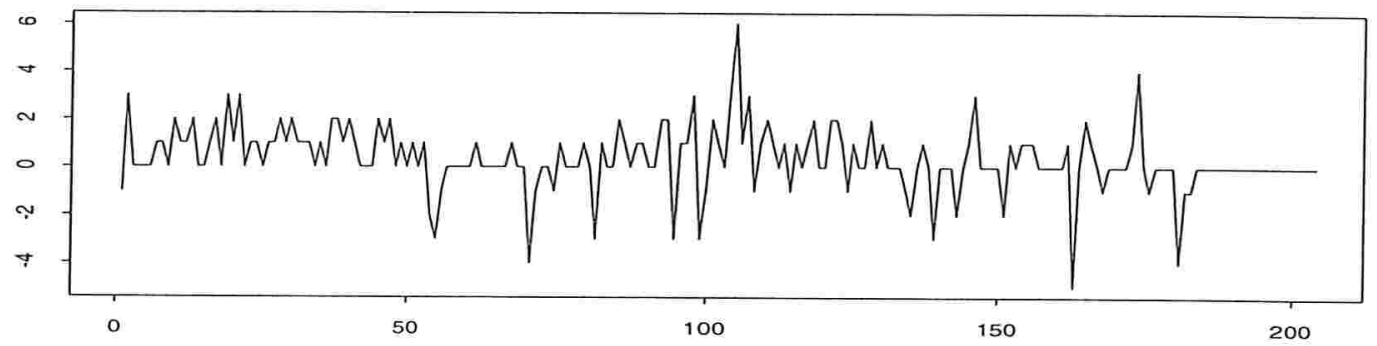

Funcao de Autocorrelacao dos Residuos

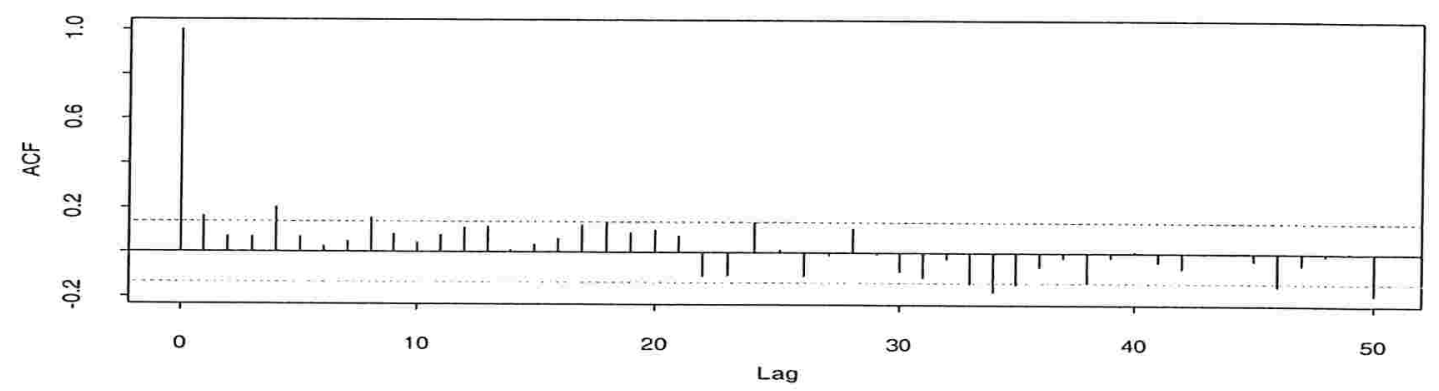

Figura 4.5: Gráficos da série original, dos resíduos e da função de autocorrelação dos resíduos, para o modelo $D A R(1)$ com Binomial thinning. 
Grafico da Serie

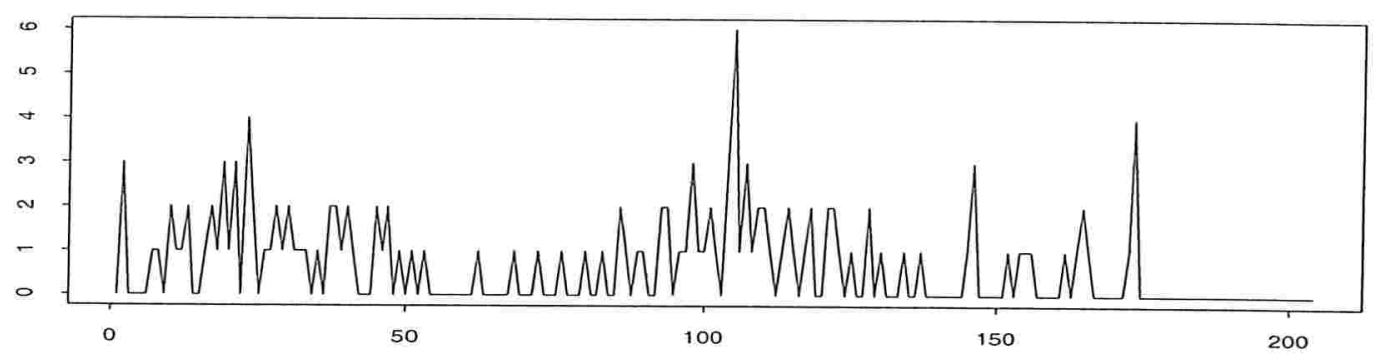

Grafico dos Residuos

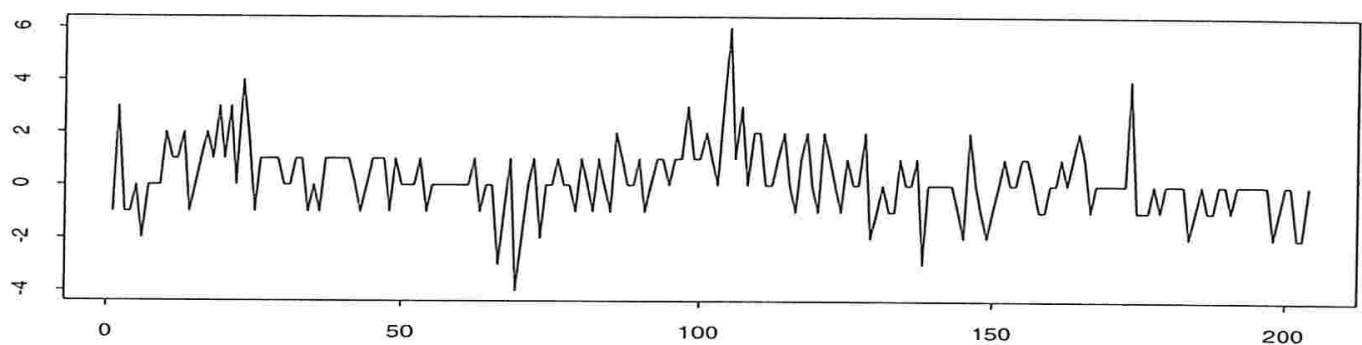

Funcao de Autocorrelacao dos Residuos

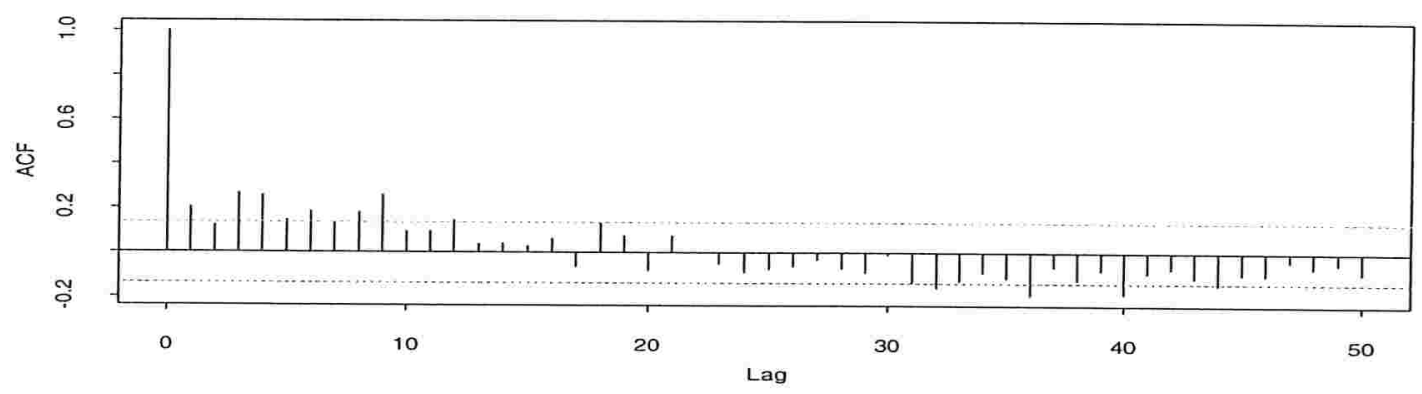

Figura 4.6: Gráficos da série original, dos resíduos e da função de autocorrelação dos resíduos, para o modelo DAR(1) com Binomial thinning correlacionado.

Nas Figuras 4.5 e 4.6, apresentamos os gráficos da série original, dos resíduos e as funções de autocorrelação dos dois modelos DAR(1) estendidos. Observemos que a série original apresenta diferentes variabilidades, o mesmo ocorrendo para as 
séries dos resíduos dos dois modelos. As funções de autocorrelação dos resíduos se aproximam das funções de autocorrelação do ruído branco.

As estimativas e os valores dos desvios padrão são dados na Tabela 4.3, onde comparamos os modelos (4.11) e (4.12) para o número de pacientes com epilepsia.

Tabela 4.3: Valores iniciais e estimativas dos parâmetros dos modelos (4.11) e (4.12).

\begin{tabular}{cccccccccccc}
\hline \multicolumn{4}{c}{ Valores iniciais } & \multicolumn{4}{c}{$\operatorname{Modelo}(4.11)$} & \multicolumn{4}{c}{$\operatorname{Modelo}(4.12)$} \\
\hline$\alpha_{0}$ & $\lambda_{0}$ & $r_{0}$ & $\rho_{0}$ & $\hat{\alpha}$ & $\hat{\lambda}$ & $\hat{r}$ & $\hat{\alpha}$ & $\hat{\lambda}$ & $\hat{r}$ & $\hat{\rho}$ \\
\hline 0.29 & 0.66 & 0.81 & 0.01 & 0.26 & 0.32 & 0.84 & 0.42 & 0.65 & 0.82 & 0.78 \\
& & & & $(0,17)$ & $(0,09)$ & $(0,07)$ & $(0,11)$ & $(0,02)$ & $(0,01)$ & $(0,19)$ \\
\hline
\end{tabular}

Observemos que o valor estimado 0,78 do parâmetro adicional $\rho$ indica a presença de dependência dentro dos $Y_{i}$ 's. O programa utilizado encontra-se no Apêndice C.

Para a escolha do modelo, usamos os critérios AIC e BIC (veja, Shumway (2000)). Obtivemos os seguintes resultados

Tabela 4.4: Valores dos coeficientes AIC e BIC para os Modelos (4.11) e (4.12).

\begin{tabular}{lccccc}
\hline & $\sigma_{a}^{2}$ & $\begin{array}{c}\text { Número de } \\
\text { parâmetros }\end{array}$ & $\begin{array}{c}\text { Tamanho da } \\
\text { amostra }\end{array}$ & AIC & BIC \\
\hline Modelo (4.11) & 1,72 & 3 & 204 & 0,58 & 0,63 \\
\hline Modelo (4.12) & 1,62 & 4 & 204 & 0,52 & 0,59 \\
\hline
\end{tabular}

Verificamos, tanto pela variância residual $\left(\sigma_{a}^{2}\right)$ como pelos valores dos critérios de AIC e BIC, que o modelo correlacionado (4.12) é melhor do que o modelo 
(4.11), o que indica a presença de dependência entre os $Y_{j}$ 's, pois o valor estimado de correlação foi $\hat{\rho}=0,78$.

Neste capítulo, estendemos os processos INAR(1) e DAR(1) para o caso de v.a.'s igualmente correlacionadas. Podemos concluir que tanto o processo CINAR(1) como os processos DAR(1) estendidos apresentam estimativas de correlação bastante significativas. Isto significa que os modelos que consideram v.a.'s dependentes apresentam um melhor ajuste do que os modelos com v.a.'s independentes. 


\section{Capítulo 5}

\section{Modelo multinomial latente para somas aleatórias}

Neste capítulo, apresentaremos um modelo novo que generaliza resultados clássicos já existentes. Consideraremos sinistros distribuídos em clusters independentes e assumiremos que existe dependência só entre sinistros que pertencem ao mesmo cluster. Mediremos esta dependência como até agora, com o coeficiente de correlação. Para modelar esta situação, utilizaremos a distribuição multinomial.

Na seção 5.1, apresentaremos o modelo geral (multinomial correlacionado), que é uma extensão natural mais próxoma da realidade. Um caso particular na presença de dois clusters será detalhado na seção 5.2. Neste caso, a distribuição proposta poderá ser expressa como uma mistura de variáveis aleatórias binomiais, o que facilitará os cálculos dos estimadores de máxima veossimilhança.

Na seção 5.3, obteremos as estimativas dos parâmetros envolvidos na seção 5.2 por meio do método de máxima verossimilhança. Além disso, apresentaremos algumas simulações. 


\subsection{Modelo multinomial correlacionado}

No capítulo 2, vimos que o modelo binomial correlacionado assume implicitamente que as observações dependentes (equicorrelacionadas) pertencem a um cluster só. Na maioria dos casos práticos, os dados são agrupados em clusters (classes) independentes, com uma possível estrutura de dependência entre as observações de um mesmo cluster.

Para modelar este caso de forma mais realística, suponhemos que os nossos $n$ dados são agrupados em $K$ clusters independentes, $2 \leq K \leq n$, de acordo com o seguinte mecanismo: cada observação é atribuída com probabilidade $p_{k}$ ao $k$-ésimo cluster, $(k=1,2, \ldots, K)$ e $\sum_{k=1}^{K} p_{k}=1$. No fim da atribuição, o $k$-ésimo clustèr conterá $n_{k}$ elementos. Neste caso, o vetor $\mathbf{n}=\left(n_{1}, n_{2}, \ldots, n_{K}\right)$ tem distribuição Multinomial com parâmetros

$$
n=n_{1}+n_{2}+\ldots+n_{K} \quad \text { e } \quad \mathrm{p}=\left(p_{1}, p_{2}, \ldots, p_{K}\right),
$$

i.e., $\mathrm{n} \sim M n(n, \mathbf{p})$, que pode ser considerado como uma viariável latente. Suponhemos que os sinistros $Y_{k j}$ pertencentes ao $k$-ésimo cluster $(k=1,2, \ldots, K$; $\left.j=1,2, \ldots, n_{k}\right)$ são equicorrelacionados, com correlação dada por

$$
\rho_{k}=\operatorname{Corr}\left(Y_{k j}, Y_{k j^{\prime}}\right) \in\left[\max \left\{-\frac{p_{k}}{1-p_{k}},-\frac{1-p_{k}}{p_{k}},-\frac{1}{1-n_{k}}\right\}, 1\right],
$$

para $j \neq j^{\prime}$ e $k=1,2, \ldots, K$. As v.a.'s indicadoras $Z_{k j}=1\left\{Y_{k j}>M\right\}$ têm a mesma distribuição Bernoulli com parâmetros

$$
\alpha_{k}=\mathbb{P}\left(Y_{k j}>M\right), \quad k=1,2, \ldots, K ; \quad j=1,2, \ldots, n_{k},
$$

e são equicorrelacionados $\operatorname{com} \rho_{k}=\operatorname{Corr}\left(Z_{k j}, Z_{k j^{\prime}}\right)$, de acordo com as condições do Lema 2.1. Então, o número total de observações

$$
S_{n_{k}}=\sum_{j=1}^{n_{k}^{\prime}} Z_{k_{j},}, k=1,2, \ldots, \underline{K} ;
$$


assinado dentro do $k$-ésimo cluster, para o qual a resseguradora deve pagar num contrato de resseguro "stop-loss" com retenção $M>0$, tem distribuição binomial correlacionada com parâmetros $n_{k}, \alpha_{k}$ e $\rho_{k}$, i.e., $S_{n_{k}} \sim \operatorname{CBi}\left(n_{k}, \alpha_{k}, \rho_{k}\right)$, cuja FGP, $G_{S_{n_{k}}}(t)$, pode ser representada por $(2.22)$.

Portanto, assumindo que os $K$ clusters são independentes, obtemos o seguinte resultado:

\section{Teorema 5.1.}

A FGP da soma acumulada $\sum_{k=1}^{K} S_{n_{k}}$ pode ser representada por

$$
G_{S_{n}}(t)=\mathbb{E}_{\mathbf{n}}\left[\prod_{k=1}^{K} G_{S_{n_{k}}}(t)\right],
$$

sendo que a esperança $\mathbb{E}_{\mathbf{n}}(\cdot)$ é relativa ao vetor aleatório multinomial $\mathbf{n} \sim M n(n, \mathbf{p})$ e $G_{S_{n_{k}}}(t)$ é a FGP dada em (2.22).

Usando a fórmula de probabilidade total e (5.1), podemos obter a distribuição da soma acumulada para a resseguradora, supondo a distribuição $N$ conhecida.

\subsubsection{Um caso particular de dois clusters}

Quando o número de clusters é grande, torna-se difícil obter de (5.1) uma forma explícita para a distribuição de $S_{n}$. Nesta seção, consideraremos o caso particular de observações agrupadas em dois clusters. Neste caso, o vetor $\mathbf{n}=\left(n_{1}, n_{2}\right)$ tem distribuição Binomial com parâmetros $n$ e $p_{1}$, i.e., $\mathbf{n} \sim B i\left(n, p_{1}\right)$, com $n=n_{1}+n_{2}$.

Estamos interessados em obter a distribuição exata de $S_{n}=S_{n_{1}}+S_{n_{2}}$ quando $S_{n_{k}} \sim \operatorname{CBi}\left(n_{k}, \alpha_{k}, \rho_{k}\right)$, para $k=1,2, \operatorname{com} \rho_{1} \neq \rho_{2}$ e $\alpha_{1} \neq \alpha_{2}$.

De (5.1), temos que a FGP de $S_{n}$ é dada por

$$
G_{S_{n}}(t)=\mathbb{E}_{\mathbf{n}}\left[G_{S_{n_{1}}}(t) G_{S_{n_{2}}}(t)\right] .
$$


Logo, usando (5.2) e a fórmula de probabilidade total para uma v.a. binomial $V \sim B i\left(n, p_{1}\right)$, temos

$$
\begin{aligned}
G_{S_{n}}(t)= & \sum_{n_{1}=1}^{n}\left[G_{S_{n_{1}}}(t) G_{S_{n_{2}}}(t) \mid V=n_{1}\right] P\left(V=n_{1}\right) \\
= & \sum_{n_{1}=0}^{n}\left\{\prod_{k=1}^{2}\left[\rho_{k}\left(1-\alpha_{k}+\alpha_{k} t^{n}\right)+\left(1-\rho_{k}\right)\left(1-\alpha_{k}+\alpha_{k} t\right)^{n_{k}}\right]\right\} \times \\
& \times\left(\begin{array}{c}
n \\
n_{1}
\end{array}\right) p_{1}^{n_{1}}\left(1-p_{1}^{n-n_{1}}\right) .
\end{aligned}
$$

Após algumas manipulações algébricas, obtemos:

$$
\begin{aligned}
G_{S_{n}}(t)= & \rho_{1} \rho_{2}\left(1-\alpha_{1}\right)\left(1-\alpha_{2}\right)+\rho_{1} \rho_{2} \alpha_{1} \alpha_{2} t^{n} \\
& +\rho_{1} \rho_{2}\left(1-\alpha_{1}\right) \alpha_{2}\left[p_{1}+\left(1-p_{1}\right) t\right]^{n}+\rho_{1} \rho_{2} \alpha_{1}\left(1-\alpha_{2}\right)\left[1-p_{1}+p_{1} t\right]^{n} \\
& +\left(1-\rho_{1}\right)\left(1-\rho_{2}\right)\left[1-\alpha_{2}+\alpha_{2} p_{1}-\alpha_{1} p_{1}+\left(\alpha_{2}-\alpha_{2} p_{1}+\alpha_{1} p_{1}\right) t\right]^{n} \\
& +\left(1-\rho_{1}\right) \rho_{2}\left(1-\alpha_{2}\right)\left[1-\alpha_{1} p+\alpha_{1} p_{1} t\right]^{n} \\
& +\rho_{1}\left(1-\rho_{2}\right)\left(1-\alpha_{1}\right)\left[1-\alpha_{2}+\alpha_{2} p_{1}+\left(\alpha_{2}-\alpha_{2} p_{1}\right) t\right]^{n} \\
& +\left(1-\rho_{1}\right) \rho_{2} \alpha_{2} p_{1}\left[\alpha_{1}-\alpha_{1} p_{1}+\left(1-\alpha_{1}+\alpha_{1} p_{1}\right) t\right]^{n} \\
& +\rho_{1}\left(1-\rho_{2}\right) \alpha_{1}\left[1-\alpha_{2}-p_{1}+\alpha_{2} p_{1}+\left(\alpha_{2}+p_{1}-\alpha_{2} p_{1}\right) t\right]^{n} .
\end{aligned}
$$

Observemos que esta última expressão pode ser escrita como uma mistura da forma

$$
G_{S_{n}}(t)=\sum_{l=1}^{9} a_{l} G_{U_{l}}(t)
$$

em que $\sum_{l=1}^{9} a_{l}=1, U_{1}=0$ e $U_{2}=n$ e as v.a.'s $U_{l}(l=3,4, \ldots, 9)$ são binomiais de parâmetros $n$ e $\beta_{l}$, respectivamente.

Notemos que, se consideramos $K$ clusters, a FGP de $S_{n}$ conterá $(K+1)^{K}$ termos. 
Os valores dos coeficientes $a_{l}$ e os parâmetros $\beta_{l}$ das v.a.'s $U_{l}$ são dados na Tabela 5.1.

Tabela 5.1: Coeficientes da mistura e parâmetros das variáveis aleatórias $U_{l}$ para o modelo (5.3).

\begin{tabular}{lll}
\hline Variável & $a_{l}$ & $\beta_{l}$ \\
\hline$U_{1}$ & $\rho_{1} \rho_{2}\left(1-\alpha_{1}\right)\left(1-\alpha_{2}\right)$ & 0 \\
$U_{2}$ & $\rho_{1} \rho_{2} \alpha_{1} \alpha_{2}$ & 1 \\
$U_{3}$ & $\rho_{1} \rho_{2}\left(1-\alpha_{1}\right) \alpha_{2}$ & $1-p_{1}$ \\
$U_{4}$ & $\rho_{1} \rho_{2} \alpha_{1}\left(1-\alpha_{2}\right)$ & $p_{1}$ \\
$U_{5}$ & $\left(1-\rho_{1}\right)\left(1-\rho_{2}\right)$ & $\alpha_{2}-\alpha_{2} p_{1}+\alpha_{1} p_{1}$ \\
$U_{6}$ & $\left(1-\rho_{1}\right) \rho_{2}\left(1-\alpha_{2}\right)$ & $\alpha_{1} p_{1}$ \\
$U_{7}$ & $\rho_{1}\left(1-\rho_{2}\right)\left(1-\alpha_{1}\right)$ & $\alpha_{2}-\alpha_{2} p_{1}$ \\
$U_{8}$ & $\left(1-\rho_{1}\right) \rho_{2} \alpha_{2}$ & $1-\alpha_{1}+\alpha_{1} p_{1}$ \\
$U_{9}$ & $\rho_{1}\left(1-\rho_{2}\right) \alpha_{1}$ & $\alpha_{2}+p_{1}-\alpha_{2} p_{1}$ \\
\hline
\end{tabular}

Chamemos o modelo (5.3) de Modelo $S$, cujo vetor de parâmetros é dado por $\Theta=\left(n, p_{1}, \alpha_{1}, \alpha_{2}, \rho_{1}, \rho_{2}\right)$.

Agora, restrinjamos nosso modelo (5.3) a três casos particulares.

Caso 1: Seja $\alpha_{1}=\alpha_{2}=\alpha$ e $\rho_{1}=\rho_{2}=\rho$, i.e., o vetor de parâmetros do modelo é dado por $\Theta=\left(n, p_{1}, \alpha, \rho\right)$. Chamemos este modelo de Modelo S1.

Caso 2: Assumamos que $\rho_{1}=\rho_{2}=\rho$ e $\alpha_{1} \neq \alpha_{2}$. Assim, o vetor de parâmetros do modelo é dado por $\Theta=\left(n, p_{1}, \alpha_{1}, \alpha_{2}, \rho\right)$. Denominemos este modelo por Modelo S2.

Caso 3: Consideremos $\rho_{1} \neq \rho_{2}$ e $\alpha_{1}=\alpha_{2}=\alpha$, i.e., o vetor de parâmetros é dado 
por $\Theta=\left(n, p_{1}, \alpha, \rho_{1}, \rho_{2}\right)$. Denotemos este modelo por $\mathrm{S} 3$.

Da maneira análoga ao Modelo S, é possível achar as FGP's de cada um dos casos particulares Desta forma, veremos que cada modelo pode ser dado como uma mistura.

A seguir, apresentamos duas tabelas que nos mostrarão de forma resumida os coeficientes e parâmetros dos três casos particulares. A Tabela 5.2 nos fornece os coeficientes de mistura $a_{l}$, para os modelos $S 1, S 2$ e $S 3$, e a Tabela 5.3 nos mostra os valores dos parâmetros $\beta_{l}$ das v.a's $U_{l} \sim B i\left(n, \beta_{l}\right), l=3,4, \ldots, 9$ (lembremos que $U_{1}=0$ e $U_{2}=n$ ), também para os modelos $S 1$, $S 2$ e $S 3$.

Tabela 5.2: Coeficientes de mistura dos modelos.

\begin{tabular}{llll}
\hline Variável & Modelo $S 1$ & Modelo $S 2$ & Modelo $S 3$ \\
\hline$U_{1}$ & $\rho^{2}(1-\alpha)^{2}$ & $\rho^{2}\left(1-\alpha_{1}\right)\left(1-\alpha_{2}\right)$ & $\rho_{1} \rho_{2}(1-\alpha)^{2}$ \\
$U_{2}$ & $\rho^{2} \alpha^{2}$ & $\rho^{2} \alpha_{1} \alpha_{2}$ & $\rho_{1} \rho_{2} \alpha^{2}$ \\
$U_{3}$ & $\rho^{2} \alpha(1-\alpha)$ & $\rho^{2}\left(1-\alpha_{1}\right) \alpha_{2}$ & $\rho_{1} \rho_{2} \alpha(1-\alpha)$ \\
$U_{4}$ & $\rho^{2} \alpha(1-\alpha)$ & $\rho^{2} \alpha_{1}\left(1-\alpha_{2}\right)$ & $\rho_{1} \rho_{2} \alpha(1-\alpha)$ \\
$U_{5}$ & $(1-\rho)^{2}$ & $(1-\rho)^{2}$ & $\left(1-\rho_{1}\right)\left(1-\rho_{2}\right)$ \\
$U_{6}$ & $\rho(1-\rho)(1-\alpha)$ & $\rho(1-\rho)\left(1-\alpha_{2}\right)$ & $\rho_{1}\left(1-\rho_{2}\right)(1-\alpha)$ \\
$U_{7}$ & $\rho(1-\rho)(1-\alpha)$ & $\rho(1-\rho)\left(1-\alpha_{1}\right)$ & $\rho_{1}\left(1-\rho_{2}\right)(1-\alpha)$ \\
$U_{8}$ & $\rho(1-\rho) \alpha$ & $\rho(1-\rho) \alpha_{2}$ & $\rho_{1}\left(1-\rho_{2}\right) \alpha$ \\
$U_{9}$ & $\rho(1-\rho) \alpha$ & $\rho(1-\rho) \alpha_{1}$ & $\rho_{1}\left(1-\rho_{2}\right) \alpha$ \\
\hline
\end{tabular}


Tabela 5.3: Parâmetros $\beta_{l}$ das variáveis aleatórias $U_{l}, l=3,4, \ldots, 9$.

\begin{tabular}{llll}
\hline Variável & Modelo $S 1$ & Modelo $S 2$ & Modelo $S 3$ \\
\hline$U_{3}$ & $1-p_{1}$ & $1-p_{1}$ & $1-p_{1}$ \\
$U_{4}$ & $p_{1}$ & $p_{1}$ & $p_{1}$ \\
$U_{5}$ & $\alpha$ & $\alpha_{2}-\alpha_{2} p_{1}+\alpha_{1} p_{1}$ & $\alpha$ \\
$U_{6}$ & $\alpha p_{1}$ & $\alpha_{1} p_{1}$ & $\alpha p_{1}$ \\
$U_{7}$ & $\alpha-\alpha p_{1}$ & $\alpha_{2}-\alpha_{2} p_{1}$ & $\alpha-\alpha p_{1}$ \\
$U_{8}$ & $1-\alpha+\alpha p_{1}$ & $1-\alpha_{1}+\alpha_{1} p_{1}$ & $1-\alpha-\alpha p_{1}$ \\
$U_{9}$ & $\alpha+p_{1}-\alpha p_{1}$ & $\alpha_{2}+p_{1}-\alpha_{2} p_{1}$ & $\alpha+p_{1}-\alpha p_{1}$ \\
\hline
\end{tabular}

O nosso objetivo agora é calcular as estimativas dos parâmetros para cada um dos modelos.

\subsection{Estimação dos parâmetros e simulação}

Muitos métodos para a estimação dos parâmetros de distribuições de mistura têm sido usados na literatura. Por exemplo, Blishke (1962) usou o método de momentos fatoriais para mistura de binomiais. No nosso caso, não foi possível aplicar diretamente este método, pois a mistura (5.3) contém duas v.a.'s indicadoras. Nós usamos o método de máxima verossimilhaça para estimar os parâmetros dos modelos $S$, $S 1$, $S 2$ e $S 3$. Para o modelo $S$, de (5.3) obtemos a seguinte distribuição da soma $S_{n}$ : 


$$
\begin{aligned}
\mathbb{P}\left(S_{n}=n_{1}\right)= & \rho_{1} \rho_{2}\left(1-\alpha_{1}\right)\left(1-\alpha_{2}\right) 1\left\{n_{1}=0\right\}+\rho_{1} \rho_{2} \alpha_{1} \alpha_{2} 1\left\{n_{1}=n\right\} \\
& +\rho_{1} \rho_{2}\left(1-\alpha_{1}\right) \alpha_{2}\left(\begin{array}{c}
n \\
n_{1}
\end{array}\right)\left(1-p_{1}\right)^{n_{1}} p_{1}^{n-n_{1}} \\
& +\rho_{1} \rho_{2} \alpha_{1}\left(1-\alpha_{2}\right)\left(\begin{array}{c}
n \\
n_{1}
\end{array}\right) p_{1}^{n_{1}}\left(1-p_{1}\right)^{n-n_{1}} \\
& +\left(1-\rho_{1}\right)\left(1-\rho_{2}\right)\left(\begin{array}{c}
n \\
n_{1}
\end{array}\right)\left(\alpha_{2}-\alpha_{2} p_{1}+\alpha_{1} p_{1}\right)^{n_{1}}\left(1-\alpha_{2}+\alpha_{2} p_{1}-\alpha_{1} p_{1}\right)^{n-n_{1}} \\
& +\left(1-\rho_{1}\right) \rho_{2}\left(1-\alpha_{2}\right)\left(\begin{array}{c}
n \\
n_{1}
\end{array}\right)\left(\alpha_{1} p_{1}\right)^{n_{1}}\left(1-\alpha_{1} p_{1}\right)^{n-n_{1}} \\
& +\rho_{1}\left(1-\rho_{2}\right)\left(1-\alpha_{1}\right)\left(\begin{array}{c}
n \\
n_{1}
\end{array}\right)\left(\alpha_{2}-\alpha_{2} p_{1}\right)^{n_{1}}\left(1-\alpha_{2}+\alpha_{2} p_{1}\right)^{n-n_{1}} \\
& +\left(1-\rho_{1}\right) \rho_{2} \alpha_{2}\left(\begin{array}{c}
n \\
n_{1}
\end{array}\right)\left(1-\alpha_{1}+\alpha_{1} p_{1}\right)^{n_{1}}\left(\alpha_{1}-\alpha_{1} p_{1}\right)^{n-n_{1}} \\
& +\rho_{1}\left(1-\rho_{2}\right) \alpha_{1}\left(\begin{array}{c}
n \\
n_{1}
\end{array}\right)\left(\alpha_{2}+p_{1}-\alpha_{2} p_{1}\right)^{n_{1}}\left(1-\alpha_{2}-p_{1}+\alpha_{2} p_{1}\right)^{n-n_{1}}
\end{aligned}
$$

em que $1\{\cdot\}$ representa a função indicadora e $n_{1}=0,1, \ldots, n$.

Observemos que o vetor de parâmetros deste modelo é denotado por $\Theta=\left(p_{1}, \alpha_{1}, \alpha_{2}, \rho_{1}, \rho_{2}\right)$. Os estimadores de máxima verossimilhança de $\Theta$ são obtidos maximizando a função (5.4), que é a solução do sistema de equações $\frac{\partial f}{\partial p_{1}}=0, \frac{\partial f}{\partial \alpha_{1}}=0, \frac{\partial f}{\partial \alpha_{2}}=0, \frac{\partial f}{\partial \rho_{1}}=0$ e $\frac{\partial f}{\partial \rho_{2}}=0$ sendo que, $f=\mathbb{P}\left(S_{n}=n_{1}\right)$. Os cálculos das derivadas são dados no Apêndice D.

Devido à complexidade das derivadas $\frac{\partial f}{\partial p_{1}}, \frac{\partial f}{\partial \alpha_{1}}, \frac{\partial f}{\partial \alpha_{2}}, \frac{\partial f}{\partial \rho_{1}}$ e $\frac{\partial f}{\partial \rho_{2}}$, não foi possível obter uma solução explícita para as equações de log-verossimilhança. Portanto, foi necessário usar um método iterativo. Adotamos o método iterativo de NewtonRaphson, para o qual foi preciso fornecer as primeiras e segundas derivadas da função. As estimativas foram obtidas mediante simulação.

Para obter as estimativas dos parâmetros dos modelos $S, S 1, S 2$ e $S 3$ propostos na seção 5.2, foram efetuadas simulações usando a linguagem de programação OX, 
veja Doornik (1996).

Geramos dois conjuntos de observações agrupados em dois clusters independentes de tamanhos $n_{1}(80$ e 30$)$ e $n_{2}(90$ e 140$), n=n_{1}+n_{2}=170$. Para o primeiro conjunto, consideramos $n_{1}$ e $n_{2}$ parecidos e para o segundo conjunto assumimos $n_{1}$ e $n_{2}$ diferentes. Levamos em consideração a dependência (equicorrelação) dos dados em cada cluster relativos aos modelos $S, S 1, S 2$ e $S 3$.

Para cada simulação, usamos os mesmos valores iniciais dos parâmetros $\alpha_{1}, \alpha_{2}, \rho_{1}$, $\rho_{2}$, fixando $p_{1}=0,5$. Os programas da simulação são dados no Apêndice D.

As Tabelas 5.4, 5.5, 5.6 e 5.7 mostram os resultados das simulações feitas para obter as estimativas e desvios padrão dos modelos $S, S 1, S 2$ e $S 3$.

Tabela 5.4: Valores iniciais e valores das estimativas do Modelo $S$.

\begin{tabular}{ccccccccccccc}
\hline & \multicolumn{1}{c}{ Valores iniciais } & \multicolumn{4}{c}{ Estimativas dos parâmetros } \\
\hline$n_{1}$ & $n_{2}$ & $p_{1}$ & $\alpha_{1}$ & $\alpha_{2}$ & $\rho_{1}$ & $\rho_{2}$ & $\hat{p_{1}}$ & $\hat{\alpha_{1}}$ & $\hat{\alpha_{2}}$ & $\hat{\rho_{1}}$ & $\hat{\rho_{2}}$ \\
\hline 80 & 90 & 0,50 & 0,52 & 0,39 & 0,62 & 0,29 & 0,38 & 0,47 & 0,47 & 0,73 & 0,73 \\
& & & & & & & $(0,04)$ & $(0,12)$ & $(0,11)$ & $(0,08)$ & $0,06)$ \\
30 & \multirow{2}{*}{140} & 0,50 & 0,52 & 0,39 & 0,62 & 0,29 & 0,81 & 0,83 & 0,07 & 0,92 & 0,30 \\
& & & & & & & $(0,08)$ & $(0,03)$ & $(0,19)$ & $(0,01)$ & $0,13)$ \\
\hline
\end{tabular}

Tabela 5.5: Valores iniciais e valores das estimativas do Modelo $S 1$.

\begin{tabular}{cccccccc}
\hline & & \multicolumn{3}{c}{ Valores iniciais } & \multicolumn{3}{c}{ Estimativas dos parâmetros } \\
\hline$n_{1}$ & $n_{2}$ & $p_{1}$ & $\alpha$ & $\rho$ & $\hat{p}_{1}$ & $\hat{\alpha}$ & $\hat{\rho}$ \\
\hline 80 & 90 & 0,50 & 0,80 & 0,70 & 0,43 & 0,82 & 0,60 \\
& & & & & $(0,16)$ & $(0,25)$ & $(0,01)$ \\
30 & 140 & 0,50 & 0,80 & 0,70 & 0,50 & 0,59 & 0,53 \\
& & & & & $(0,07)$ & $(0,12)$ & $(0,08)$ \\
\hline
\end{tabular}


Tabela 5.6: Valores iniciais e valores das estimativas do Modelo $S 2$.

\begin{tabular}{cccccccccc}
\hline & \multicolumn{4}{c}{ Valores iniciais } & \multicolumn{4}{c}{ Estimativas dos parâmetros } \\
\hline$n_{1}$ & $n_{2}$ & $p_{1}$ & $\alpha_{1}$ & $\alpha_{2}$ & $\rho$ & $\hat{p}_{1}$ & $\hat{\alpha_{1}}$ & $\hat{\alpha_{2}}$ & $\hat{\rho}$ \\
\hline 80 & 90 & 0,50 & 0,58 & 0,32 & 0,41 & 0,68 & 0,63 & 0,32 & 0,71 \\
& & & & & & $(0,03)$ & $(0,16)$ & $(0,21)$ & $(0,09)$ \\
30 & 140 & 0,50 & 0,58 & 0,32 & 0,41 & 0,58 & 0,09 & 0,83 & 0,99 \\
& & & & & & $(0,07)$ & $(0,24)$ & $(0,16)$ & $(0,01)$ \\
\hline
\end{tabular}

Tabela 5.7: Valores iniciais e valores das estimativas do Modelo S3.

\begin{tabular}{|c|c|c|c|c|c|c|c|c|c|}
\hline \multirow[b]{2}{*}{$n_{1}$} & \multirow[b]{2}{*}{$n_{2}$} & \multicolumn{4}{|c|}{ Valores iniciais } & \multicolumn{4}{|c|}{ Estimativas dos parâmetros } \\
\hline & & $p_{1}$ & $\alpha$ & $\rho_{1}$ & $\rho_{2}$ & $\hat{p_{1}}$ & $\hat{\alpha}$ & $\hat{\rho_{1}}$ & $\hat{\rho_{2}}$ \\
\hline \multirow[t]{2}{*}{80} & 90 & 0,50 & 0,65 & 0,45 & 0,71 & 0,62 & 0,58 & 0,30 & 0,74 \\
\hline & & & & & & $(0,05)$ & $(0,17)$ & $(0,02)$ & $(0,14)$ \\
\hline \multirow[t]{2}{*}{30} & 140 & 0,50 & 0,65 & 0,45 & 0,71 & 0,49 & 0,91 & 0,39 & 0,92 \\
\hline & & & & & & $(0,06)$ & $(0,08)$ & $(0,11)$ & $(0,03)$ \\
\hline
\end{tabular}

Observemos que quando os valores de $n_{1}$ e $n_{2}$ são próximos (80 e 90), as estimativas dos parâmetros $\hat{\rho_{1}}, \hat{\rho_{2}}$ e $\hat{\alpha_{1}}, \hat{\alpha_{2}}$ são iguais para o modelo $S$. Quando os tamanhos dos clusters são diferentes $\left(n_{1}=30\right.$ e $\left.n_{2}=140\right)$, as estimativas dos parâmetros também são diferentes para o modelo $S$ (veja Tabela 5.4).

Com relação aos modelos $S 1, S 2$ e $S 3$, os valores estimados apresentam comportamentos diferentes para as duas simulações feitas (veja Tabelas 5.5, 5.6 e 5.7).

Notemos que nos quatro modelos os valores das estimativas das correlações são altos, o que indica uma possível dependência entre elementos que pertencem ao mesmo cluster. 
Os resultados deste capítulo são novos. A primeira versão foi publicada em Kolev e Paiva (2002a) e a versão mais recente foi apresentada em Kolev e Paiva (2002b). 


\section{Capítulo 6}

\section{Conclusões e trabalhos futuros}

Os resultados desta tese de doutorado são relativos a somas aleatórias no caso em que as variáveis envolvidas são igualmente correlacionadas. Aplicamos estes resultados novos em análise de risco e séries temporais discretas.

1. Usamos uma forma especial da função geradora de probabilidade do vetor aleatório com as mesmas distribuições marginais. Condicionamos ao caso da existência de variáveis aleatórias igualmente correlacionadas. Estendemos o resultado clássico e como conseqüência deste estudo foi obtida a distribuição exata da soma aleatória, veja (2.8).

2. Mostramos que, sob certas condições, os modelos correspondentes aos riscos dependentes são modelos de super-dispersão relativos ao modelo de independência; veja capítulo 3 .

3. Utilizamos os resultados anteriores e estendemos dois processos (o processo INAR(1) e duas versões do processo DAR(1)). Por meio de simulações, obtemos que os valores estimados da correlação destes processos são positivos; 
veja capítulo 4 .

4. Apresentamos um modelo que considera sinistros distribuídos em clusters independentes e assumimos que existe dependência só entre sinistros que pertencem ao mesmo cluster. Achamos as estimativas dos parâmetros envolvidos no caso particular de dois clusters.

Algumas sugestões de tópicos para a continuidade deste trabalho são as seguintes:

- Considerar que a matriz de variâncias e covariâncias entre as variáveis aleatórias seja do tipo $\rho_{i j}=\rho^{|i-j|}$, para $i \neq j$.

- Estender o conceito de equicorrelação ao conceito de comonotonicidade, utilizando a técnica proposta por Wang (1998), Wang e Dhaene (1998).

- Usar outras medidas de dependência como a cópula e o coeficiente de Gini, para medir a associação de outros tipos de seqüências dependentes de variáveis aleatórias. 


\section{Apêndice A}

\section{Derivadas do capítulo 4}

\section{Processo CINAR(1)}

A seguir apresentamos as derivadas parciais necessárias para calcular as estimativas do modelo CINAR(1) pelo método de máxima verossimilhança, as quais foram fornecidas para efetuar a simulação.

$$
\begin{aligned}
\frac{\partial \mathbb{P}_{\theta}}{\partial \alpha}= & (1-\rho) e^{-\lambda} \sum_{j=0}^{\min \left\{x_{t-1}, x_{t}\right\}} \frac{\lambda^{x_{t}-j}}{\left(x_{t}-j\right) !}\left(\begin{array}{c}
x_{t-1} \\
j
\end{array}\right)\left[\alpha^{j-1}(1-\alpha)^{x_{t-1}-j-1}\left(j-\alpha x_{t-1}\right)\right] \\
& -\rho e^{-\lambda} \frac{\lambda^{x_{t}}}{x_{t} !}+\rho e^{-\lambda} \frac{\lambda^{x_{t}-x_{t-1}}}{\left(x_{t}-x_{t-1}\right) !} \mathbf{1}\left\{x_{t} \geq x_{t-1}\right\} ; \\
\frac{\partial \mathbb{P}_{\theta}}{\partial \rho}= & \left.-e^{-\lambda} \sum_{j=0}^{\min \left\{x_{t-1}, x_{t}\right\}} \frac{\lambda^{x_{t}-j}}{\left(x_{t}-j\right) !}\left(\begin{array}{c}
x_{t-1} \\
j
\end{array}\right) \alpha^{j}(1-\alpha)^{x_{t-1}-j}\right] \\
& +e^{-\lambda}(1-\alpha) \frac{\lambda^{x_{t}}}{x_{t} !}+e^{-\lambda} \alpha \frac{\lambda^{x_{t}-x_{t-1}}}{\left(x_{t}-x_{t-1}\right) !} 1\left\{x_{t} \geq x_{t-1}\right\} ; \\
\frac{\partial \mathbb{P}_{\theta}}{\partial \lambda}= & \left.(1-\rho) \frac{e^{-\lambda}}{\lambda} \sum_{j=0}^{\min \left\{x_{t-1}, x_{t}\right\}} \frac{\lambda^{x_{t}-j}}{\left(x_{t}-j\right) !}\left(\begin{array}{c}
x_{t-1} \\
j
\end{array}\right) \alpha^{j}(1-\alpha)^{x_{t-1}-j}\left(x_{t}-j-1\right)\right] \\
& +\rho \frac{e^{-\lambda}}{\lambda} \frac{\lambda^{x_{t}}}{x_{t} !}(1-\alpha)\left(x_{t}-\lambda\right)+\rho \frac{e^{-\lambda}}{\lambda} \alpha\left(x_{t}-x_{t-1}-\lambda\right) \frac{\lambda^{x_{t}-x_{t-1}}}{\left(x_{t}-x_{t-1}\right) !} \mathbf{1}\left\{x_{t} \geq x_{t-1}\right\} ;
\end{aligned}
$$




$$
\begin{aligned}
& \frac{\partial^{2} \mathbb{P}_{\theta}}{\partial \alpha^{2}}=(1-\rho) e^{-\lambda} \sum_{j=0}^{\min \left\{x_{t-1}, x_{t}\right\}} \frac{\lambda^{x_{t}-j}}{\left(x_{t}-j\right) !}\left(\begin{array}{c}
x_{t-1} \\
j
\end{array}\right) \alpha^{j-2}(1-\alpha)^{x_{t-1}-j-2} \\
& {\left[\left(j-\alpha x_{t-1}\right)\left(j-\alpha x_{t-1}+\alpha\right)-j(1-\alpha)\right] \text {; }} \\
& \frac{\partial^{2} \mathbb{P}_{\theta}}{\partial \rho^{2}}=0 \\
& \frac{\partial^{2} \mathbb{P}_{\theta}}{\partial \lambda^{2}}=(1-\rho) \frac{e^{-\lambda}}{\lambda^{2}} \sum_{j=0}^{\min \left\{x_{t-1}, x_{t}\right\}} \frac{\lambda^{x_{t}-j}}{\left(x_{t}-j\right) !}\left(\begin{array}{c}
x_{t-1} \\
j
\end{array}\right) \alpha^{j}(1-\alpha)^{x_{t-1}-j} \times \\
& \times\left[\left(x_{t}-j-\lambda\right)^{2}+j-x_{t}\right] \\
& +\rho(1-\alpha) \frac{e^{-\lambda}}{\lambda^{2}} \frac{\lambda^{x_{t}}}{x_{t} !}\left[x_{t}\left(x_{t}-1\right)-2 \lambda x_{t}+\lambda^{2}\right] \\
& +\rho \frac{e^{-\lambda}}{\lambda^{2}} \frac{\lambda^{x_{t}-x_{t-1}}}{\left(x_{t}-x_{t-1}\right) !}\left[\left(x_{t}-x_{t-1}\right)\left(x_{t}-x_{t-1}-1\right)-2 \lambda\left(x_{t}-x_{t-1}\right)+\lambda^{2}\right] 1\left\{x_{t} \geq x_{t-1}\right\} ; \\
& \frac{\partial^{2} \mathbb{P}_{\theta}}{\partial \rho \partial \alpha}=-e^{-\lambda} \sum_{j=0}^{\min \left\{x_{t-1}, x_{t}\right\}} \frac{\lambda^{x_{t}-j}}{\left(x_{t}-j\right) !}\left(\begin{array}{c}
x_{t-1} \\
j
\end{array}\right) \alpha^{j-1}(1-\alpha)^{x_{t-1}-j-1}\left[\left(j-\alpha x_{t-1}\right)\right] \\
& -e^{-\lambda} \frac{\lambda^{x_{t}}}{x_{t} !}+e^{-\lambda} \frac{\lambda^{x_{t}-x_{t-1}}}{\left(x_{t}-x_{t-1}\right) !} \mathbf{1}\left\{x_{t} \geq x_{t-1}\right\} \\
& \left.\frac{\partial^{2} \mathbb{P}_{\theta}}{\partial \rho \partial \lambda}=-\frac{e^{-\lambda}}{\lambda} \sum_{j=0}^{\min \left\{x_{t-1}, x_{t}\right\}} \frac{\lambda^{x_{t}-j}}{\left(x_{t}-j\right) !}\left(\begin{array}{c}
x_{t-1} \\
j
\end{array}\right) \alpha^{j}(1-\alpha)^{x_{t-1}-j}\left(x_{t}-j-\lambda\right)\right] \\
& +(1-\alpha) \frac{e^{-\lambda}}{\lambda} \frac{\lambda^{x_{t}}}{x_{t} !}\left(x_{t}-\lambda\right)+\alpha \frac{e^{-\lambda}}{\lambda}\left(x_{t}-x_{t-1}-\lambda\right) \frac{\lambda^{x_{t}-x_{t-1}}}{\left(x_{t}-x_{t-1}\right) !} \mathbf{1}\left\{x_{t} \geq x_{t-1}\right\} ; \\
& \frac{\partial^{2} \mathbb{P}_{\theta}}{\partial \alpha \partial \lambda}=(1-\rho) \frac{e^{-\lambda}}{\lambda} \sum_{j=0}^{\min \left\{x_{t-1}, x_{t}\right\}} \frac{\lambda^{x_{t}-j}}{\left(x_{t}-j\right) !}\left(\begin{array}{c}
x_{t-1} \\
j
\end{array}\right) \alpha^{j-1}(1-\alpha)^{x_{t-1}-j-1} \times \\
& \times\left[\left(j-\alpha x_{t-1}\right)\left(x_{t}-j-\lambda\right)\right] \\
& -\rho \frac{e^{-\lambda}}{\lambda} \frac{\lambda^{x_{t}}}{x_{t} !}\left(x_{t}-\lambda\right)+\rho \frac{e^{-\lambda}}{\lambda} \frac{\lambda^{x_{t}-x_{t-1}}}{\left(x_{t}-x_{t-1}\right) !}\left(x_{t}-x_{t-1}-\lambda\right) 1\left\{x_{t} \geq x_{t-1}\right\} \text {. }
\end{aligned}
$$




\section{Processo DAR(1) estendido}

De forma analoga foi necessário calcular as derivadas parciais para os modelos DAR(1) estendidos. A seguir apresentamos as derivadas parciais para o modelo DAR(1) com binomial thining correlacionado, i.e., Modelo (4.12)

$$
\begin{aligned}
& \frac{\partial \mathbb{P}_{\theta}}{\partial \lambda}=(1-r \alpha) e^{-\beta} \sum_{t=2}^{N}\left[-\frac{\beta^{x_{t}}}{x_{t} !}+\frac{\beta^{x_{t}-1}}{\left(x_{t}-1\right) !}\right] \\
& \frac{\partial \mathbb{P}_{\theta}}{\partial r}=e^{-\beta} \frac{\lambda(1-\alpha)}{1-r} \sum_{t=2}^{N}\left[-\frac{\beta^{x_{t}}}{x_{t} !}+\frac{\beta^{x_{t}-1}}{\left(x_{t}-1\right) !}\right]-e^{-\beta} \sum_{t=2}^{N} \frac{\beta^{x_{t}}}{x_{t} !} \\
& +\sum_{t=2}^{N}\left(\begin{array}{c}
x_{t-1} \\
x_{t}
\end{array}\right) \alpha^{x_{t}}(1-\alpha)^{x_{t-1}-x_{t}} 1\left\{x_{t} \leq x_{t-1}\right\} \\
& \frac{\partial \mathbb{P}_{\theta}}{\partial \alpha}=\lambda r e^{-\beta} \sum_{t=2}^{N}\left[-\frac{\beta^{x_{t}}}{x_{t} !}+\frac{\beta^{x_{t}-1}}{\left(x_{t}-1\right) !}\right] \\
& +r e^{-\beta} \sum_{t=2}^{N}\left(\begin{array}{c}
x_{t-1} \\
x_{t}
\end{array}\right) \alpha^{x_{t}-1}(1-\alpha)^{x_{t-1}-x_{t-1}}\left(x_{t}-\alpha x_{t-1}\right) 1\left\{x_{t} \leq x_{t-1}\right\} \text {; } \\
& \frac{\partial^{2} \mathbb{P}_{\theta}}{\partial \lambda^{2}}=e^{-\beta} \frac{(-r \alpha)^{2}}{1-r} \sum_{t=2}^{N}\left[-\frac{\beta^{x_{t}}}{x_{t} !}-2 \frac{\beta^{x_{t}-1}}{\left(x_{t}-1\right) !}+\frac{\beta^{x_{t}-2}}{\left(x_{t}-2\right) !}\right] \text {; } \\
& \frac{\partial^{2} \mathbb{P}_{\theta}}{\partial r^{2}}=e^{-\beta} \frac{\lambda^{2}(1-\alpha)^{2}}{(1-r)^{3}} \sum_{t=2}^{N}\left[-\frac{\beta^{x_{t}}}{x_{t} !}-2 \frac{\beta^{x_{t}-1}}{\left(x_{t}-1\right) !}+\frac{\beta^{x_{t}-2}}{\left(x_{t}-2\right) !}\right] \text {; } \\
& \frac{\partial^{2} \mathbb{P}_{\theta}}{\partial \alpha^{2}}=\lambda^{2} r^{2} e^{-\beta} \sum_{t=2}^{N}\left[-\frac{\beta^{x_{t}}}{x_{t} !}-2 \frac{\beta^{x_{t}-1}}{\left(x_{t}-1\right) !}+\frac{\beta^{x_{t}-2}}{\left(x_{t}-2\right) !}\right] \\
& +r e^{-\beta} \sum_{t=2}^{N}\left(\begin{array}{c}
x_{t-1} \\
x_{t}
\end{array}\right)\left[\alpha^{x_{t}-2}(1-\alpha)^{x_{t-1}-x_{t}-2}\right. \\
& \left.\left\{\left(x_{t}-\alpha x_{t-1}\right)^{2}+\alpha\left(x_{t}-\alpha x_{t-1}\right)-x_{t}(1-\alpha)\right\}\right] 1\left\{x_{t} \leq x_{t-1}\right\} ; \\
& \frac{\partial^{2} \mathbb{P}_{\theta}}{\partial \lambda \partial \alpha}=r e^{-\beta} \sum_{t=2}^{N}\left[-\frac{\beta^{x_{t}}}{x_{t} !}+\frac{\beta^{x_{t}-1}}{\left(x_{t}-1\right) !}\right] \\
& +\lambda r e^{-\beta} \frac{1-r \alpha}{1-r} \sum_{t=2}^{N}\left[-\frac{\beta^{x_{t}}}{x_{t} !}+\frac{\beta^{x_{t}-1}}{\left(x_{t}-1\right) !}\right] \text {; }
\end{aligned}
$$




$$
\begin{aligned}
\frac{\partial^{2} \mathbb{P}_{\theta}}{\partial \lambda \partial r}= & \alpha e^{-\beta} \sum_{t=2}^{N}\left[\frac{\beta^{x_{t}}}{x_{t} !}-\frac{\beta^{x_{t}-1}}{\left(x_{t}-1\right) !}\right] \\
& +e^{-\beta} \frac{\lambda(1-\alpha)}{1-r} \frac{1-r \alpha}{1-r} \sum_{t=2}^{N}\left[\frac{\beta^{x_{t}}}{x_{t} !}-2 \frac{\beta^{x_{t}-1}}{\left(x_{t}-1\right) !}+\frac{\beta^{x_{t}-2}}{\left(x_{t}-2\right) !}\right] \\
\frac{\partial^{2} \mathbb{P}_{\theta}}{\partial \alpha \partial r}= & \lambda e^{-\beta} \sum_{t=2}^{N}\left[\frac{\beta^{x_{t}}}{x_{t} !}-\frac{\beta^{x_{t}-1}}{\left(x_{t}-1\right) !}\right] \\
& +\lambda r e^{-\beta} \frac{\lambda(1-\alpha)}{(1-r)^{2}} \sum_{t=2}^{N}\left[-\frac{\beta^{x_{t}}}{x_{t} !}+2 \frac{\beta^{x_{t}-1}}{\left(x_{t}-1\right) !}-\frac{\beta^{x_{t}-2}}{\left(x_{t}-2\right) !}\right] \\
& +\sum_{t=2}^{N}\left(\begin{array}{c}
x_{t-1} \\
x_{t}
\end{array}\right) \alpha^{x_{t}-1}(1-\alpha)^{x_{t-1}-x_{t}-1}\left(x_{t}-\alpha x_{t-1}\right) 1\left\{x_{t} \leq x_{t-1}\right\} .
\end{aligned}
$$




\section{Apêndice B}

\section{Programas do capítulo 4}

Neste apêndice apresentamos o programa feito na linguagem de programação Ox (veja Doornik, 1996). Este programa apresenta as estimativas dos parâmetros pelo método de máxima verossimilhança aplicando o método de Newton-Rhapson do processo CINAR(1). Foram fornecidas os valores iniciais encontrados pelo método dos momentos.

Os dados resp97.txt são os relativos aos pacientes com doença respiratória no período de 1997. 
A seguir a notação usada ao longo deste programa

$$
\begin{aligned}
& \text { soma1 }=\sum_{i=1}^{n} x_{t} x_{t-1} \quad \text { factXt1 }=x_{t-1} \text { ! } \\
& \text { soma } 2=\sum_{i=1}^{n} x_{t} \quad \text { factXt2 }=\left|x_{t}-j\right| \text { ! } \\
& \text { soma3 }=\sum_{i=1}^{n} x_{t-1} \quad \text { factXt3 }=\left(\left|x_{t-1}-j\right|\right) \text { ! } \\
& \text { soma4 }=\sum_{i=1}^{n} x_{t-1}^{2} \quad \text { factXt4 }=j \text { ! } \\
& \alpha_{0}=\frac{\sum_{i=1}^{n} x_{t} x_{t-1}-\frac{\sum_{i=1}^{n} x_{t} \sum_{i=1}^{n} x_{t-1}}{n}}{\sum_{i=1}^{n} x_{t-1}^{2}-\frac{\left(\sum_{i=1}^{n} x_{t-1}\right)^{2}}{n}} \quad \text { factXt5 }=\left|x_{t}-x_{t-1}\right| ! \\
& \lambda_{0}=\frac{\sum_{i=1}^{n} x_{t}-\alpha_{0} \sum_{i=1}^{n} x_{t-1}}{n} \quad \text { factXt6 }=x_{t} \text { ! } \\
& A=\lambda_{0}^{x_{t}-j} \quad B 1=\alpha_{0}^{j-2}\left(1-\alpha_{0}\right)^{x_{t}-j-2} \\
& B=\alpha_{0}^{j-1}\left(1-\alpha_{0}\right)^{x_{t}-j-1}\left(j-\alpha_{0} x_{t-1}\right) \quad B 2=\left(j-\alpha_{0} x_{t-1}\right)\left(j--\alpha_{0} x_{t-1}+\alpha_{0}\right)-j\left(1-\alpha_{0}\right) \\
& C=\lambda_{0}^{x_{t}} \quad B 3=B 1 * B 2 \\
& J=\left(\begin{array}{c}
x_{t-1} \\
j
\end{array}\right) \frac{1}{\left(x_{t}-j\right) !} \quad E 2=\frac{e^{-\lambda_{0}}}{\lambda_{0}^{2}} \\
& D=\lambda_{0}^{x_{t}-x_{t}-1} \quad Q 1=\left(x_{t}-j\right)\left(x_{t}-j-1\right) \\
& E=e^{-\lambda_{0}} \quad Q 2=2 \lambda_{0}\left(x_{t}-j\right) \\
& G=A * J * B \quad Q 3=\lambda_{0}^{2} \\
& H=\frac{C}{x_{t} !} \quad Q=Q 1-Q 2+Q 3 \\
& W I=\frac{D}{\left|x_{t}-x_{t-1}\right| !} \quad R 1=x_{t}\left(x_{t}-1\right) \\
& \text { nresult }=A * J * B \quad R 2=2 \lambda_{0} x_{t} \\
& \text { dresult }=G+H-W I \quad R=R 1-R 2+Q 3 \\
& \operatorname{minimo}=\min \left\{x_{t}, x_{t-1}\right\} \quad S 1=\left(x_{t}-x_{t-1}\right)\left(x_{t}-x_{t-1}-1\right) \\
& K=\alpha_{0}^{j}\left(1-\alpha_{0}\right)^{x_{t-1}-j} \quad S 2=2 \lambda_{0}\left(x_{t}-x_{t-1}\right) \\
& L=x_{t-j}-\lambda_{0} \quad S=S 1-S 2+Q 3 \\
& E 1=\frac{e^{-\lambda_{0}}}{\lambda_{0}} \quad W W I-\rho_{0} E 2 * D * S \\
& M=x_{t}-\lambda_{0} \quad W W Q=\alpha_{0} * D * E 1 * P \\
& P=x_{t}-x_{t-1}-\lambda_{0} \quad W W W I=\frac{W W Q}{\left(x_{t}-x_{t-1}\right) !} \\
& \text { prob0 }=\mathbb{P}_{\theta}\left(x_{t-1}, x_{t}\right) \quad \mathrm{d} 2 \mathrm{rho} 0=\frac{\partial^{2} \boldsymbol{P}_{\theta}}{\partial \rho^{2}} \\
& \mathrm{~d} 1 \mathrm{alpha} 0=\frac{\partial \boldsymbol{P}_{\theta}}{\partial \alpha} \quad \mathrm{d} 2 \text { lambda0 } 0=\frac{\partial^{2} \boldsymbol{P}_{\theta}}{\partial \lambda^{2}} \\
& \mathrm{~d} 1 \mathrm{rho} 0=\frac{\partial \boldsymbol{P}_{\theta}}{\partial \rho} \quad \mathrm{d} 2 \text { rhoalpha0 }=\frac{\partial^{2} \boldsymbol{P}_{\theta}}{\partial \rho \partial \alpha} \\
& \text { d1lambda0 }=\frac{\partial \boldsymbol{P}_{\theta}}{\partial \lambda} \quad \mathrm{d} 2 \text { rholambda0 }=\frac{\partial^{2} \boldsymbol{P}_{\theta}}{\partial \rho \partial \lambda} \\
& \text { d2alpha0 }=\frac{\partial^{2} \boldsymbol{P}_{\theta}}{\partial \alpha^{2}} \quad \text { d2alphalambda0 }=\frac{\partial^{2} \boldsymbol{P}_{\theta}}{\partial \alpha \partial \lambda}
\end{aligned}
$$




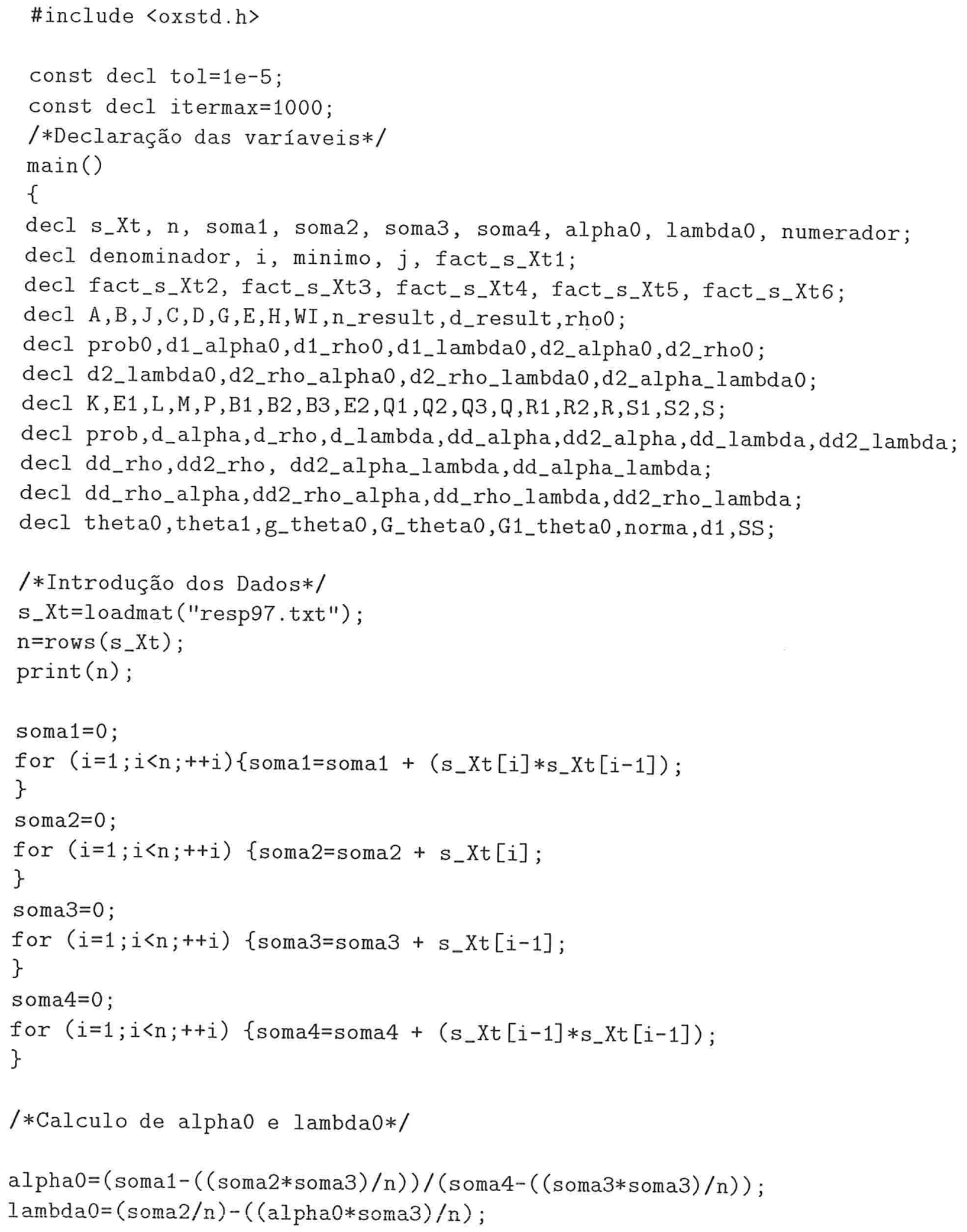




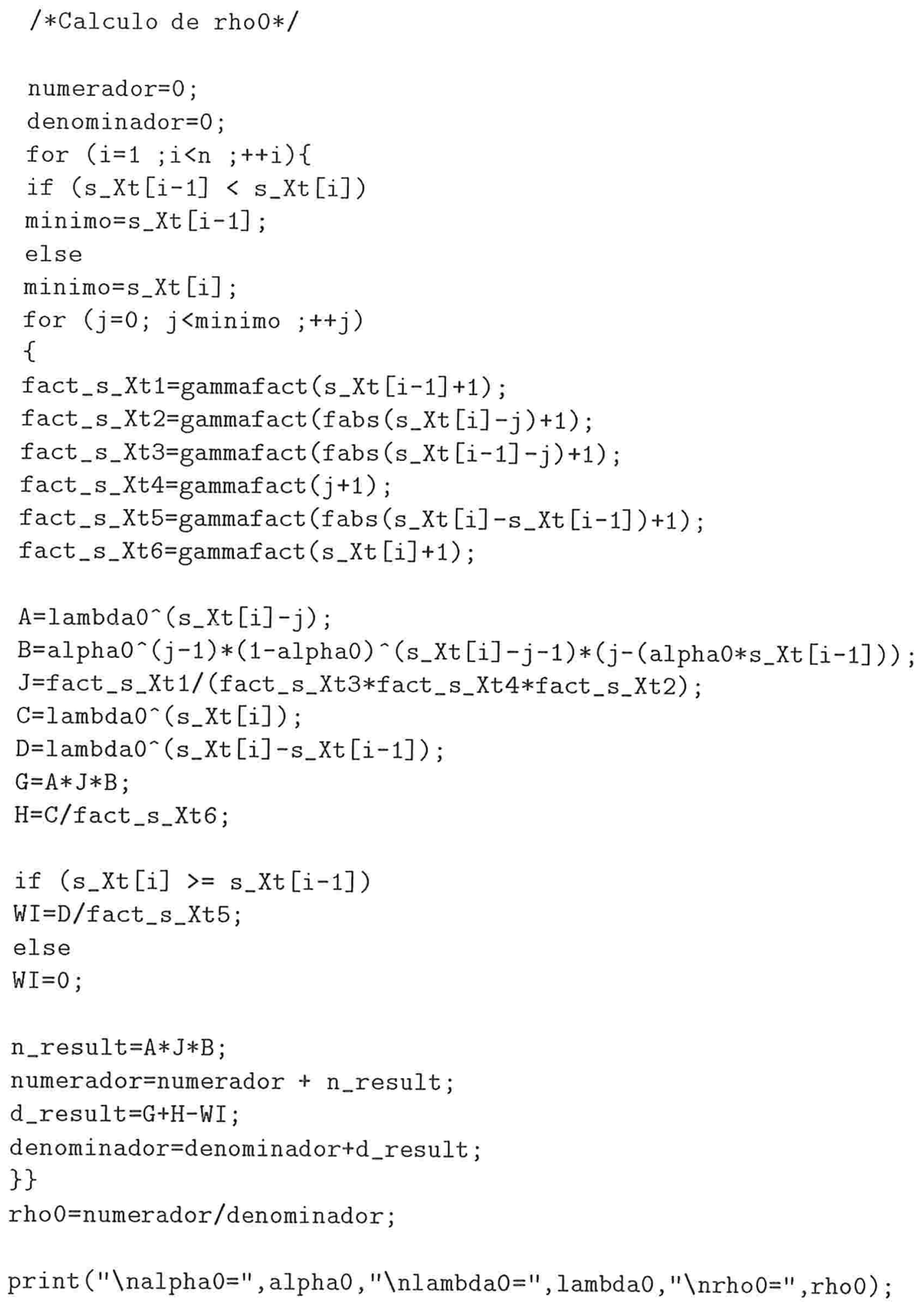




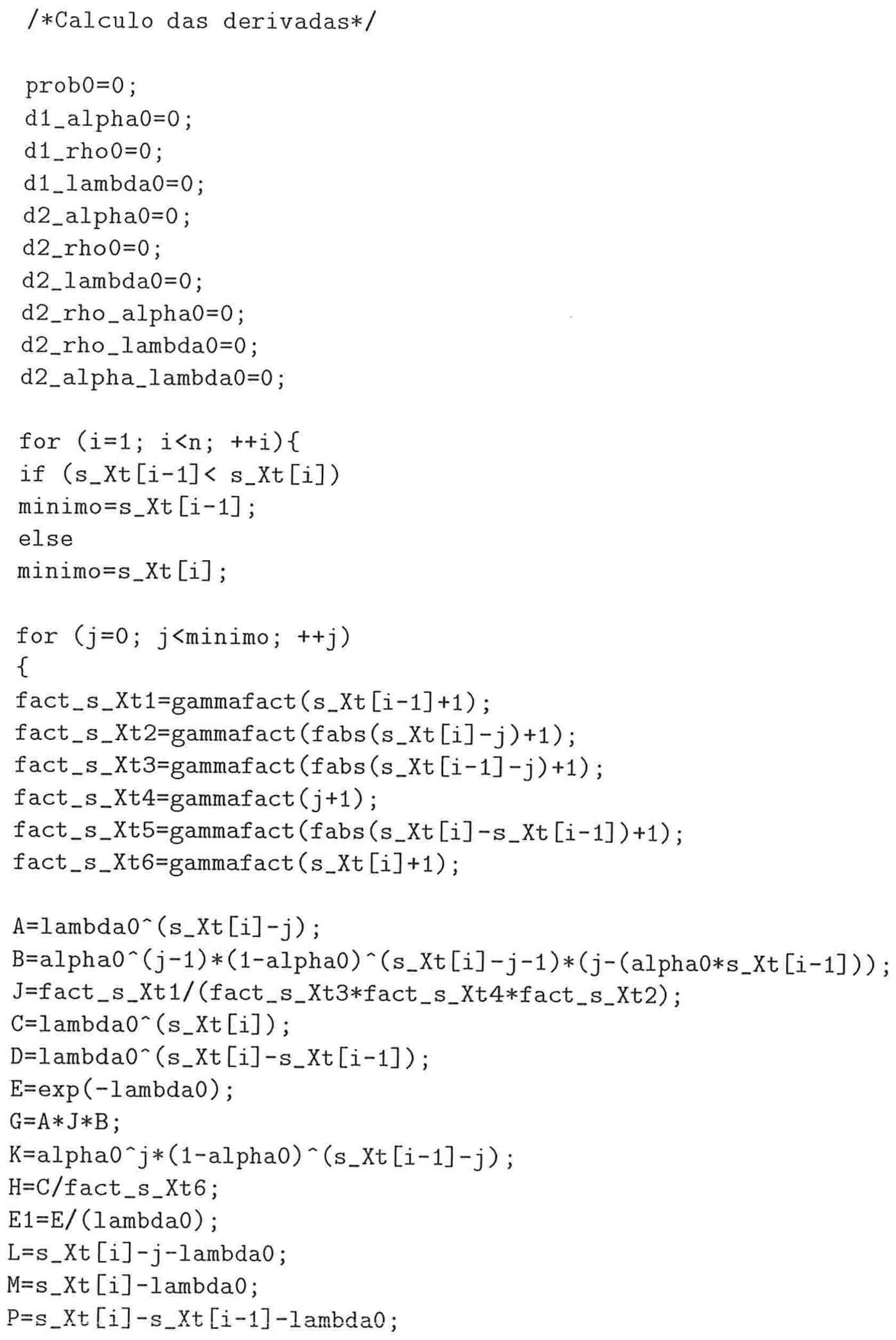




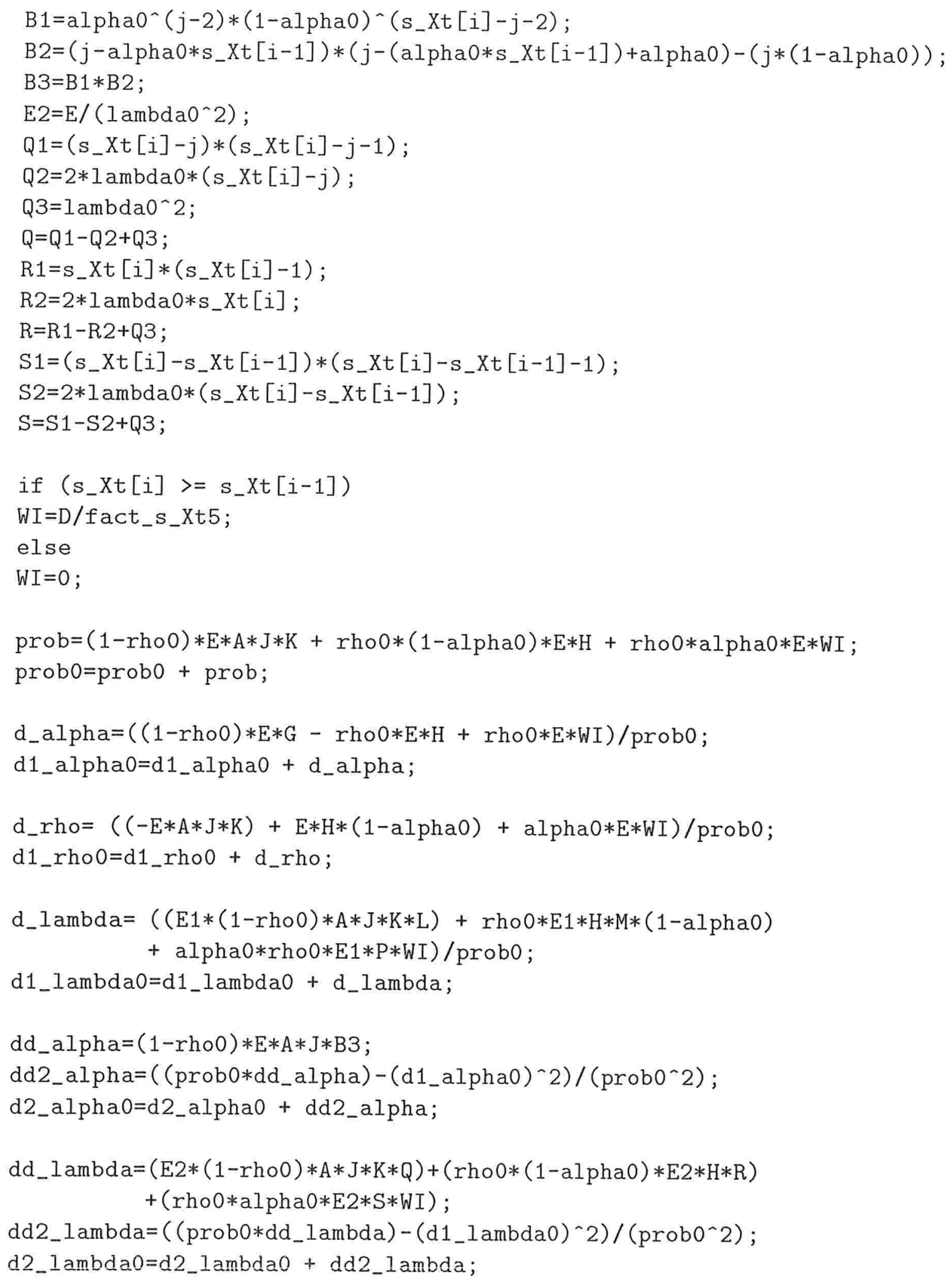




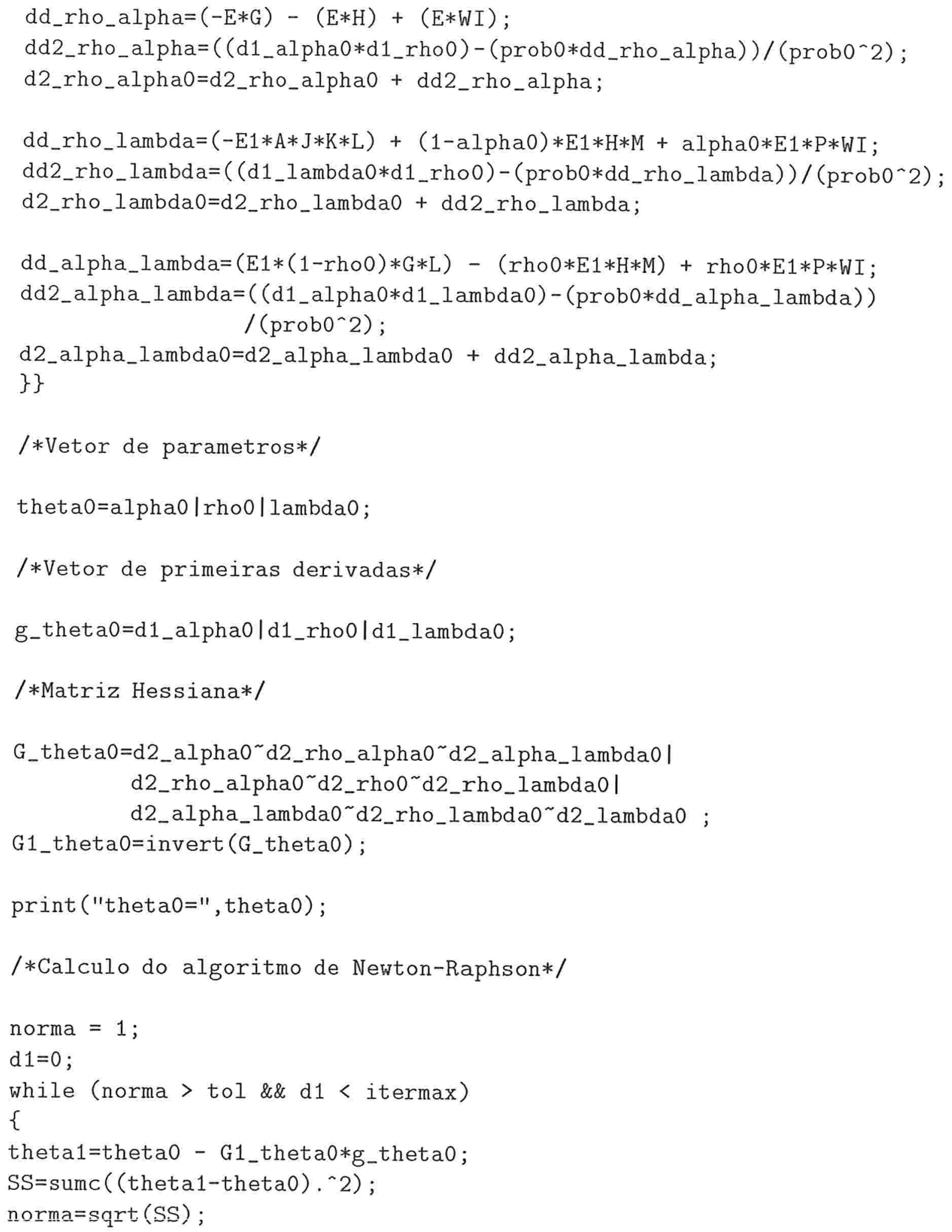




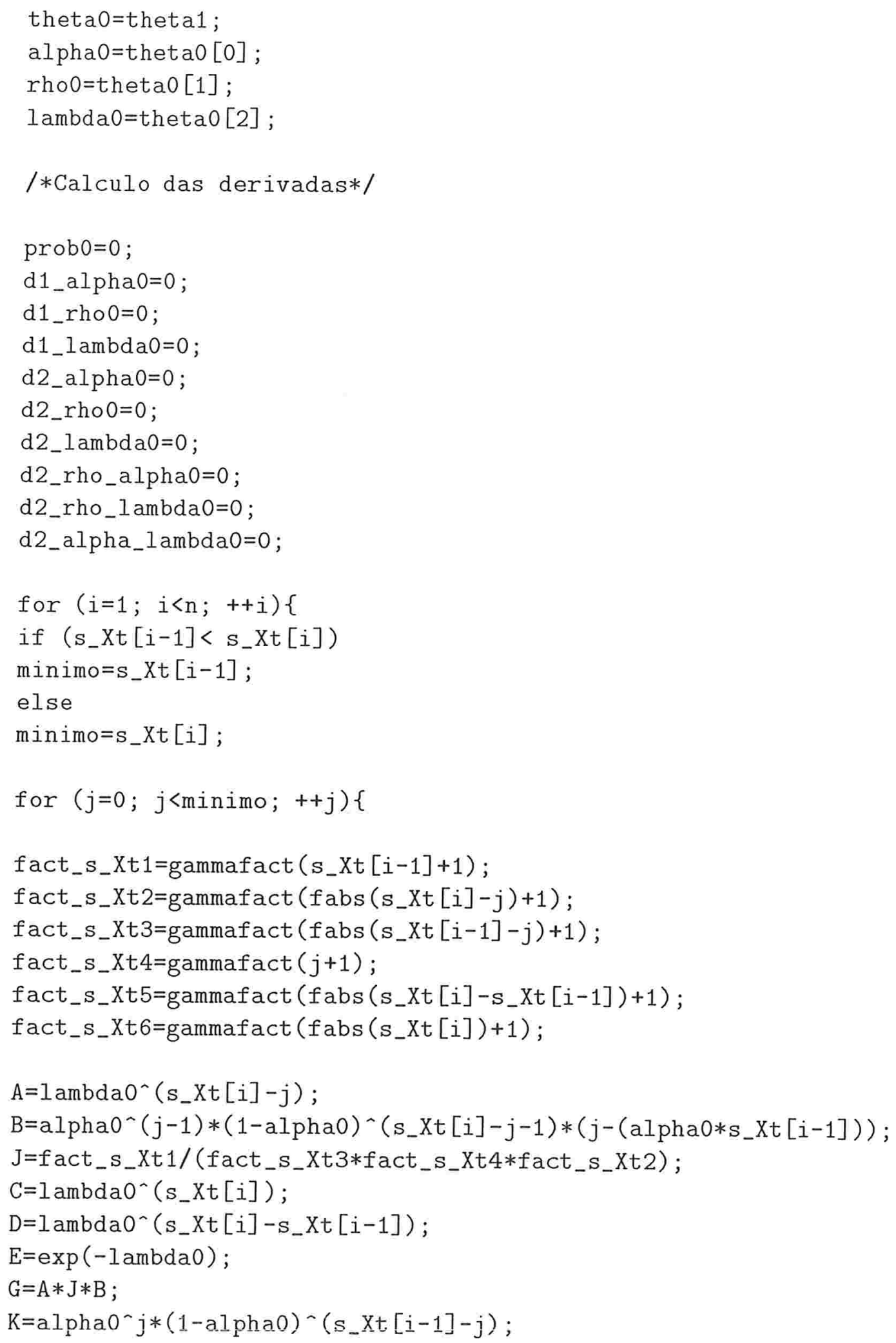




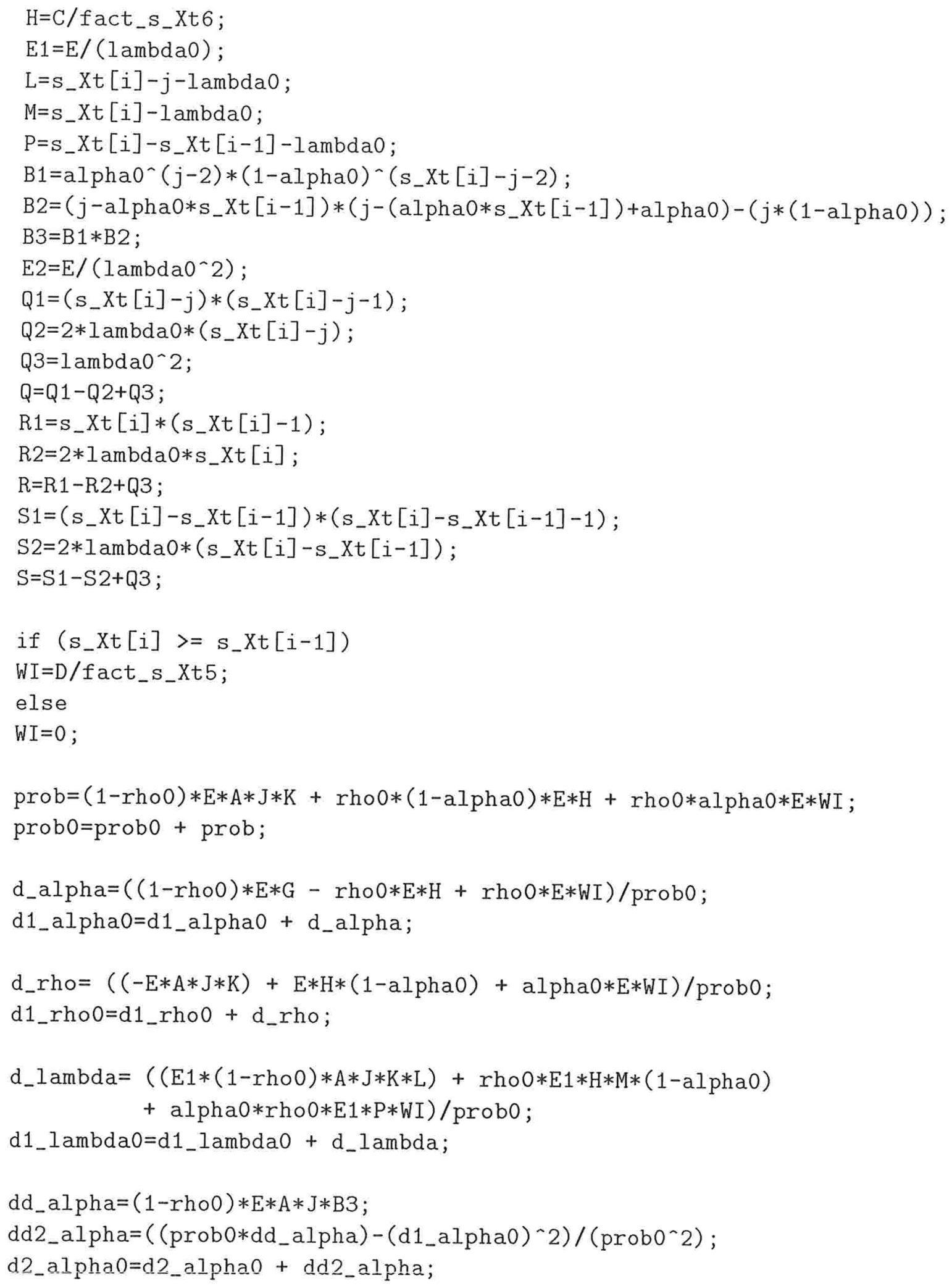




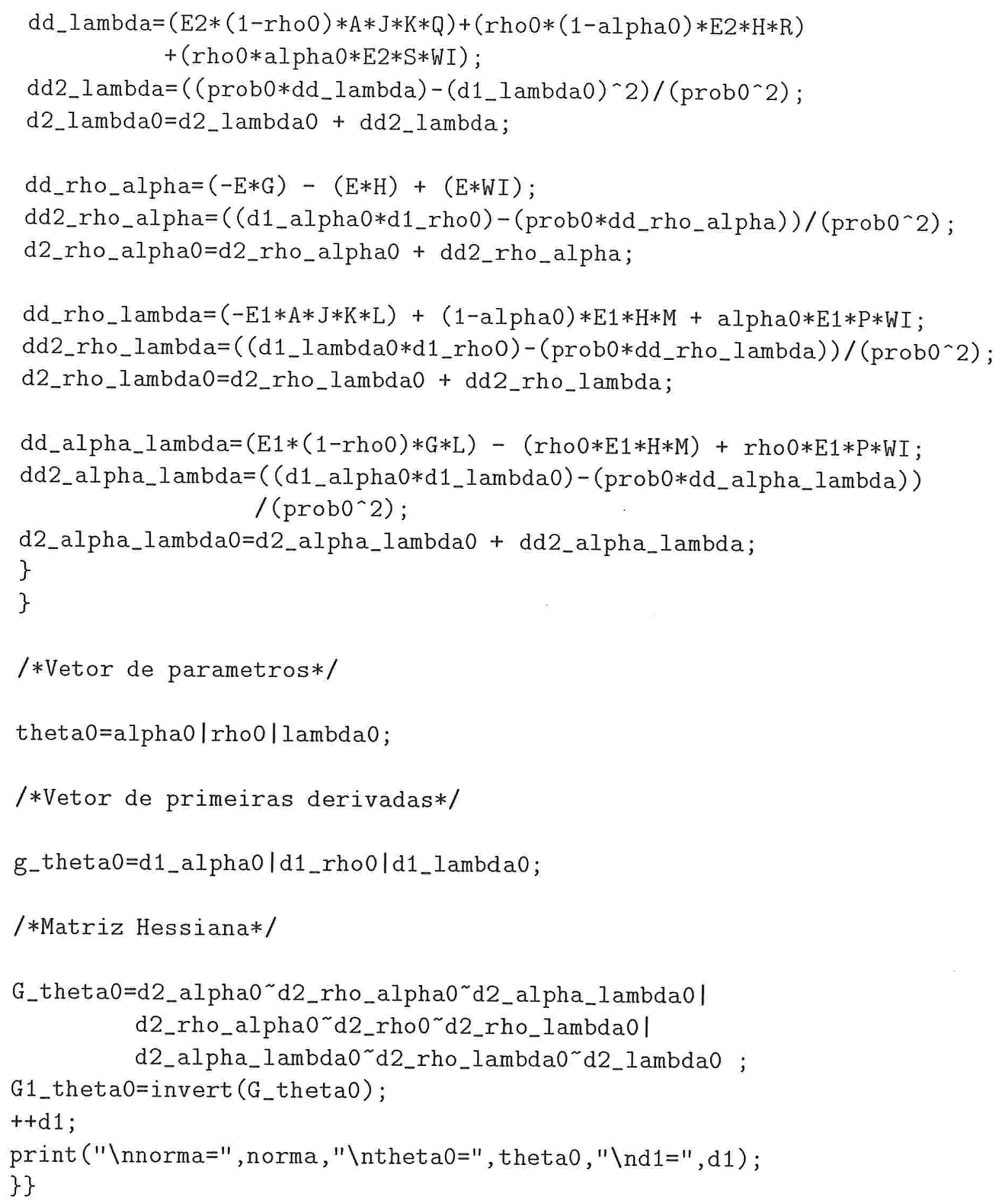




\section{Apêndice $\mathrm{C}$}

\section{Derivadas do capítulo 5}

Neste apêndice apresentamos as equações normais do modelo S.

Seja $f=\mathbb{P}\left(S_{n}=n_{1}\right)$, dado em (5.4) A igualdade $0=\frac{\partial f}{\partial p_{1}}$ é equivalente a

$$
\begin{aligned}
0= & -\rho_{1} \rho_{2} \alpha_{2}\left(1-\alpha_{1}\right)\left(\begin{array}{c}
N \\
n_{1}
\end{array}\right)\left(1-p_{1}\right)^{n_{1}-1} p_{1}^{N-n_{1}-1}\left(n_{1}-N\left(1-p_{1}\right)\right) \\
& +\rho_{1} \rho_{2} \alpha_{1}\left(1-\alpha_{2}\right)\left(\begin{array}{c}
N \\
n_{1}
\end{array}\right) p_{1}^{n_{1}-1}\left(1-p_{1}\right)^{N-n_{1} 1}\left(n_{1}-N p_{1}\right) \\
& -\rho_{1}\left(1-\rho_{2}\right)\left(1-\alpha_{1}\right)\left(\begin{array}{c}
N \\
n_{1}
\end{array}\right)\left(\alpha_{2}-\alpha_{2} p_{1}\right)^{n_{1}-1}\left(1-\alpha_{2}+\alpha_{2} p_{1}\right)^{N-n_{1}-1} \times \\
& \times \alpha_{2}\left(n_{1}-N\left(\alpha_{2}-\alpha_{2} p_{1}\right)\right) \\
& +\left(1-\rho_{1}\right) \rho_{2}\left(1-\alpha_{2}\right)\left(\begin{array}{c}
N \\
n_{1}
\end{array}\right)\left(\alpha_{1} p_{1}\right)^{n_{1}-1}\left(1-\alpha_{1} p_{1}\right)^{N-n_{1}-1} \alpha_{1}\left(n_{1}-N \alpha_{1} p_{1}\right) \\
& +\rho_{1}\left(1-\rho_{2}\right) \alpha_{1}\left(\begin{array}{c}
N \\
n_{1}
\end{array}\right)\left(\alpha_{2}+p_{1}-\alpha_{2} p_{1}\right)^{n_{1}-1}\left(1-\alpha_{2}-p_{1}+\alpha_{2} p_{1}\right)^{N-n_{1}-1} \times \\
& \times\left(1-\alpha_{2}\right)\left(n_{1}-N\left(\alpha_{2}+p_{1}-\alpha_{2} p_{1}\right)\right) \\
& +\left(1-\rho_{1}\right) \rho_{2} \alpha_{2}\left(\begin{array}{c}
N \\
n_{1}
\end{array}\right)\left(1-\alpha_{1}+\alpha_{1} p_{1}\right)^{n_{1}-1}\left(\alpha_{1}-\alpha_{1} p_{1}\right)^{N-n_{1}-1} \times \\
& \times \alpha_{1}\left(n_{1}-N\left(1-\alpha_{1}+\alpha_{1} p_{1}\right)\right) \\
& -\left(1-\rho_{1}\right)\left(1-\rho_{2}\right)\left(\begin{array}{c}
N \\
n_{1}
\end{array}\right)\left(\alpha_{2}-\alpha_{2} p_{1}+\alpha_{1} p_{1}\right)^{n_{1}-1}\left(1-\alpha_{2}+\alpha_{2} p_{1}-\alpha_{1} p_{1}\right)^{N-n_{1}-1} \times \\
& \times\left(\alpha_{1}-\alpha_{2}\right)\left(n_{1}-N\left(\alpha_{2}-\alpha_{2} p_{1}+\alpha_{1} p_{1}\right)\right) .
\end{aligned}
$$


Para $0=\frac{\partial f}{\partial \alpha_{1}}$ obtemos a igualdade

$$
\begin{aligned}
0= & -\rho_{1} \rho_{2}\left(1-\alpha_{2}\right) \mathbf{1}\left\{n_{1}=0\right\}+\rho_{1} \rho_{2} \alpha_{2} 1\left\{n_{1}=N\right\}-\rho_{1} \rho_{2} \alpha_{2}\left(\begin{array}{c}
N \\
n_{1}
\end{array}\right)\left(1-p_{1}\right)_{1}^{n} p_{1}^{N-n_{1}} \\
& +\rho_{1} \rho_{2}\left(1-\alpha_{2}\right)\left(\begin{array}{c}
N \\
n_{1}
\end{array}\right) p_{1}^{n_{1}}\left(1-p_{1}\right)^{N-n_{1}} \\
& -\rho_{1}\left(1-\rho_{2}\right)\left(\begin{array}{c}
N \\
n_{1}
\end{array}\right)\left(\alpha_{2}-\alpha_{2} p_{1}\right)_{1}^{n}\left(1-\alpha_{2}+\alpha_{2} p_{1}\right)^{N-n_{1}} \\
& +\left(1-\rho_{1}\right) \rho_{2}\left(1-\alpha_{2}\right)\left(\begin{array}{c}
N \\
n_{1}
\end{array}\right)\left(\alpha_{1} p_{1}\right)^{n_{1}-1}\left(1-\alpha_{1} p_{1}\right)^{N-n_{1}-1} p_{1}\left(n_{1}-N \alpha_{1} p_{1}\right) \\
& +\rho_{1}\left(1-\rho_{2}\right)\left(\begin{array}{c}
N \\
n_{1}
\end{array}\right)\left(\alpha_{2}+p_{1}-\alpha_{2} p_{1}\right)^{n_{1}}\left(1-\alpha_{2}-p_{1}+\alpha_{2} p_{1}\right)^{N-n_{1}} \\
& -\left(1-\rho_{1}\right) \rho_{2} \alpha_{2}\left(\begin{array}{c}
N \\
n_{1}
\end{array}\right)\left(1-\alpha_{1}+\alpha_{1} p_{1}\right)^{n_{1}-1}\left(\alpha_{1}-\alpha_{1} p_{1}\right)^{N-n_{1}-1} \times \\
& \times\left(1-p_{1}\right)\left(n_{1}-N\left(1-\alpha_{1}+\alpha_{1} p_{1}\right)\right) \\
& +\left(1-\rho_{1}\right)\left(1-\rho_{2}\right)\left(\begin{array}{c}
N \\
n_{1}
\end{array}\right)\left(\alpha_{2}-\alpha_{2} p_{1}+\alpha_{1} p_{1}\right)^{n_{1}-1}\left(1-\alpha_{2}+\alpha_{2} p_{1}-\alpha_{1} p_{1}\right)^{N-n_{1}-1} \times \\
& \times p_{1}\left(n_{1}-N\left(\alpha_{2}-\alpha_{2} p_{1}+\alpha_{1} p_{1}\right)\right) .
\end{aligned}
$$

A derivada de $\mathbb{P}\left(S_{n}=n_{1}\right)$ com respecto a $\alpha_{2}$

$$
\begin{aligned}
0= & \frac{\partial f}{\partial \alpha_{2}} \\
= & -\rho_{1} \rho_{2}\left(1-\alpha_{1}\right) 1\left\{n_{1}=0\right\}+\rho_{1} \rho_{2} \alpha_{1} \mathbf{1}\left\{n_{1}=N\right\} \\
& +\rho_{1} \rho_{2}\left(1-\alpha_{1}\right)\left(\begin{array}{c}
N \\
n_{1}
\end{array}\right)\left(1-p_{1}\right)_{1}^{n} p_{1}^{N-n_{1}} \\
& -\rho_{1} \rho_{2} \alpha_{1}\left(\begin{array}{c}
N \\
n_{1}
\end{array}\right) p_{1}^{n_{1}}\left(1-p_{1}\right)^{N-n_{1}}-\left(1-\rho_{1}\right) \rho_{2}\left(\begin{array}{c}
N \\
n_{1}
\end{array}\right)\left(\alpha_{1} p_{1}\right)^{n_{1}}\left(1-\alpha_{1} p_{1}\right)^{N-n_{1}} \\
& +\rho_{1}\left(1-\rho_{2}\right)\left(1-\alpha_{1}\right)\left(\begin{array}{c}
N \\
n_{1}
\end{array}\right)\left(\alpha_{2}-\alpha_{2} p_{1}\right)^{n_{1}-1}\left(1-\alpha_{2}+\alpha_{2} p_{1}\right)^{N-n_{1}-1} \times \\
& \times\left(1-p_{1}\right)\left(n_{1}-N\left(\alpha_{2}-\alpha_{2} p_{1}\right)\right) \\
& +\rho_{1}\left(1-\rho_{2}\right) \alpha_{1}\left(\begin{array}{c}
N \\
n_{1}
\end{array}\right)\left(\alpha_{2}+p_{1}-\alpha_{2} p_{1}\right)^{n_{1}-1}\left(1-\alpha_{2}-p_{1}+\alpha_{2} p_{1}\right)^{N-n_{1}-1} \times \\
& \times\left(1-p_{1}\right)\left(n_{1}-N\left(\alpha_{2}+p_{1}-\alpha_{2} p_{1}\right)\right) \\
& +\left(1-\rho_{1}\right) \rho_{2}\left(\begin{array}{c}
N \\
n_{1}
\end{array}\right)\left(1-\alpha_{1}+\alpha_{1} p_{1}\right)^{n_{1}}\left(\alpha_{1}-\alpha_{1} p_{1}\right)^{N-n_{1}} \\
& +\left(1-\rho_{1}\right)\left(1-\rho_{2}\right)\left(\begin{array}{c}
N \\
n_{1}
\end{array}\right)\left(\alpha_{2}-\alpha_{2} \tilde{p}_{1}+\alpha_{1} \tilde{p}_{1}\right)^{n_{1}-1}\left(1-\alpha_{2}+\alpha_{2} p_{1}-\alpha_{1} p_{1}\right)^{N-n_{1}-1} \times
\end{aligned}
$$




$$
\times\left(1-p_{1}\right)\left(n_{1}-N\left(\alpha_{2}-\alpha_{2} p_{1}+\alpha_{1} p_{1}\right)\right) .
$$

Analogamente $0=\frac{\partial f}{\partial \rho_{1}}$ é equvalente a

$$
\begin{aligned}
0= & \rho_{2}\left(1-\alpha_{1}\right)\left(1-\alpha_{2}\right) 1\left\{n_{1}=0\right\}+\rho_{2} \alpha_{1} \alpha_{2} 1\left\{n_{1}=N\right\} \\
& +\rho_{2} \alpha_{2}\left(1-\alpha_{1}\right)\left(\begin{array}{c}
N \\
n_{1}
\end{array}\right)\left(1-p_{1}\right)_{1}^{n} p_{1}^{N-n_{1}} \\
& +\rho_{2} \alpha_{1}\left(1-\alpha_{2}\right)\left(\begin{array}{c}
N \\
n_{1}
\end{array}\right) p_{1}^{n_{1}}\left(1-p_{1}\right)^{N-n_{1}} \\
& +\left(1-\rho_{2}\right)\left(1-\alpha_{1}\right)\left(\begin{array}{c}
N \\
n_{1}
\end{array}\right)\left(\alpha_{2}-\alpha_{2} p_{1}\right)^{n_{1}}\left(1-\alpha_{2}+\alpha_{2} p_{1}\right)^{N-n_{1}} \\
& -\rho_{2}\left(1-\alpha_{2}\right)\left(\begin{array}{c}
N \\
n_{1}
\end{array}\right)\left(\alpha_{1} p_{1}\right)_{1}^{n}\left(1-\alpha_{1} p_{1}\right)^{N-n_{1}} \\
& +\left(1-\rho_{2}\right) \alpha_{1}\left(\begin{array}{c}
N \\
n_{1}
\end{array}\right)\left(\alpha_{2}+p_{1}-\alpha_{2} p_{1}\right)^{n_{1}}\left(1-\alpha_{2}-p_{1}+\alpha_{2} p_{1}\right)^{N-n_{1}} \\
& -\rho_{2} \alpha_{2}\left(\begin{array}{c}
N \\
n_{1}
\end{array}\right)\left(1-\alpha_{1}+\alpha_{1} p_{1}\right)^{n_{1}}\left(\alpha_{1}-\alpha_{1} p_{1}\right)^{N-n_{1}} \\
& -\left(1-\rho_{2}\right)\left(\begin{array}{c}
N \\
n_{1}
\end{array}\right)\left(\alpha_{2}-\alpha_{2} p_{1}+\alpha_{1} p_{1}\right)^{n_{1}}\left(1-\alpha_{2}+\alpha_{2} p_{1}-\alpha_{1} p_{1}\right)^{N-n_{1}},
\end{aligned}
$$

e $0=\frac{\partial f}{\partial \rho_{2}}$ é equivalente a

$$
\begin{aligned}
0= & \rho_{1}\left(1-\alpha_{1}\right)\left(1-\alpha_{2}\right) 1\left\{n_{1}=0\right\}+\rho_{1} \alpha_{1} \alpha_{2} 1\left\{n_{1}=N\right\} \\
& +\rho_{1} \alpha_{2}\left(1-\alpha_{1}\right)\left(\begin{array}{c}
N \\
n_{1}
\end{array}\right)\left(1-p_{1}\right)_{1}^{n} p_{1}^{N-n_{1}} \\
& +\rho_{1} \alpha_{1}\left(1-\alpha_{2}\right)\left(\begin{array}{c}
N \\
n_{1}
\end{array}\right) p_{1}^{n_{1}}\left(1-p_{1}\right)^{N-n_{1}} \\
& +-r h o_{1}\left(1-\alpha_{1}\right)\left(\begin{array}{c}
N \\
n_{1}
\end{array}\right)\left(\alpha_{2}-\alpha_{2} p_{1}\right)^{n_{1}}\left(1-\alpha_{2}+\alpha_{2} p_{1}\right)^{N-n_{1}} \\
& +\left(1-\rho_{1}\right)\left(1-\alpha_{2}\right)\left(\begin{array}{c}
N \\
n_{1}
\end{array}\right)\left(\alpha_{1} p_{1}\right)_{1}^{n}\left(1-\alpha_{1} p_{1}\right)^{N-n_{1}} \\
& -\rho_{1} \alpha_{1}\left(\begin{array}{c}
N \\
n_{1}
\end{array}\right)\left(\alpha_{2}+p_{1}-\alpha_{2} p_{1}\right)^{n_{1}}\left(1-\alpha_{2}-p_{1}+\alpha_{2} p_{1}\right)^{N-n_{1}} \\
& +\left(1-\rho_{1}\right) \alpha_{2}\left(\begin{array}{c}
N \\
n_{1}
\end{array}\right)\left(1-\alpha_{1}+\alpha_{1} p_{1}\right)^{n_{1}}\left(\alpha_{1}-\alpha_{1} p_{1}\right)^{N-n_{1}} \\
& -\left(1-\rho_{1}\right)\left(\begin{array}{c}
N \\
n_{1}
\end{array}\right)\left(\alpha_{2}-\alpha_{2} p_{1}+\alpha_{1} p_{1}\right)^{n_{1}}\left(1-\alpha_{2}+\alpha_{2} p_{1}-\alpha_{1} p_{1}\right)^{N-n_{1}}
\end{aligned}
$$




\section{Apêndice $\mathbf{D}$}

\section{Programas do capítulo 5}

Apresentamos a seguir o programa desenvolvido na linguagem de programação Ox, (veja Doornik, 1996) usado para a obtenção das estimativas de máxima verossimilhança usando o algoritmo Newton-Rhapson. Este programa é para o caso do modelo S, i.e., considerando $\alpha_{1} \neq \alpha_{2}$ e $\rho_{1} \neq \rho_{2}$.

Os dados epelip1.txt são os correspondentes ao número diário de ataques epilépticos de um paciente durante 204 dias.

A seguir apresentamos a notação usada ao longo deste programa.

$$
\begin{array}{ll}
I 1=1\{m=0\} & I 2=1\{m=N\} \quad \text { comb }=\left(\begin{array}{l}
N \\
m
\end{array}\right) \\
\text { factm }=m ! & A 1=\left(\alpha_{2}-\alpha_{2} p+\alpha_{1} p\right)^{m}\left(1-\alpha_{2}+\alpha_{2} p-\alpha_{1} p\right)^{N-m} \\
\text { factN }=N ! & A 2=\left(\alpha_{2}-\alpha_{2} p+\alpha_{1} p\right)^{m-1}\left(1-\alpha_{2}+\alpha_{2} p-\alpha_{1} p\right)^{N-m-1} \\
\text { factm } 1=(N-m) ! & A 3=\left(\alpha_{2}-\alpha_{2} p+\alpha_{1} p\right)^{m-2}\left(1-\alpha_{2}+\alpha_{2} p-\alpha_{1} p\right)^{N-m-2} \\
P 1=(1-p)^{m} p^{N-m} & R 1=m-N \alpha_{1} p \\
P 2=(1-p)^{m-1} p^{N-m-1} & R 2=m-N\left(1-\alpha_{1}+\alpha_{1} p\right) \\
P 3=(1-p)^{m-2} p^{N-m-2} & R 3=m-N\left(\alpha_{2}-\alpha_{2} p+\alpha_{1} p\right) \\
Q 1=p^{m}(1-p)^{N-m} & R 4=m-N\left(\alpha_{2}-\alpha_{2} p\right) \\
Q 2=p^{m-1}(1-p)^{N-m-1} & R 5=m-N\left(\alpha_{2}+p-\alpha_{2} p\right)
\end{array}
$$




$$
\begin{aligned}
& Q 3=p^{m-2}(1-p)^{N-m-1} \\
& R 6=m-N p \\
& B 1=\left(\alpha_{2}-\alpha_{2} p\right)^{m}\left(1-\alpha_{2}+\alpha_{2} p\right)^{N-m} \\
& R 7=m-N(1-p) \\
& B 2=\left(\alpha_{2}-\alpha_{2} p\right)^{m-1}\left(1-\alpha_{2}+\alpha_{2} p\right)^{N-m-1} \\
& R 8=\left(1-\alpha_{1} p\right)^{2} \\
& B 3=\left(\alpha_{2}-\alpha_{2} p\right)^{m-2}\left(1-\alpha_{2}+\alpha_{2} p\right)^{N-m-2} \\
& R 9=\left(\alpha_{1} p\right)^{2} \\
& C 1=\left(\alpha_{1} p\right)^{m}\left(1-\alpha_{1} p\right)^{N-m} \\
& R 10=\left(\alpha_{1}-\alpha_{1} p\right)^{2} \\
& C 2=\left(\alpha_{1} p\right)^{m-1}\left(1-\alpha_{1} p\right)^{N-m-1} \\
& C 3=\left(\alpha_{1} p\right)^{m-2}\left(1-\alpha_{1} p\right)^{N-m-2} \\
& D 1=\left(\alpha_{2}+p-\alpha_{2} p\right)^{m}\left(1-\alpha_{2}-p+\alpha_{2} p\right)^{N-m} \\
& R 11=\left(1-\alpha_{1}+\alpha_{1} p\right)^{2} \\
& R 12=\left(1-\alpha_{2}+\alpha_{2} p-\alpha_{1} p\right)^{2} \\
& D 2=\left(\alpha_{2}+p-\alpha_{2} p\right)^{m-1}\left(1-\alpha_{2}-p+\alpha_{2} p\right)^{N-m-1} \\
& R 13=\left(\alpha_{2}-\alpha_{2} p+\alpha_{1} p\right)^{2} \\
& D 3=\left(\alpha_{2}+p-\alpha_{2} p\right)^{m-2}\left(1-\alpha_{2}-p+\alpha_{2} p\right)^{N-m-2} \\
& R 14=\left(1-\alpha_{2}+\alpha_{2} p\right)^{2} \\
& E 1=\left(1-\alpha_{1}+\alpha_{1} p\right)^{m}\left(\alpha_{1}-\alpha_{1} p\right)^{N-m} \\
& R 15=\left(\alpha_{2}-\alpha_{2} p\right)^{2} \\
& E 2=\left(1-\alpha_{1}+\alpha_{1} p\right)^{m-1}\left(\alpha_{1}-\alpha_{1} p\right)^{N-m-1} \\
& E 3=\left(1-\alpha_{1}+\alpha_{1} p\right)^{m-2}\left(\alpha_{1}-\alpha_{1} p\right)^{N-m-2} \\
& R 16=\left(1-\alpha_{2}-p+\alpha_{2} p\right)^{2} \\
& R 17=\left(\alpha_{2}+p-\alpha_{2} p\right)^{2} \\
& R 19=\alpha_{1}-\alpha_{2} \\
& \mathrm{drhol}=\frac{\partial f}{\partial \rho_{1}} \\
& \operatorname{drho} 2=\frac{\partial f}{\partial \rho_{2}} \\
& \text { dalpha1 }=\frac{\partial f}{\partial \alpha_{1}} \\
& \text { dalpha2 }=\frac{\partial f}{\partial \alpha_{2}} \\
& \mathrm{dp}=\frac{\partial f}{\partial p_{1}} \\
& \mathrm{~d} 2 \mathrm{rhol}=\frac{\partial^{2} f}{\partial \rho_{1}^{2}} \\
& \mathrm{~d} 2 \mathrm{rho} 2=\frac{\partial^{2} f}{\partial \rho_{2}^{2}} \\
& \text { d2alpha1 }=\frac{\partial^{2} f}{\partial \alpha_{1}^{2}} \\
& \text { d2alpha2 }=\frac{\partial^{2} f}{\partial \alpha_{2}^{2}} \\
& \mathrm{~d} 2 \mathrm{p}=\frac{\partial^{2} f}{\partial p_{1}^{2}} \\
& R 18=\left(\alpha_{1}-\alpha_{1} p\right) \\
& \text { d2rholrho2 }=\frac{\partial^{2} f}{\partial \rho_{1} \partial \rho_{2}} \\
& \text { d2rholalpha1 }=\frac{\partial^{2} f}{\partial \rho_{1} \partial \alpha_{1}} \\
& \text { d2rholalpha2 }=\frac{\partial^{2} f}{\partial \rho_{1} \partial \alpha_{2}} \\
& \text { d2rholp }=\frac{\partial^{2} f}{\partial \rho_{1} \partial p_{1}} \\
& \text { d2rho2alpha1 }=\frac{\partial^{2} f}{\partial \rho_{2} \partial \alpha_{1}} \\
& \text { d2rho2alpha2 }=\frac{\partial^{2} f}{\partial \rho_{2} \partial \alpha_{2}} \\
& \mathrm{~d} 2 \mathrm{rho} 2 \mathrm{p}=\frac{\partial^{2} f}{\partial \rho_{2} \partial p_{1}} \\
& \text { d2alpha1alpha2 }=\frac{\partial^{2} f}{\partial \alpha_{1} \partial \alpha_{2}} \\
& \text { d2alpha1p }=\frac{\partial^{2} f}{\partial \alpha_{1} \partial p_{1}} \\
& \text { d2alpha2p }=\frac{\partial^{2} f}{\partial \alpha_{2} \partial p_{1}}
\end{aligned}
$$




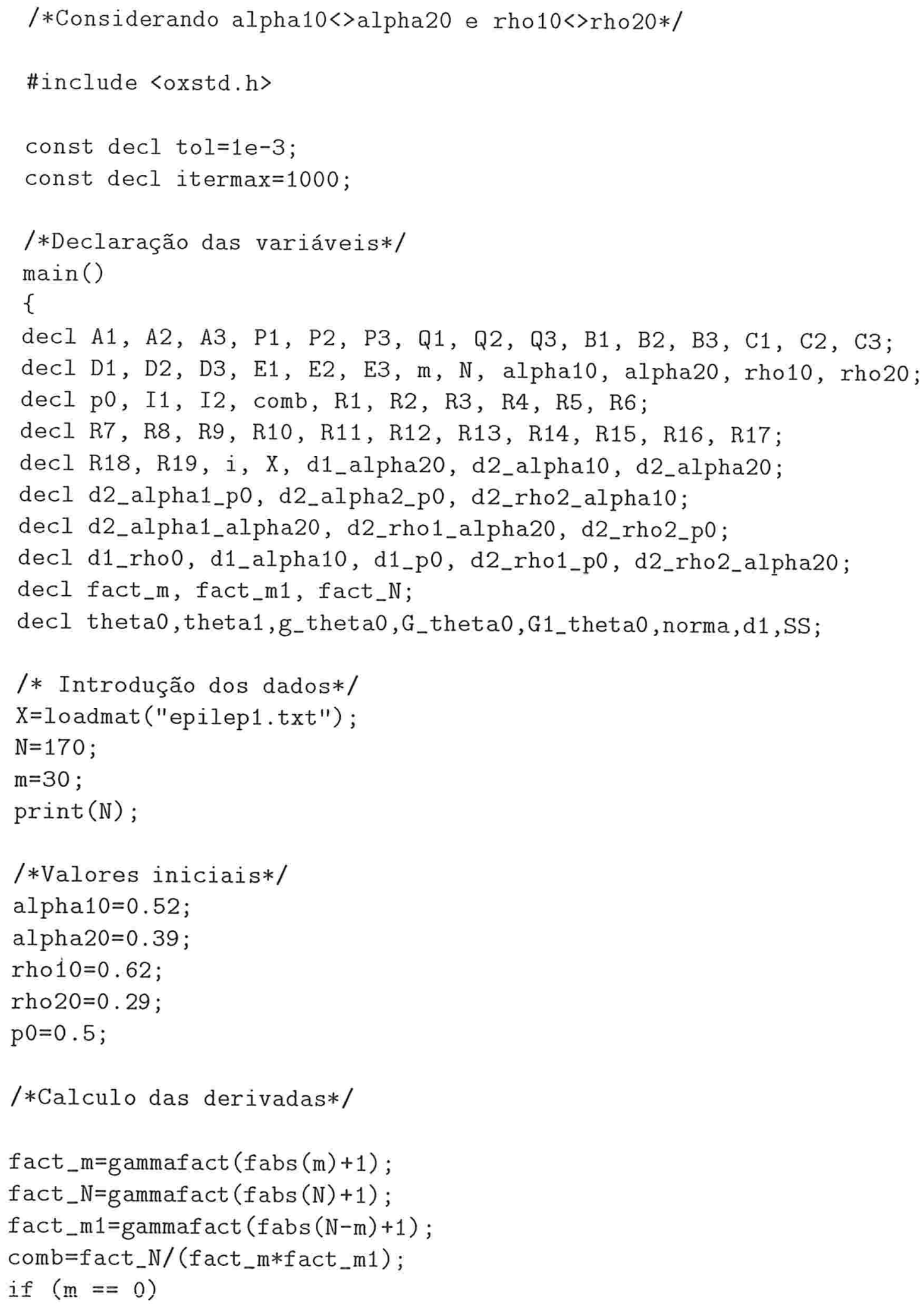




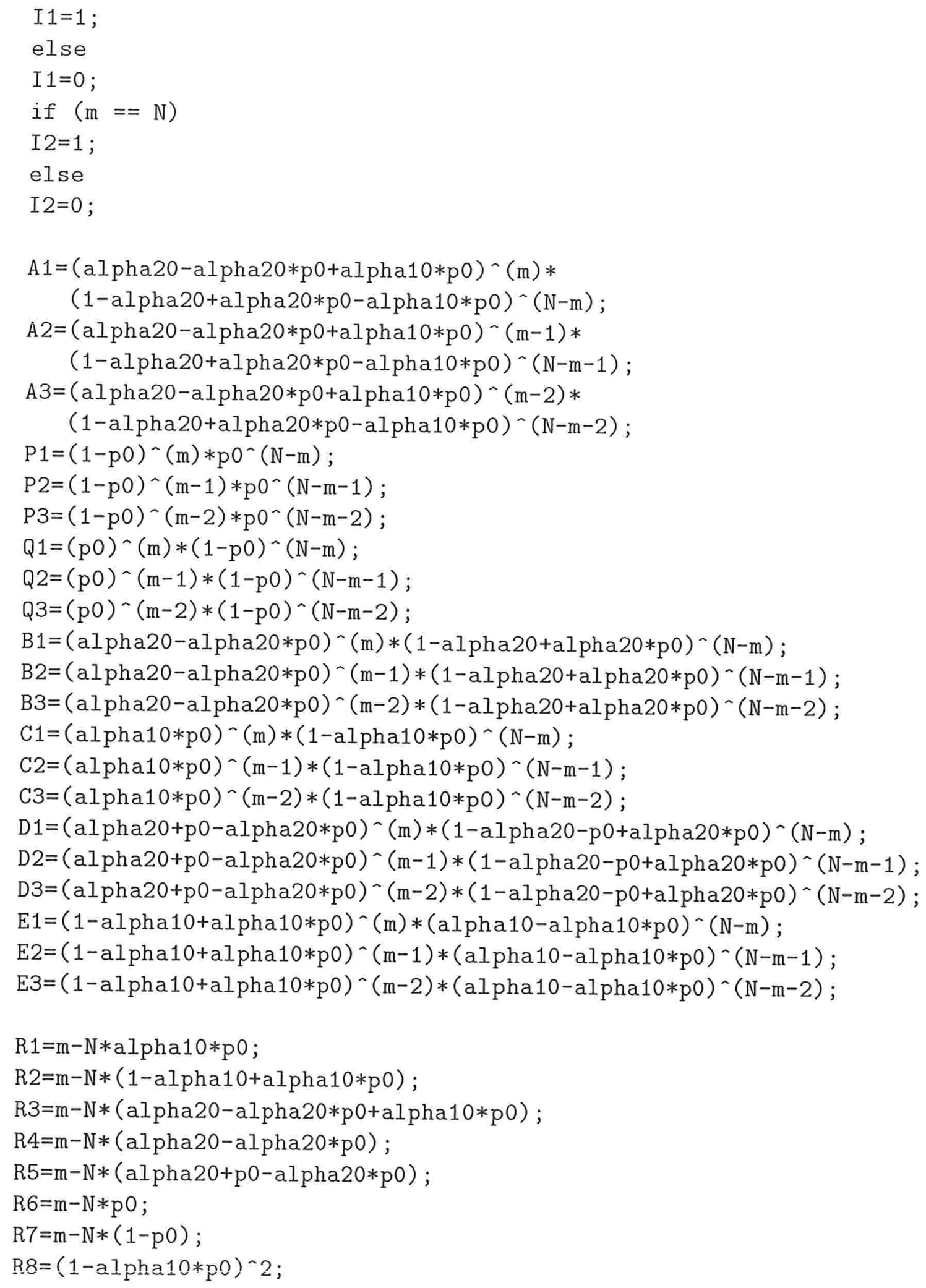




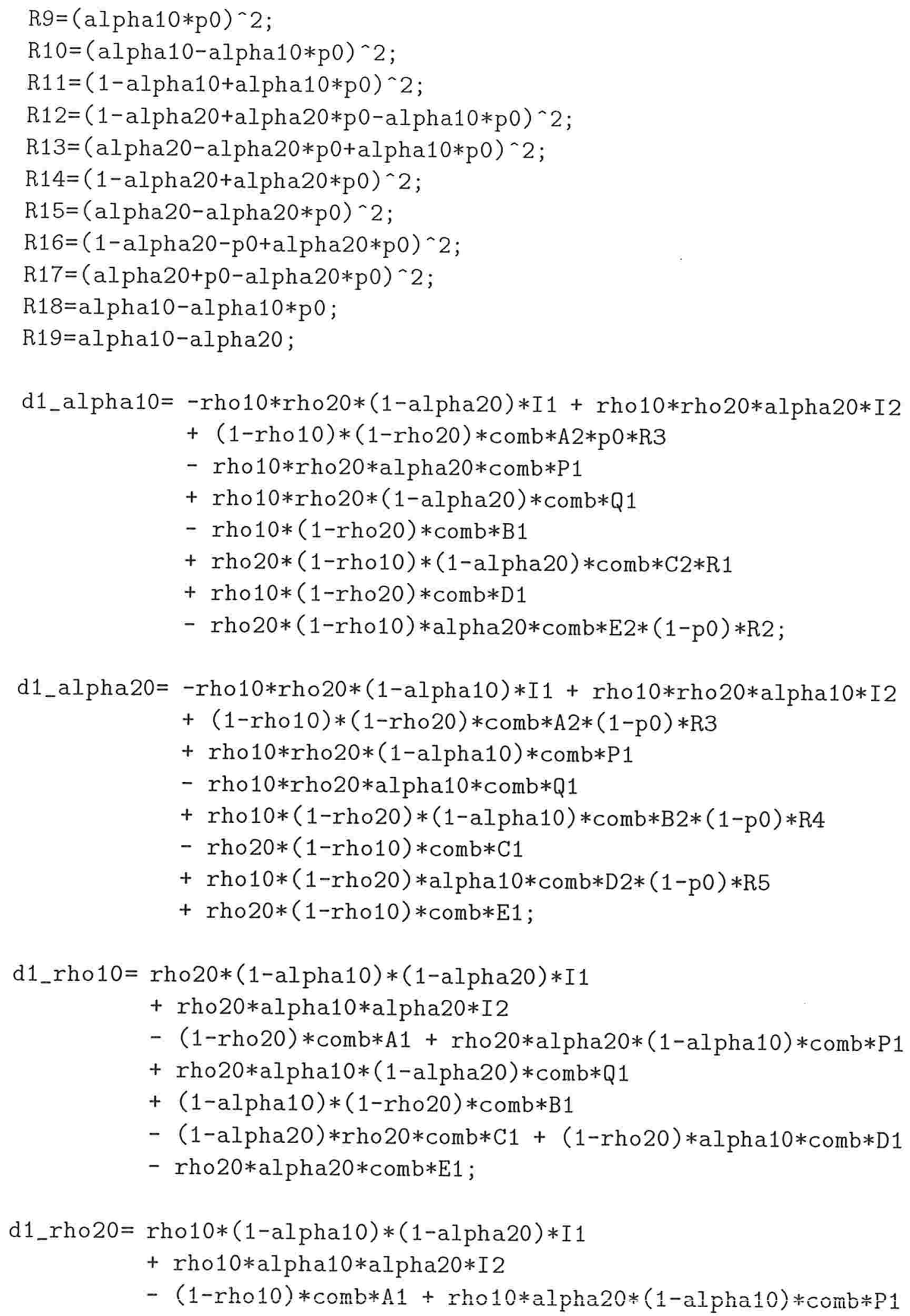




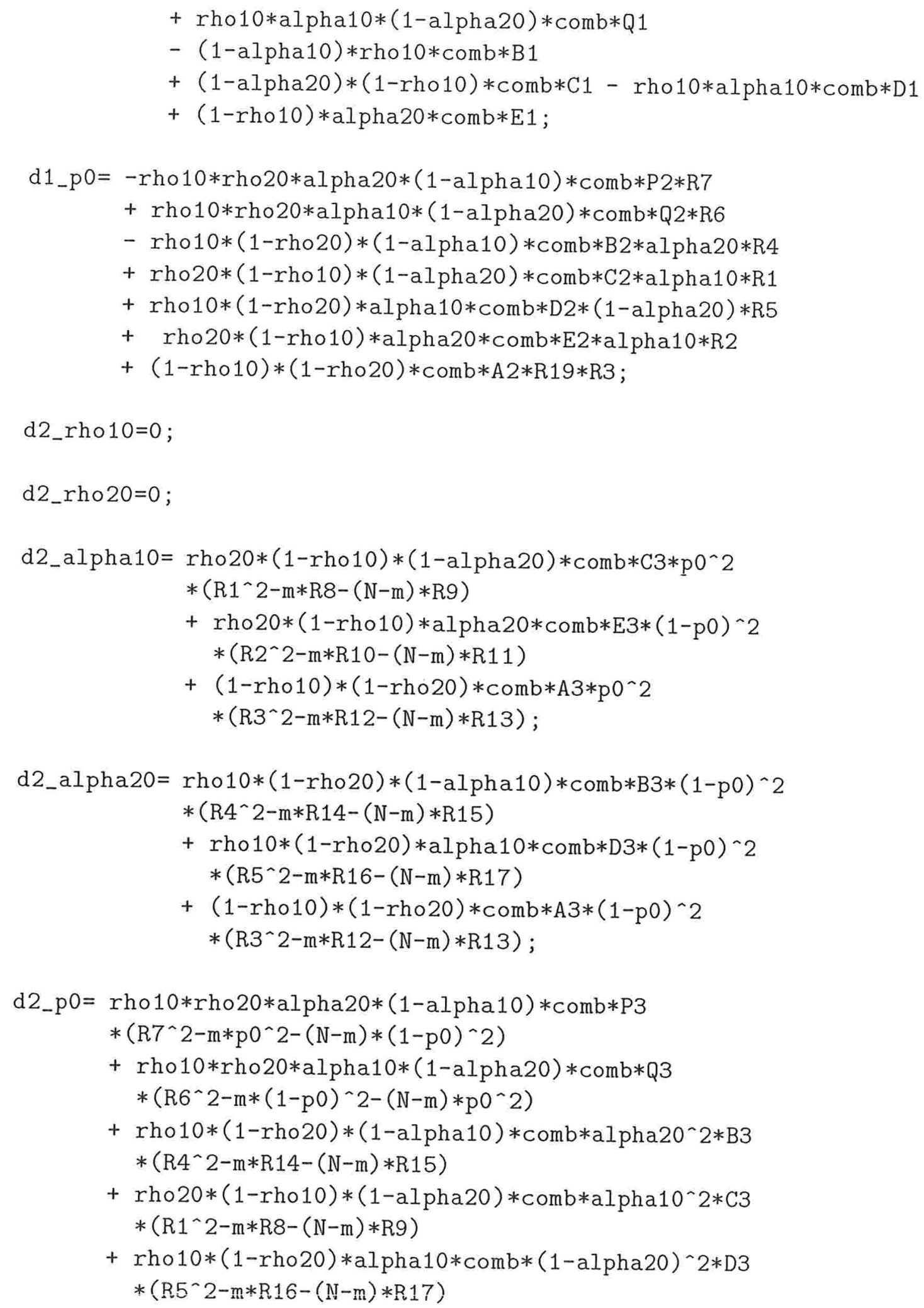


+ rho20*(1-rho10)*alpha20*comb*alpha10^2*E3

$*\left(\mathrm{R} 2{ }^{\wedge} 2-\mathrm{m} * \mathrm{R} 10-(\mathrm{N}-\mathrm{m}) * \mathrm{R} 11\right)$

$+(1-r h o 10) *(1-r h o 20) * \operatorname{comb} * A 3 * R 19^{\wedge} 2 *\left(R^{\wedge} 2-m * R 12-(N-m) * R 13\right)$;

d2_rho1_rho20 $=(1-$ alpha10 $) *(1-$ alpha20 $) * I 1+$ alpha10*alpha $20 * I 2$

+ alpha20*(1-alpha10)*comb*P1

+ alpha10*alpha20*comb*Q1

- (1-alpha10)*comb*B1 - (1-alpha20)*comb*C1

- alpha10*comb*D1 - alpha20*comb*E1 + comb*A1;

d2_rho1_alpha10 $=-\operatorname{rho} 20 *(1-$ alpha20 $) * I 1+$ rho $20 * a l p h a 20 * I 2$

- rho20*alpha20*comb*P1

+ rho20*(1-alpha20)*comb*Q1

- $(1-\mathrm{rho} 20) *$ comb*B1

- rho20*(1-alpha20)*comb*C2*p0*R1

$+(1-r h \circ 20) *$ comb $* \mathrm{D} 1$

+ rho20*alpha $20 *$ comb*E $1 *(1-\mathrm{p} 0) * \mathrm{R} 2$

- $(1-r h o 20) *$ comb*A2*p0*R3;

d2_rho1_alpha20 $=-\operatorname{rho} 20 *(1-\mathrm{alpha10}) * \mathrm{I} 1+$ rho20*alpha10*I2

+ rho $20 *(1-a l$ pha10 $) *$ comb*P1

- rho20*alpha10*comb*Q1

$+(1-r h o 20) *(1-a l p h a 10) *$ comb $* B 2 *(1-p 0) * \mathrm{R} 4$

$+\mathrm{rho} 20 * \mathrm{comb} * \mathrm{C} 1$

$+(1-r h o 20) * a l p h a 10 *$ comb $* \mathrm{D} 2 *(1-\mathrm{p} 0) * \mathrm{R} 5$

- rho20*comb*E1 - (1-rho20)*comb*A2*(1-p0)*R3;

d2_rho1_p0 $=-$ rho20*alpha20*(1-alpha10)*comb*P $2 * \mathrm{R} 7$

+ rho20*alpha10*(1-alpha20)*comb*Q1*R6

- (1-rho20)*(1-alpha10)*comb*alpha $20 * \mathrm{~B} 2 * \mathrm{R} 4$

- rho $20 *(1-$ alpha20)*comb*C2*R1

$+(1-r h o 20) * a l$ pha $10 *$ comb $* \mathrm{D} 2 *(1-\mathrm{al}$ pha 20$) * \mathrm{R} 5$

+ rho20*alpha20*alpha10*comb*E $2 * \mathrm{R} 2$

- (1-rho20)*comb*A2*R19*R3;

d2_rho2_alpha10 $=-\operatorname{rho10} *(1-\mathrm{alpha} 20) * \mathrm{I} 1+\operatorname{rho} 10 * a l$ pha $20 * \mathrm{I} 2$

- rho10*alpha20*comb*P1

+ rho10*(1-alpha20)*comb*Q1

+ rho10*comb*(B1-D1)

$+(1-r h o 10) *(1-r h o 10) * \operatorname{comb} * \mathrm{C} 2 * \mathrm{p} 0 * \mathrm{R} 1$

- (1-rho10)*alpha20*comb*D2*(1-p0)*R2 
- $(1-r h o 10) *$ comb*A2*p0*R3;

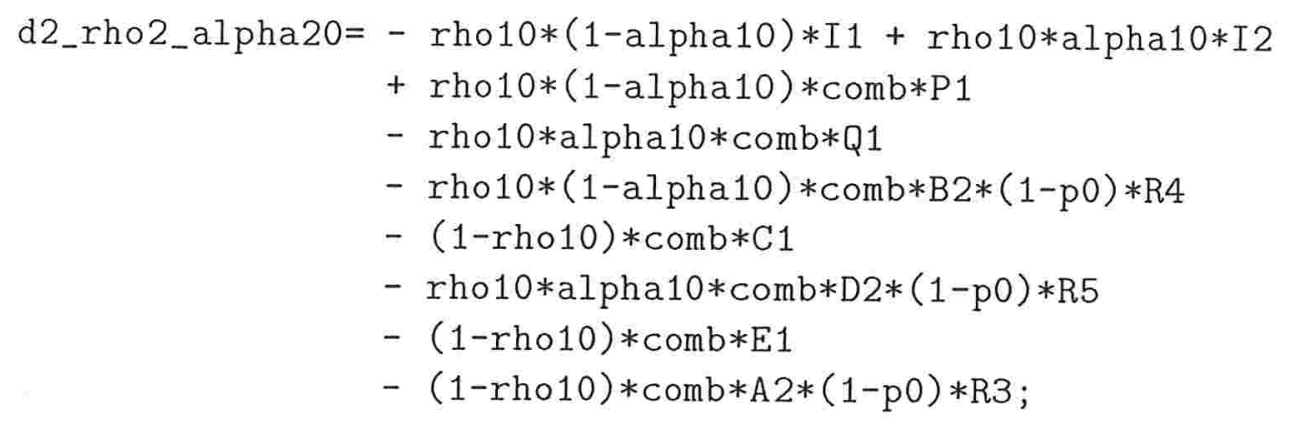

d2_rho2_p0 $=-$ rho10*alpha20*(1-alpha10)*comb*P2*R7

+ rho10*alpha10*(1-alpha20)*comb*Q1*R6

+ rho10*(1-alpha10)*comb*alpha20*B2*R4

$+(1-r h o 10) *(1-a l$ pha 20$) *$ comb $* \mathrm{C} 2 * \mathrm{R} 1$

- rho $10 * a l$ pha $10 *$ comb*D $2 *(1-$ alpha 20$) * \mathrm{R} 5$

$+(1-r h o 10) * a l p h a 20 * a l$ pha $10 * \operatorname{comb} * \mathrm{E} 2 * \mathrm{R} 2$

- $(1-r h \circ 10) *$ comb*A2*R19*R3;

d2_alpha1_alpha20 $=$ rho10*rho20*(I1+I2) - rho10*rho20*comb*(P1+Q1)

- rho10*(1-rho20)*comb*B2*(1-p0)*R4

- rho $20 *(1-$ rho 10$) *$ comb*C $2 *$ p $0 * \mathrm{R} 1$

$+\mathrm{rho} 10 *(1-\mathrm{rho} 20) * \mathrm{comb} * \mathrm{D} 2 *(1-\mathrm{p} 0) * \mathrm{R} 5$

- rho20*(1-rho10)*comb*E2*(1-p0)*R1

$+(1-r h o 10) *(1-r h o 20) *$ comb $* A 3 *$ p0 $*(1-p 0)$ *(R3^2-m*R12-(N-m)*R13);

d2_alpha1_p0 $=$ rho $10 *$ rho $20 * a l$ pha $20 *$ comb $* \mathrm{P} 2 * \mathrm{R} 7$

+ rho10*rho $20 *(1-a l$ pha 20$) *$ comb*Q $2 * R 6$

+ rho10*(1-rho20)*comb*B2*alpha20*R4

+ rho $20 *(1-$ rho 10$) *(1-\mathrm{alpha} 20) * \operatorname{comb} * \mathrm{C} 3 *($ alpha10*p0) $*(\mathrm{R} 1 \sim 2-(\mathrm{N}-\mathrm{m}) *($ alpha10*p0) $)$

+ rho20*(1-rho10)*alpha20*comb*E3*R18*(R2^2-m*R18)

$+(1-r h o 10) *(1-r h o 20) *$ comb*A2*R3

$+(1-r h o 10) *(1-r h o 20) *$ comb $* A 3 * \mathrm{R} 19 * \mathrm{p} 0$ *(R3^2-m*R12-(N-m)*R13);

d2_alpha2_p0 $=-(1-r h o 10) *(1-r h o 20) * \operatorname{comb} * A 2 * \mathrm{R} 3$

- rho10*rho20*(1-alpha10)*comb*P2*R7

- rho10*rho $20 * a l$ pha $10 *$ comb*Q $2 * R 6$

- rho10*(1-rho20)*(1-alpha10)*comb*B2*R4 


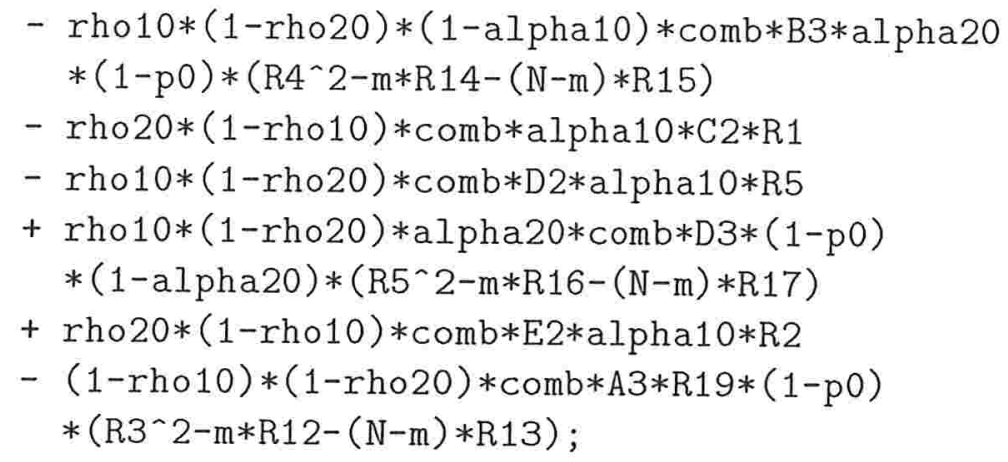

/*Vetor de parametros*/

theta $0=a l p h a 10 \mid$ alpha20 $\mid$ rho10 $\mid$ rho20 $\mid$ p0;

print ("theta $0="$, theta 0$)$;

/*Vetor de primeiras derivadas*/

g_theta0=d1_alpha10 $\mid d 1$ _alpha20 $\mid$ d1_rho10|d1_rho20|d1_p0;

/*Matriz Hessiana*/

G_theta0 $=$ d2_alpha10 d2_alpha1_alpha20 d2_rho1_alpha10

“d2_rho2_alpha10 d2_alpha1_p0l

d2_alpha1_alpha20 〜2_alpha20 d2_rho1_alpha20

〜2_rho2_alpha20 d2_alpha2_p01

d2_rho1_alpha10 d2_rho1_alpha20 d2_rho10

nd2_rho1_rho20 d2_rho1_pol

d2_rho2_alpha10 d2_rho2_alpha20 d2_rho1_rho20

n2_rho20 d2_rho2_p01

d2_alpha1_p0 ${ }^{\sim}$ 2_alpha2_p0 d2_rho1_p0

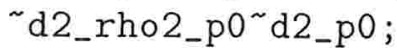

G1_theta0 $=$ invert $\left(G_{-}\right.$theta 0$)$;

/*Calculo do algoritmo de Newton-Raphson*/

norma $=1$;

$\mathrm{d} 1=0$;

while (norma $>$ tol \&\& $d 1<$ itermax) \{

theta1=theta0 - G1_theta0*g_theta0; 


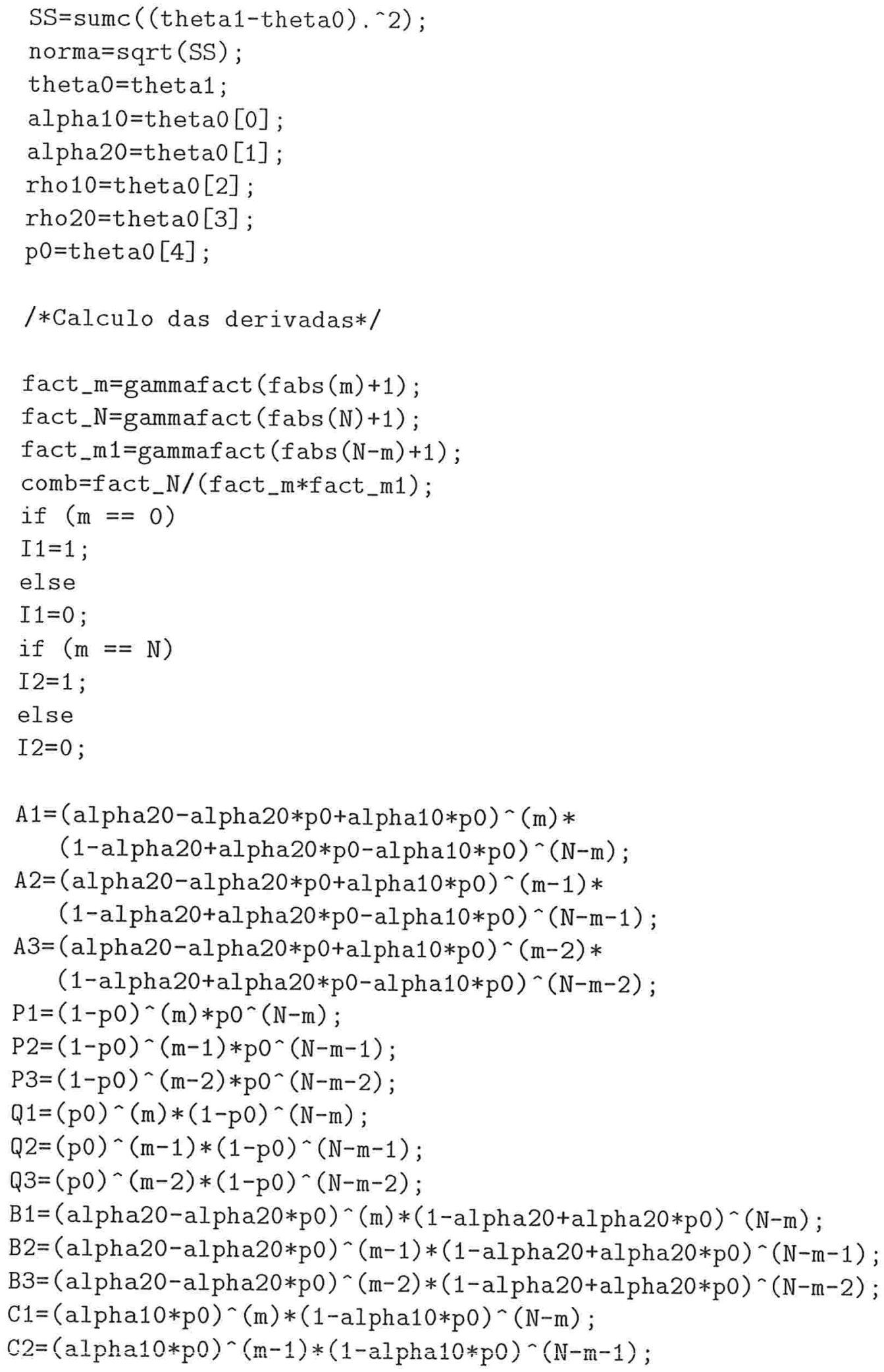




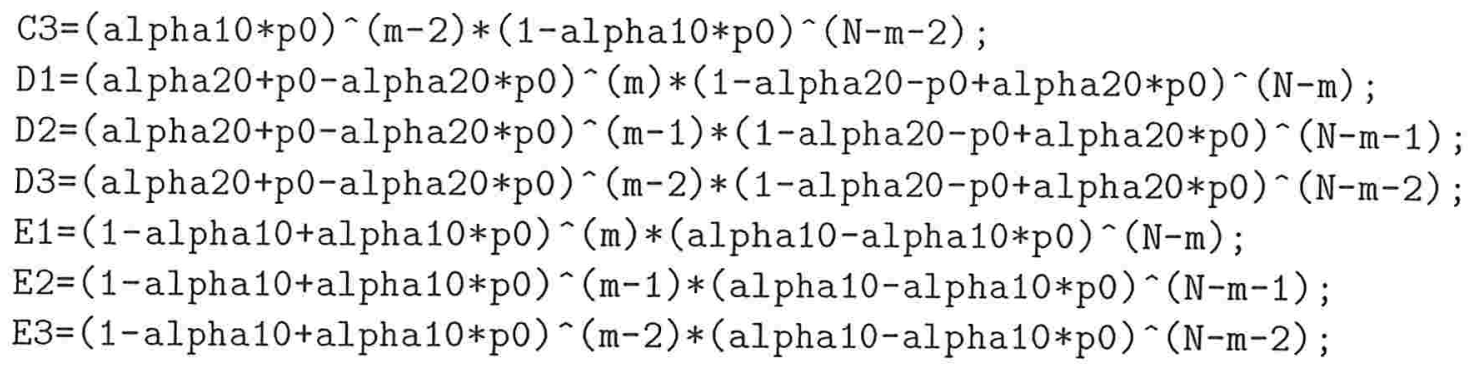


+ rho10*(1-rho20)*(1-alpha10)*comb*B2*(1-p0)*R4

- rho $20 *(1-r h o 10) *$ comb*C1

+ rho10*(1-rho20)*alpha10*comb*D2*(1-p0)*R5

+ rho $20 *(1-r h o 10) *$ comb*E1;

d1_rho10 $=$ rho $20 *(1-$ alpha 10$) *(1-$ alpha20 $) * I 1$

+ rho20*alpha10*alpha20*I2

- (1-rho20)*comb*A1 + rho20*alpha $20 *(1-a l$ pha10)*comb*P1

+ rho20*alpha10*(1-alpha20)*comb*Q1

$+(1-\mathrm{alpha} 10) *(1-\mathrm{rho} 20) *$ comb $* \mathrm{~B} 1$

- (1-alpha20)*rho20*comb*C1 + (1-rho20)*alpha10*comb*D1

- rho $20 *$ alpha $20 *$ comb*E1;

d1_rho20 $=$ rho $10 *(1-\mathrm{alpha} 10) *(1-\mathrm{alpha} 20) * \mathrm{I} 1$

+ rho10*alpha10*alpha20*I2

- (1-rho10)*comb*A1 + rho10*alpha20*(1-alpha10)*comb*P1

+ rho10*alpha10*(1-alpha20)*comb*Q1

- (1-alpha10)*rho10*comb*B1

$+(1-a l p h a 20) *(1-r h o 10) *$ comb*C1 - rho10*alpha10*comb*D1

$+(1-r h \circ 10) * a l$ pha20*comb*E1;

d1_p0 $=-r h o 10 * r h o 20 * a l$ pha20*(1-alpha10 $) *$ comb $* \mathrm{P} 2 * \mathrm{R} 7$

+ rho10*rho $20 * a l$ pha10*(1-alpha20)*comb*Q2*R6

- rho10*(1-rho20)*(1-alpha10)*comb*B2*alpha20*R4

+ rho $20 *(1-r h o 10) *(1-a l$ pha 20$) *$ comb $* C 2 * a l$ pha $10 * \mathrm{R} 1$

+ rho10*(1-rho20)*alpha10*comb*D2*(1-alpha20)*R5

+ rho20*(1-rho10)*alpha20*comb*E $2 * a l$ pha10*R 2

$+(1-r h o 10) *(1-r h o 20) *$ comb $* A 2 * R 19 * \mathrm{R} 3$;

d2_rho10=0;

d2_rho20=0;

d2_alpha10 $=r h o 20 *(1-r h o 10) *(1-$ alpha20 $) *$ comb $*$ C $3 *$ p0^2

$*(\mathrm{R} 1 \sim 2-\mathrm{m} * \mathrm{R} 8-(\mathrm{N}-\mathrm{m}) * \mathrm{R} 9)$

+ rho $20 *(1-r h o 10) * a l p h a 20 * \operatorname{comb} * \mathrm{E} 3 *(1-\mathrm{p} 0)^{\wedge} 2$ $*(\mathrm{R} 2 \wedge 2-\mathrm{m} * \mathrm{R} 10-(\mathrm{N}-\mathrm{m}) * \mathrm{R} 11)$

$+(1-r h o 10) *(1-r h o 20) *$ comb $* \mathrm{~A}_{3} * \mathrm{p}^{\wedge} 2$

$*\left(\mathrm{R} 3^{\sim} 2-\mathrm{m} * \mathrm{R} 12-(\mathrm{N}-\mathrm{m}) * \mathrm{R} 13\right)$;

d2_alpha20 $=r h o 10 *(1-r h o 20) *(1-$ alpha 10$) * c o m b * B 3 *(1-p 0)^{\wedge} 2$ 


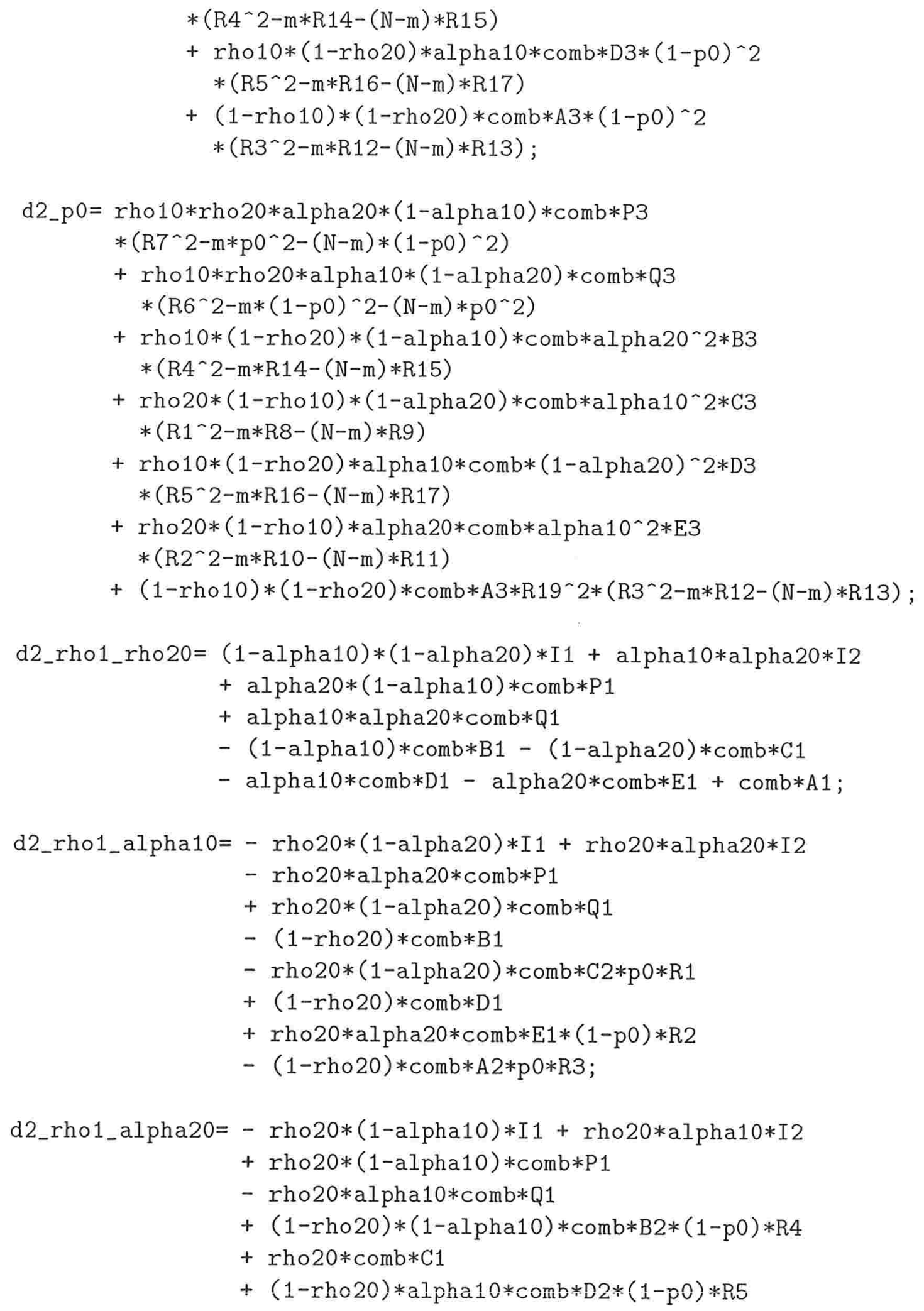


- rho $20 *$ comb*E1 - (1-rho20)*comb*A2*(1-p0)*R3;

d2_rho1_p0 $=-$ rho $20 * a l$ pha2 $2 *(1-$ alpha10 $) *$ comb $* \mathrm{P} 2 * \mathrm{R} 7$

+ rho20*alpha10*(1-alpha20)*comb*Q1*R6

- $(1-r h o 20) *(1-a l$ pha10 $) *$ comb*al pha $20 * \mathrm{~B} 2 * \mathrm{R} 4$

- rho20*(1-alpha20)*comb*C2*R1

$+(1-r h o 20) * a l p h a 10 *$ comb $*$ D $2 *(1-a l p h a 20) * \mathrm{R} 5$

+ rho $20 * a l$ pha $20 * a l$ pha10*comb*E2*R2

- (1-rho20)*comb*A2*R19*R3;

d2_rho2_alpha10 $=-\operatorname{rho10} *(1-\mathrm{al}$ pha20 $) * I 1+$ rho10*alpha20*I2

- rho10*alpha $20 *$ comb*P1

+ rho10*(1-alpha20)*comb*Q1

+ rho10*comb*(B1-D1)

$+(1-r h o 10) *(1-r h \circ 10) * \operatorname{comb} * \mathrm{C} 2 * \mathrm{p} 0 * \mathrm{R} 1$

- $(1-\mathrm{rho} 10) * \mathrm{alpha} 20 * \mathrm{comb} * \mathrm{D} 2 *(1-\mathrm{p} 0) * \mathrm{R} 2$

- $(1-r h o 10) *$ comb*A2*p0*R3;

d2_rho2_alpha20 $=-\operatorname{rho10} *(1-\mathrm{alpha10}) * \mathrm{I} 1+$ rho10*alpha10*I2

+ rho10*(1-alpha10)*comb*P1

- rho10*alpha10*comb*Q1

- $\operatorname{rho10}(1-\mathrm{alpha10}) * \operatorname{comb} * \mathrm{~B} 2 *(1-\mathrm{p} 0) * \mathrm{R} 4$

- (1-rho10)*comb*C1

- rho10*alpha10*comb*D2*(1-p0)*R5

- $(1-\mathrm{rho} 10) * \mathrm{comb} * \mathrm{E} 1$

- $(1-r h o 10) *$ comb*A2*(1-p0)*R3;

d2_rho2_p0 $=-$ rho10*alpha20*(1-alpha10)*comb*P2*R7

+ rho10*alpha10*(1-alpha20)*comb*Q1*R6

+ rho10*(1-alpha10)*comb*alpha20*B2*R4

$+(1-r h o 10) *(1-$ alpha 20$) * \operatorname{comb} * \mathrm{C} 2 * \mathrm{R} 1$

- rho10*alpha10*comb*D $2 *(1-$ alpha 20$) * \mathrm{R} 5$

$+(1-r h o 10) * a l p h a 20 * a l$ pha10*comb*E2 $* \mathrm{R} 2$

- (1-rho10)*comb*A2*R19*R3;

d2_alpha1_alpha20 $=$ rho $10 *$ rho $20 *(I 1+I 2)-\operatorname{rho} 10 * r h o 20 * \operatorname{comb} *(\mathrm{P} 1+\mathrm{Q} 1)$

- rho10*(1-rho20)*comb*B2*(1-p0)*R4

- rho20*(1-rho10)*comb*C2*p0*R1

$+\mathrm{rho} 10 *(1-\mathrm{rho} 20) * \mathrm{comb} * \mathrm{D} 2 *(1-\mathrm{p} 0) * \mathrm{R} 5$

- rho $20 *(1-\mathrm{rho} 10) *$ comb $* \mathrm{E} 2 *(1-\mathrm{p} 0) * \mathrm{R} 1$

$+(1-r h o 10) *(1-r h o 20) * \operatorname{comb} * \Lambda 3 * \mathrm{p} 0 *(1-\mathrm{p} 0)$ 


$$
*\left(\mathrm{R}^{\sim} 2-\mathrm{m} * \mathrm{R} 12-(\mathrm{N}-\mathrm{m}) * \mathrm{R} 13\right) \text {; }
$$

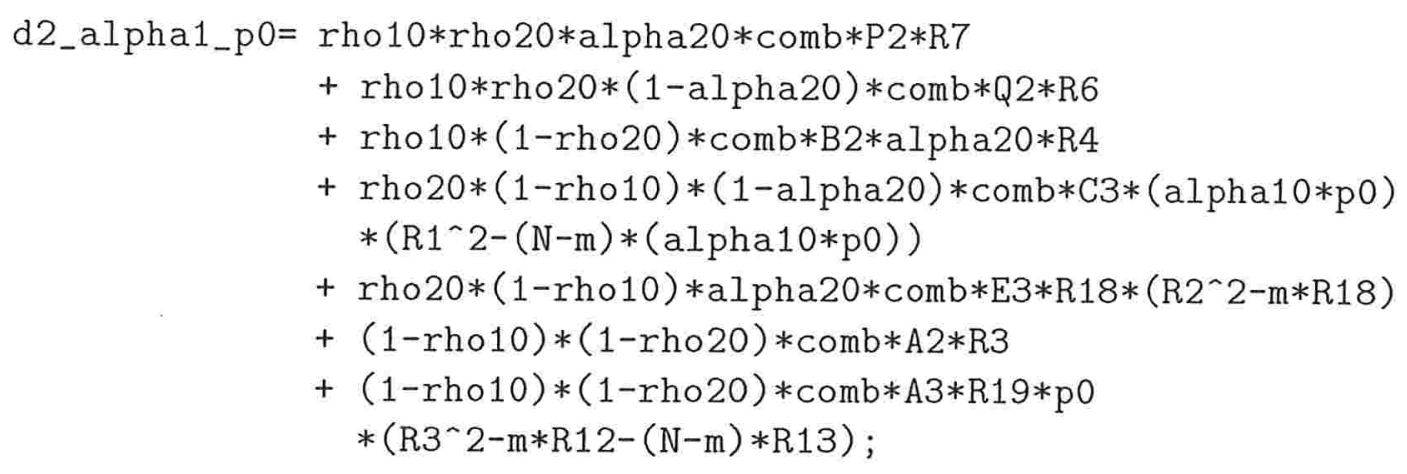

d2_alpha2_p0 $=-(1-r h o 10) *(1-r h o 20) * \operatorname{comb} * A 2 * R 3$

- rho10*rho $20 *(1-$ alpha10 $) *$ comb $* P 2 * R 7$

- rho10*rho $20 * a l$ pha10*comb*Q2*R6

- rho10*(1-rho20)*(1-alpha10)*comb*B2*R4

- rho10*(1-rho20)*(1-alpha10)*comb*B3*alpha20 $*(1-\mathrm{p} 0) *\left(\mathrm{R} 4^{\wedge} 2-\mathrm{m} * \mathrm{R} 14-(\mathrm{N}-\mathrm{m}) * \mathrm{R} 15\right)$

- rho $20 *(1-r h o 10) *$ comb*alpha10*C2*R1

- rho10*(1-rho20)*comb*D2*alpha10*R5

+ rho10*(1-rho20)*alpha20*comb*D3*(1-p0) $*(1-\mathrm{alpha} 20) *\left(\mathrm{R} 5^{\wedge} 2-\mathrm{m} * \mathrm{R} 16-(\mathrm{N}-\mathrm{m}) * \mathrm{R} 17\right)$

$+\mathrm{rho} 20 *(1-\mathrm{rho} 10) *$ comb $* \mathrm{E} 2 *$ alpha10*R2

- $(1-r h o 10) *(1-r h o 20) *$ comb*A3*R19*(1-p0) *(R3^2-m*R12-(N-m)*R13);

I*Vetor de parametros*/

theta $0=a l$ pha10 $\mid$ alpha20 $\mid$ rho10 $\mid$ rho20 $\mid$ p0;

print ("theta0 $=$ ", theta0);

/*Vetor de primeiras derivadas*/

g_theta0 =d1_alpha10 $\mid d 1$ _alpha20 $\mid$ d1_rho10 $\mid$ d1_rho20 $\mid$ d1_p0;

/*Matriz Hessiana*/

G_theta0 $=$ d2_alpha10 d2_alpha1_alpha20 d2_rho1_alpha10

rd2_rho2_alpha10 d2_alpha1_p0l

d2_alpha1_alpha20 d2_alpha20 d2_rho1_alpha20 


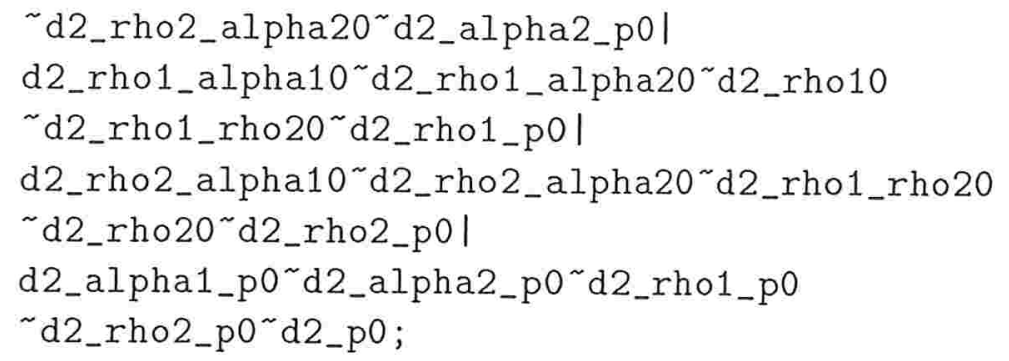




\section{Referências Bibliográficas}

[1] Alberts, W. (1999). Stop-loss premiums under dependence. Insurance: Mathematics and Economics 24, 173-185.

[2] Al-Osh, M.A. and Alzaid, A.A. (1987). First-order integer-valued autoregressive (INAR(1)) process, Journal of Time Series Analysis 8, 261-275.

[3] Ambagastipitiya, R.S., (1998). On the distribution of a sum of correlated aggregated claims. Insurance: Mathematics and Economics 23, 15-19.

[4] Ambagaspitiya, R.S. (1999). On the distribution of two classes of correlated aggregate claims. Insurance: Mathematics and Economics 24, 301-308.

[5] Asmussen, S., (1989). Risk theory in a Markovian environment. Scandinavian Actuarial Journal 23, 69-100.

[6] Blishke, W. R. (1962). Moment estimators for the parameters of a mixture of two binomial distributions. Annals of Mathematical Statistics 33, 444-454.

[7] Bowers, N., Gerber, H., Hickman, J., Jones, D. and Nesbitt, C. (1997). Actuarial Mathematics. Schaumburg, Illinois.

[8] Daykin, C.D., Pentikäinen, T. and Pesonen, M. (1994). Practical Risk Theory for Actuaries. London: Chapman and Hall.

[9] DeFinetti, B. (1975). Theory of Probability. Vol 2. New York: Wiley. 
[10] Dhaene, J. and Gooverts, M. (1996). Dependency of risks and stop-loss order. In: ASTIN Bulletin, 26, 201-212.

[11] Dhaene, J. and Gooverts, M. (1997). On the dependency of risks in the individual life model. Insurance: Mathematics and Economics, 19, 243-253.

[12] Dhaene, J. and Denuit, M. (1999). The safest dependence structure among risk. Insurance: Mathematics and Economics 25, 11-21.

[13] Dhaene, J., Wang, S., Young, V. and Goovaerts, M. (2000). Comonotonicity and maximal stop-loss premiums. Bulletin of the Swiss Association of Actuaries 2, 99-113.

[14] Dhaene, J., Denuit, M., Goovaerts, M.J., Kaas, R. and Vyncke, D. (2002a). The concept of comonotonicity in actuarial science and finance: theory. Insurance: Mathematics and Economics 31, 133-161.

[15] Dhaene, J., Denuit, M., Goovaerts, M.J., Kaas, R. and Vyncke, D. (2002b). The concept of comonotonicity in actuarial science and finance: applications. Insurance: Mathematics and Economics 31, 3-33.

[16] Doornik, J.A. (1996). OX. An Object - Oriented Matrix Programming Language. London: International Thompson Business Press.

[17] Embrechts, P., McNeil, A. and Strauman, D. (2002). Correlation and dependence in risk management: properties and pitfalls. In Risk Management: Value at Risk and Beyond, ed. by M. Dempster and H.K. Moffatt, 176-223.

[18] Frey,R. and McNeil, A. (2001). Modelling dependent defaults. Preprint, ZTH, Zürich.

[19] Frey, R., McNeil, A. and Nyfler, M. (2001). Copulas and credit models, Risk, October 2001, 111-114. 
[20] Gerber, H.U. (1982). Risk theory in the linear model. Insurance: Mathematics and Economics 1, 177-184.

[21] Goovaerts, M. and Dhaene, J. (1996). The compound Poisson approximation for a portfolio of dependent risks. Insurance: Mathematics and Economics 18, $81-85$.

[22] Goovaerts, M., Dhaene, J. and De Schepper, A. (2000). Stochastic upper bounds for present value functions. Journal of Risk and Insurance 67, 1-14.

[23] Hu, T. and Wu, Z. (1999). On dependence of risk and stop-loss premiums. Insurance: Mathematics and Economics 24, 323-332.

[24] Jacobs, P.A. and Lewis, P.A.W. (1978). Discrete time series generated by mixtures II: Asymptotic properties. J. R. Statist. Soc. B 40, 222-228.

[25] Johnson, L.N. and Kotz, S. (1997). Discrete Multivariate Distribution. New York: Wiley.

[26] Kaas, R., Dhaene, J., and Goovaerts, M. (2000). Upper and lower bounds for sums of random variables. Insurance: Mathematics and Economics 27, 151-168.

[27] Kaas, R., Goovaerts, M., Dhaene, J. and Denuit, M. (2001). Modern Actuarial Risk Theory, Kluwer Academic Publishers.

[28] Klimko, L. and Nelson, P. (1978). On conditional least squares estimation for stochastic processes. The Annals of Statistics 6, 629-642.

[29] Kolev, N. and Paiva, D. (2000). Correlated INAR(1) Process. In: Proc. Contributed Papers COMSTAT'2000, Utrecht, 337-342.

[30] Kolev, N. and Paiva, D. (2002a). Multinomial latent model for random sums. RT-MAE 2002-08, São Paulo. 
[31] Kolev, N. and Paiva, D. (2002b). Multinomial latent model for random sums. In: Proc. 2nd Conference in Actuarial Science 83 Finance on Samos, Karlovassi, 10-11.

[32] Luceño, A. (1995). A family of partially correlated Poisson models for overdispersion. Computational Statistics 83 Data Analysis 20, 511-520.

[33] Madsen, R. (1993). Generalized binomial distributions. Commmunications in Statistics: Theory and Methods 22, 3065-3086.

[34] MacDonald, I.L. and Zucchini, W. (1997). Hidden Markov and Other Models for Discrete-valued Time Series. Chapman and Hall, London.

[35] McKenzie, E. (1986). Autoregressive moving-average process with negativebinomial and geometric marginal distributions. Advances of Applied Probability 18, 679-705.

[36] McKenzie, E. (1988). Some ARMA models for dependent sequences of Poisson counts. Advances of Applied Probability 20, 822-835.

[37] Mirzai, B. (1999). On the rating of dependent risk. In: Proc. XXX ASTIN and 9th AFIR Colloquium, 95-102.

[38] Paiva, D. and Kolev, N. (2001a). Extended DAR(1) Process. In: Proc. 16th International Workshop of Statitical Modelling, 487-490.

[39] Paiva, D. and Kolev, N. (2001b). Correlação entre riscos dependentes e modelos de super-dispersão associados. In: Proc. $46^{a}$ Reunião Anual da RBRAS e $9^{a}$ SEAGRO. 459-462.

[40] Shumway, R.W. and Stoffer, D.S. (2000). Time Series Analysis and its applications. Berlin: Springer. 
[41] Simon S., Goovaerts, M. and Dhaene, J. (2000). An easy computable upper bound for the price of an arithmetic Asian option. Insurance: Mathematics and Economics 26, 175-184.

[42] Steutel, F.B. and van Harn, K. (1979). Discrete analogues of selfdecomposability and stability, The Annals of Probability 7, 893-899.

[43] Tallis, G.M. (1962). The use of generalized multinomial distribution in the estimation of correlation in discrete data. The Journal of Royal Statistical Society, Ser. B 24, 530-534.

[44] Vyncke, D., Goovaerts, M. and Dhaene, J. (2001). Convex upper and lower bounds for present value functions. Applied Stochastic Models in Business and Industry 17, 149-164.

[45] Wang, S. and Dhaene, J. (1998). Comonotocity, correlation order and premium principles. Insurance: Mathematics and Economics 22, 235-242.

[46] Wang, S. (1998). An actuarial index of the right-tail tisk. North American Actuarial Journal 2, 88-101.

[47] Winkelman, R. (2000). Econometric Analysis of count data, (3rd Edition). Berlin: Springer. 